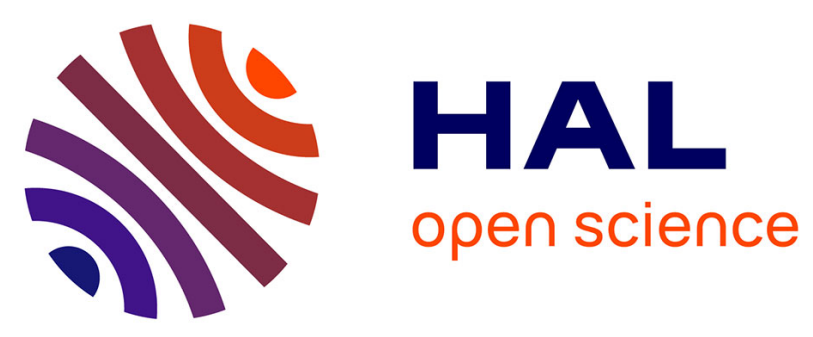

\title{
Une première campagne de prospection à Médamoud: méthodologie et résultats préliminaires (Mission Ifao/Paris-Sorbonne/Labex Resmed de Médamoud)
}

Félix Relats Montserrat, Julien Thiesson, Zulema Barahona Mendieta, Christelle Sanchez, Fayçal Rejiba, Roger Guerin

\section{To cite this version:}

Félix Relats Montserrat, Julien Thiesson, Zulema Barahona Mendieta, Christelle Sanchez, Fayçal Rejiba, et al.. Une première campagne de prospection à Médamoud: méthodologie et résultats préliminaires (Mission Ifao/Paris-Sorbonne/Labex Resmed de Médamoud). Bulletin de l'Institut Français d'Archéologie Orientale, 2016, pp.325-384. halshs-02087563

\section{HAL Id: halshs-02087563 \\ https://shs.hal.science/halshs-02087563}

Submitted on 15 Apr 2019

HAL is a multi-disciplinary open access archive for the deposit and dissemination of scientific research documents, whether they are published or not. The documents may come from teaching and research institutions in France or abroad, or from public or private research centers.
L'archive ouverte pluridisciplinaire HAL, est destinée au dépôt et à la diffusion de documents scientifiques de niveau recherche, publiés ou non, émanant des établissements d'enseignement et de recherche français ou étrangers, des laboratoires publics ou privés. 


\section{Bulletin de \\ L'INSTITUT FRANÇAIS D'ARCHÉOLOGIE ORIENTALE}

TOME 116

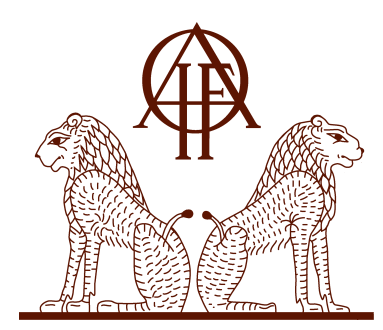

LE CAIRE - 2016 
Sommaire 


\section{Grenier Jean-Claude}

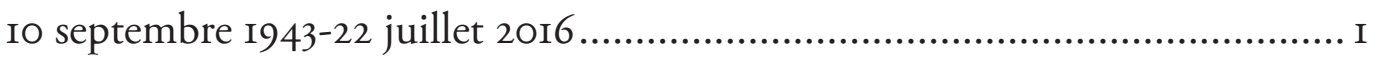

\section{Ashour Sobhi, Shueib Sayed}

A Roman Portrait-Head from Medinet Madi II

\section{Austin Anne, Gobeil Cédric}

Embodying the Divine:

A Tattooed Female Mummy from Deir el-Medina

\section{Chapon Linda}

Une possible représentation de l'arbre $j{ }^{2} d$

dans le temple de Millions d'Années de Thoutmosis III à Thèbes-Ouest

\section{Dorn Andreas, Polis Stéphane}

Nouveaux textes littéraires du scribe Amennakhte

(et autres ostraca relatifs au scribe de la Tombe)

\section{LAFONT Julie}

Consommation et proscription du miel en Égypte ancienne.

Quand bj.t devient bw.t..

\section{Mazé Christelle}

À la recherche des "classes moyennes». Les espaces de la différenciation sociale dans l'Égypte du III ${ }^{e}$ millénaire av. J.-C. .

\section{MeKawy Ouda Ahmed M.}

The Votive Stela of the "Overseer of the Singers of the King" Nfr-rnpt

(Egyptian Museum Cairo TR I4.6.24.17)

\section{Minart Marie-Anne}

Étude d'une cloison d'église de la fin du premier millénaire conservée au musée du Louvre. Monastère de Baouît, Moyenne Égypte.

\section{Minart Marie-Anne,}

\section{avec une contribution de BÉnAzeth Dominique}

Étude d'un vantail de la fin du premier millénaire conservé au musée du Louvre. Monastère de Baouît, Moyenne Égypte. 
Payraudeau Frédéric, Meffre Raphaële

Varia tanitica I

Vestiges royaux

Redon Bérangère, VANpeene Matthieu avec une annexe céramologique de Pesenti Mikaël

«La vigne a été inventée dans la ville égyptienne de Plinthine».

À propos de la découverte d'un fouloir saïte

à Kôm el-Nogous (Maréotide) 303

Relats Montserrat Félix, Thiesson Julien, Barahona-Mendieta Zulema, Sanchez Christelle, RéJiba Fayçal, Guérin Roger

Une première campagne de prospection à Médamoud: méthodologie et résultats préliminaires (Mission Ifao/Paris-Sorbonne/Labex Resmed de Médamoud) 325

\section{Töpfer Susanne}

„Aggressives Rosa“ - Zu einer Mumienauflage der spätptolemäisch-frührömischen Epoche aus Achmim (ÄMUL Inv.-Nr. 7810) 385 


\title{
Une première campagne de prospection à Médamoud: méthodologie et résultats préliminaires (Mission Ifao/ Paris-Sorbonne/Labex Resmed de Médamoud)
}

\author{
FÉLIX RELATS MONTSERRAT, JULIEN THIESSON, \\ ZULEMA BARAHONA-MENDIETA, \\ CHRISTELLE SANCHEZ, FAYÇAL RÉJIBA, ROGER GUÉRIN
}

$\mathrm{D}$ ES FOUILLES sur le site de Médamoud furent menées entre I925 et I940 par l'Ifao à la demande du musée du Louvre. Elles furent dirigées d'abord par F. Bisson de La Roque, puis par $\mathrm{Cl}$. Robichon, respectivement pensionnaire et architecte de l'Ifao. Elles se concentrèrent sur une petite surface du kôm antique, essentiellement le temple, ses fondations, l'espace ceinturé par le mur de clôture augustéen et le long du dromos jusqu'à la tribune (fig. I) I. Après une longue interruption, la mission a repris en $201 \mathrm{I}$ dans le cadre d'une coopération entre l'Ifao et l'université Paris-Sorbonne ${ }^{2}$. De 20II à 20I5, une nouvelle couverture photographique en couleur de l'ensemble du décor de la porte de Tibère a été réalisée par G. Pollin pour permettre à $\mathrm{D}$. Valbelle d'en réaliser le fac-similé des inscriptions, l'étude architecturale du monument étant confiée à E. Laroze ainsi que le travail de reconstitution des corniches en collaboration avec Fr. Burgos. L'édition des blocs de remplois est revenue à F. Relats Montserrat. À partir de 2OI2, ce dernier a repris l'étude de l'ensemble de la documentation disponible du site dans le cadre d'une thèse de doctorat ${ }^{3}$. Parallèlement, Z. Barahona-Mendieta a entrepris une étude de

1 Bisson De La Roque I926; Bisson de La Roque i927; Bisson de La Roque, Clere, Drioton i928; Bisson de la Roque, Clere i929; Bisson de La Roque i930; Bisson de La Roque I93I; Bisson de La RoQue 1933, Robichon, VARILle I939; Robichon, VARILle I94O.

2 http://www.ifao.egnet.net/archeologie/ medamoud. La mission est désormais dirigée par F. Relats Montserrat. Elle est constituée de D. Valbelle (égyptologue, université de la Sorbonne), d'E. Laroze (architecte - UMR 8167), G. Polin (photographe - Ifao), Fr. Burgos (tailleur de pierre - UMR 8167), Z. Barahona-Mendieta (Universitat Autonoma de Barcelona), Hassan Mohammad Ahmad (restaurateur - Ifao), Mustapha Ahmad Ali et Mohammad Mamdouh Mohammad Ali (inspecteurs du ministère des Antiquités). La partie géophysique était dirigée par J. Thiesson avec la participation de F. Réjiba,
R. Guérin, et Chr. Sanchez (UMR 7619 METIS). Ch. Bonnet et Y. Egels ont collaboré régulièrement aux activités sur le terrain. Nous remercions l'aide logistique du Centre franco-égyptien d'étude des temples de Karnak (CFEETK), ainsi que du ministère des Antiquités (Mohammad Abd-el Aziz, Amin Amar). 3 Université Paris-Sorbonne/ Labex Resmed/Fondation Thiers, voir Relats Montserrat 20i6b. 
la totalité de la documentation céramologique ${ }^{4}$. En raison du potentiel archéologique de ce site dont les anciennes fouilles ont déjà largement démontré la richesse et l'intérêt historique, la reprise d'un programme de prospections et de fouilles pouvait raisonnablement être envisagée. Grâce à un financement de la COMUE Sorbonne-Universités, la mission a pu établir un partenariat avec l'université Pierre-et-Marie Curie-Paris VI (UMR 76I9 METIS) afin de réaliser une prospection géomagnétique. Trois objectifs ont été établis:

I. Explorer le lien entre le Nil et le temple, notamment au niveau de la tribune.

2. Évaluer la pertinence des plans anciens issus des fouilles de F. Bisson de La Roque et de $\mathrm{Cl}$. Robichon, tout particulièrement à propos du tracé des murs de clôture et des fouilles, jamais publiées, de Cl. Robichon et A. Varille entre 1933 et I940.

3. Évaluer le potentiel archéologique de la zone du kôm, jamais fouillé.

Un quadrillage d'une maille de $20 \times 20 \mathrm{~m}^{2}$ a été créé afin de couvrir tout le kôm et permettre le repérage des différentes zones (fig. I). La dénomination de chacune d'entre elles donne le coin supérieur gauche (au nord-ouest) et le coin inférieur droit (au sud-est) de l'emprise prospectée. En plus de cette nomenclature, un nom d'usage (entre parenthèses dans le titre du paragraphe) a été donné pour faciliter le repérage global. En se reportant à la fig. I, on peut constater qu'un total Io zones a pu être complété, couvrant une surface d'à peu près I, 5 ha. Leur installation est sujette à la topographie du terrain, mais vise à répondre aux trois objectifs précédemment exposés. Pour évaluer la pertinence des plans anciens, le temple a été prospecté (avant-temple: QI4/PI5; cour d'Antonin le Pieux: Q13/Pi3), ainsi que son parvis où différents murs de clôture furent dégagés (Pıo/OII, Ni3). Nous avons également ajouté une petite zone à proximité de la tribune, où $\mathrm{Cl}$. Robichon découvrit en 1936 des massifs similaires à ceux encadrant la tribune de Tôd $\left(\mathrm{O}_{3}\right)^{5}$. Pour explorer le lien entre le Nil et le temple, nous avons loué un champ attenant à notre concession qui se trouve devant la tribune (RI/PI). Enfin, pour évaluer le potentiel archéologique de la zone du kôm, quatre zones plus ou moins distantes du temple ont été installées afin de livrer une vue suffisamment ample $\left(\mathrm{M}_{4} / \mathrm{K}_{9}, \mathrm{III}_{1} / \mathrm{H}_{12}, \mathrm{H}_{6} / \mathrm{G} 8, \mathrm{U}_{13} / \mathrm{V}_{13}\right)$.

Nous avons décidé de coupler cette recherche à une prospection céramologique menée par Z. Barahona-Mendieta. Elle se justifie par la richesse du site en matériel céramique, déjà signalée par le passé ${ }^{6}$, et notamment par la découverte de deux zones de fours à céramiques lors des fouilles de F. Bisson de La Roque ${ }^{7}$. Cependant, même si ces premiers résultats permettaient de classer Médamoud comme un des centres de production de Haute Égypte, aucune

4 Universitat autonoma de Barcelona, voir Barahona-Mendieta 2017.

5 Mentionnés par Pierrat et al. 1995, p. 450-463. Pour une publication complète: Relats Montserrat 2Oi6b.
6 P. Ballet avait déjà indiqué que Médamoud pouvait être considéré comme une zone de production de céramique à l'époque ptolémaïque (Ballet I99I, p. I39; Ballet 200I, p. II4).
7 Pour une actualisation céramologique des résultats, voir Barahona-Mendieta 2014. 
étude du matériel n'avait été conduite jusqu'à présent ${ }^{8}$. Par ailleurs, l'étude de la céramique de surface permet d'apporter des repères chronologiques et d'identifier les usages des zones de prospection géophysique.

Nous voulons présenter, dans les pages qui suivent, un premier aperçu des résultats obtenus, prélude à de prochaines campagnes. Nous avons décidé de présenter l'ensemble des résultats de la prospection céramologique afin d'offrir une vue globale de la richesse du site. La prospection géophysique n'est, quant à elle, détaillée que sur trois zones qui se focalisent sur les interrogations que nous avons précédemment exposées.

\begin{tabular}{|c|c|c|c|c|c|c|c|c|c|c|}
\hline \multirow[b]{2}{*}{ Méthodes } & \multicolumn{10}{|c|}{ Zones de prospection } \\
\hline & $\mathrm{V}_{\mathrm{I} 3} / \mathrm{U}_{13}$ & QI4/QI5 & Pio/OiI & $\mathrm{RI} / \mathrm{PI}$ & $\mathrm{M}_{4} / \mathrm{K}_{9}$ & $\mathrm{O}_{3}$ & Q13/Pr3 & HII/GI2 & D9/Cio & $\mathrm{N}_{3} 3$ \\
\hline CMD HCP & & $527 \mathrm{~m}^{2}$ & & & & $225 \mathrm{~m}^{2}$ & $350 \mathrm{~m}^{2}$ & & & \\
\hline CMD VCP & $760 \mathrm{~m}^{2}$ & & II82 $\mathrm{m}^{2}$ & $1050 \mathrm{~m}^{2}$ & $6090 \mathrm{~m}^{2}$ & & & $2100 \mathrm{~m}^{2}$ & $1020 \mathrm{~m}^{2}$ & \\
\hline G858 & $760 \mathrm{~m}^{2}$ & & $\mathrm{II} 82 \mathrm{~m}^{2}$ & & $5500 \mathrm{~m}^{2}$ & $225 \mathrm{~m}^{2}$ & $350 \mathrm{~m}^{2}$ & II $56 \mathrm{~m}^{2}$ & $1020 \mathrm{~m}^{2}$ & \\
\hline Radar & $760 \mathrm{~m}^{2}$ & & & & & 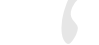 & & $1156 \mathrm{~m}^{2}$ & & \\
\hline $\mathrm{MS}_{2} \mathrm{D}$ & & & & & $5000 \mathrm{~m}^{2}$ & & & & & \\
\hline
\end{tabular}

TABL I. Surfaces prospectées avec les différents appareils.

8 Un des objectifs de la thèse de Z. Barahona-Mendieta, céramologue de la mission est d'aboutir à une synthèse des céramiques trouvées lors des premières fouilles du site
(Barahona-Mendieta 20i6). Un des objectifs de la mission est de poursuivre cette recherche dans les zones non encore fouillées. 


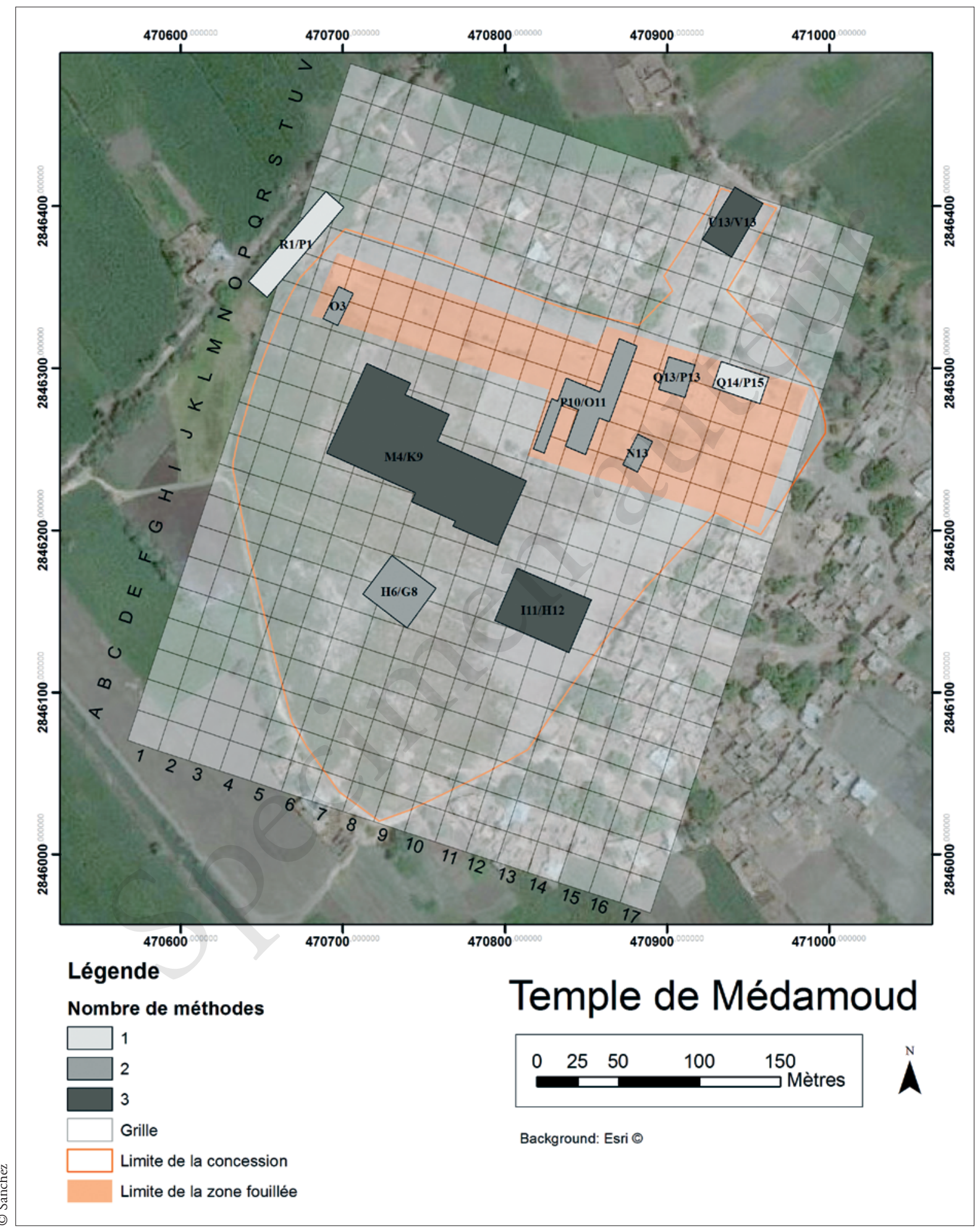

Fig. I. Zones prospectées avec quadrillage de la zone. 


\section{PROSPECTION GÉOPHYSIQUE : MÉTHODOLOGIE ET RÉSULTATS}

\section{Propriétés mesurées}

\section{Résistivité électrique ( $\rho$ ) ou conductivité électrique ( $\sigma$ )}

Cette propriété caractérise la difficulté $(\rho)$ ou la facilité $(\sigma)^{9}$ de déplacement de charges électriques (électron libre des métaux, ions des électrolytes) dans un milieu soumis à un champ électrique. Les principaux facteurs qui font varier $\sigma$ dans les sols sont la teneur en eau, sa minéralisation, la température et la teneur en argile. Dans le cadre du projet, la mesure de $\sigma$ avait pour objectif de différencier les zones pouvant correspondre à des structures de cuisson de céramique (en place ou détruites) plus résistantes électriquement. La mesure de $\sigma$ devait également permettre d'évaluer l'existence d'un canal en continuité du dromos par la présence d'un chemin préférentiel de l'humidité.

\section{Anomalie du champ magnétique et susceptibilité magnétique (к)}

Les contrastes d'aimantation du sous-sol engendrent des perturbations locales du champ magnétique terrestre. Ils sont soit induits (contraste de susceptibilité magnétique entre un matériau de remplissage superficiel et le terrain encaissant d'un fossé), soit rémanents (aimantation «mémorisée» en passant sous des températures de référence, dites de Curie, dans les briques cuites).

Dans le cadre du projet, la mesure de l'anomalie du champ magnétique terrestre permet particulièrement de reconnaitre les zones de fours à céramiques (avec présence de susceptibilité magnétique et d'aimantation rémanente) ainsi que d'appréhender les structures en brique crue (qui présentent des réponses marquées dans le cadre d'autres études ${ }^{\mathrm{I}}{ }^{\circ}$ ).

La susceptibilité magnétique est la propriété qui caractérise la capacité d'un matériau à acquérir une aimantation induite. Dans les sols, elle dépend principalement du contenu en minéraux magnétiques (argiles et oxydes de fer). Un changement d'utilisation du milieu, et en particulier l'occupation humaine, peut être caractérisé par une augmentation de la susceptibilité magnétique.

\section{Propriété apparente}

En géophysique appliquée, il est courant de représenter les cartes de mesures obtenues avec des dispositifs prenant en compte la réponse intégratrice d'un certain volume de sol en propriété dite apparente. Une propriété apparente est la valeur de la propriété considérée pour un terrain homogène qui donnerait la même réponse que celui mesuré effectivement. Les propriétés concernées dans la suite du texte (la conductivité/résistivité électrique et la susceptibilité magnétique) sont données en valeurs apparentes. 


\section{Remarques méthodologiques}

Les méthodes géophysiques sont principalement:

a. Non intrusives et non destructrices.

b. Indirectes, c'est-à-dire qu'elles ne donnent pas accès directement à une image du soussol, mais que celle-ci peut être proposée par une analyse.

c. Incertaines pour plusieurs raisons : des structures du sous-sol ou des activités anthropiques en surface peuvent induire un signal qui se superpose au signal cible rendant sa détection plus compliquée (bruits); plusieurs objets/structures différents peuvent sous certaines conditions provoquer des mesures identiques (phénomènes d'équivalence); et des objets/structures de nature proche peuvent sous certaines conditions provoquer des mesures très différentes (paradoxes).

d. Limitées par la profondeur d'investigation, définie comme étant la profondeur jusqu’à laquelle un objet/une structure peut se situer pour être détectée par les mesures géophysiques.

Étant donné le contexte archéologique de notre recherche, nous avons listé une série de vestiges susceptibles d'être présents sur le site. Le tabl. 2 liste les différentes propriétés géophysiques permettant a priori de les détecter.

\begin{tabular}{|l|l|}
\hline $\begin{array}{l}\text { Type de structure archéologique susceptible } \\
\text { d'être présente }\end{array}$ & Propriété mesurée \\
\hline mur en pierre & conductivité électrique (faible) \\
\hline \multirow{2}{*}{ briques cuites } & conductivité électrique (faible) \\
\cline { 2 - 2 } & susceptibilité magnétique (moyenne à forte) \\
\cline { 2 - 2 } & anomalie du champ magnétique (marquée) \\
\hline \multirow{2}{*}{ structures de cuisson (par exemple fours à céramique) } & conductivité électrique (faible) \\
\cline { 2 - 2 } & susceptibilité magnétique (moyenne à forte) \\
\cline { 2 - 2 } & anomalie du champ magnétique (marquée) \\
\hline \multirow{2}{*}{ canaux, fossés } & $\begin{array}{l}\text { conductivité électrique (forte) marquant le chemin préférentiel } \\
\text { d'humidité }\end{array}$ \\
\hline \multirow{2}{*}{ briques crues } & susceptibilité magnétique (faible) \\
\cline { 2 - 2 } & conductivité électrique (plus élevée que l'encaissant) \\
\hline \multirow{2}{*}{ tranchée remblayée } & susceptibilité magnétique (plus élevée que l'encaissant) \\
\cline { 2 - 2 } & anomalie du champ magnétique (marquée) \\
\hline
\end{tabular}

TABL. 2. Récapitulatif des propriétés mesurées.

\section{Appareils utilisés et échantillonnage}

La campagne a consisté en la réalisation de deux types de mesures:

I. La cartographie: les données sont acquises avec une géométrie fixée pour l'ensemble de la prospection. Les mesures sont effectuées suivant un maillage régulier dont chaque point peut être identifié à l'aide de ses coordonnées dans un repère orthonormé permettant ensuite de réaliser des cartes des variations apparentes des propriétés. 
2. La mesure sur structure décapée: les données sont acquises le long d'un profil avec une géométrie donnée. L'opération est répétée afin d'évaluer l'homogénéité et la répétabilité des valeurs mesurées.

\section{Cartographie}

Nous avons réalisé la carte de trois paramètres:

I. La conductivité électrique apparente a été mesurée avec un dispositif électromagnétique à faible nombre d'induction de type slingram, le CMD Mini Explorer de GF Instruments. L'appareil dispose d'une configuration coplanaire qui permet d'effectuer les mesures en mode dipôles magnétiques verticaux ou DMV (ou configuration bobines horizontales coplanaires ou HCP) et en mode dipôles magnétiques horizontaux ou DMH (ou configuration bobines verticales coplanaires ou VCP). Il permet la mesure simultanée de trois écartements entre émettrice et réceptrice (0,32 m, 0,7I m et I,I $8 \mathrm{~m})$. En mode DMV, les profondeurs sont, pour la mesure de conductivité électrique, de l'ordre de I,5 fois l'écartement, avec un poids important pour les objets/structures situés à 0,4 fois l'écartement. En mode DMH, les profondeurs sont, pour la conductivité électrique, de l'ordre de 0,8 fois l'écartement, avec un poids important pour les objets/structures situés en surface. L'acquisition s'est faite par profils espacés de $0,5 \mathrm{~m}$ avec un point enregistré toutes les $0,3 \mathrm{~s}$. La maille restituée est de $0,25 \times 0,25 \mathrm{~m}^{2}$ après interpolation.

2. L'anomalie du champ magnétique et son pseudo-gradient ont été mesurés par un gradiomètre G858 de Geometrics ${ }^{I I}$. La mesure s'effectue à pas de temps régulier (O,I s) le long de profils espacés de $0,5 \mathrm{~m}$. La maille restituée est de $0,25 \times 0,25 \mathrm{~m}^{2}$ après interpolation. Nous ne pouvons pas associer de profondeur d'investigation à cette méthode.

3. Enfin, la susceptibilité magnétique apparente a été mesurée grâce au CMD Mini Explorer de GF Instruments, simultanément à la conductivité électrique, uniquement pour le mode DMH. Les profondeurs atteintes sont de l'ordre de 0,7 fois l'écartement et la maille restituée est de $0,25 \times 0,25 \mathrm{~m}^{2}$ après interpolation.

Les résultats sont accompagnés d'une carte d'interprétation, obtenue suivant le protocole décrit en annexe. Elle sert à mettre en valeur les anomalies les plus intéressantes, par exemple le découpage en zones de fortes et de faibles valeurs du paramètre mesuré.

\section{Mesure sur structure décapée}

- La susceptibilité magnétique apparente superficielle a été mesurée grâce au susceptibilimètre $\mathrm{MS}_{2}$ de Bartington Ltd équipé du capteur $\mathrm{MS}_{2} \mathrm{D}$, une boucle de $0,18 \mathrm{~m}$ de diamètre. Les mesures ont été effectuées suivant un maillage de $0,2 \times 0,2 \mathrm{~m}^{2}$.

- La résistivité électrique a été mesurée à l'aide d'un prototype de résistivimètre léger RMCA5. La géométrie utilisée est celle d'un dispositif wenner alpha avec un écartement de 0,2 m entre les électrodes consécutives et un maillage de $0,2 \times 0,2 \mathrm{~m}^{2}$

11 Les capteurs à vapeur de césium étaient disposés en mode gradient vertical avec un espacement de $0,65 \mathrm{~m}$ et une hauteur au-dessus du sol du capteur du bas autour de 0,4 $\mathrm{m}$. 


\section{Résultats $^{12}$}

Chacune des zones prospectées a donné des résultats, présageant de la présence de vestiges, mais nous avons renoncé à en présenter la totalité. Nous centrons notre propos sur les trois zones qui peuvent être interprétées archéologiquement et qui permettent de répondre aux interrogations précédemment exposées. En premier lieu nous présenterons la prospection portant sur la relation entre le temple et le $\mathrm{Nil}(\mathrm{RI} / \mathrm{PI})$, puis celle permettant d'évaluer et de recaler les plans des anciennes fouilles (Pro/OII) et enfin celle livrant des informations sur l'occupation du kôm $\left(\mathrm{M}_{4} / \mathrm{K}_{9}\right)$. Les résultats des autres zones serviront de base à l'ouverture de sondages lors des prochaines saisons.

\section{Zone RI/PI (canal)}

La zone est hors de l'enceinte de notre concession et mesure $15 \times 70 \mathrm{~m}^{2}$. Elle a été prospectée avec l'accord du propriétaire, profitant de l'absence de culture sur le terrain. Nous présentons les cartes de susceptibilité magnétique et de conductivité électrique apparentes acquises avec le CMD Mini Explorer en configuration VCP.

\section{- Susceptibilité magnétique CMD}

Les cartes de susceptibilité magnétique (fig. 2) font apparaitre une tendance est-ouest allant des valeurs les plus faibles (couleur froide) aux plus fortes (couleur chaude). Même si cette tendance est la même quel que soit l'écartement, la voie 3 (I,I8 $\mathrm{m}$ de profondeur) semble faire apparaître des anomalies liées à des structures qui ne sont pas aussi visibles sur les deux autres voies.

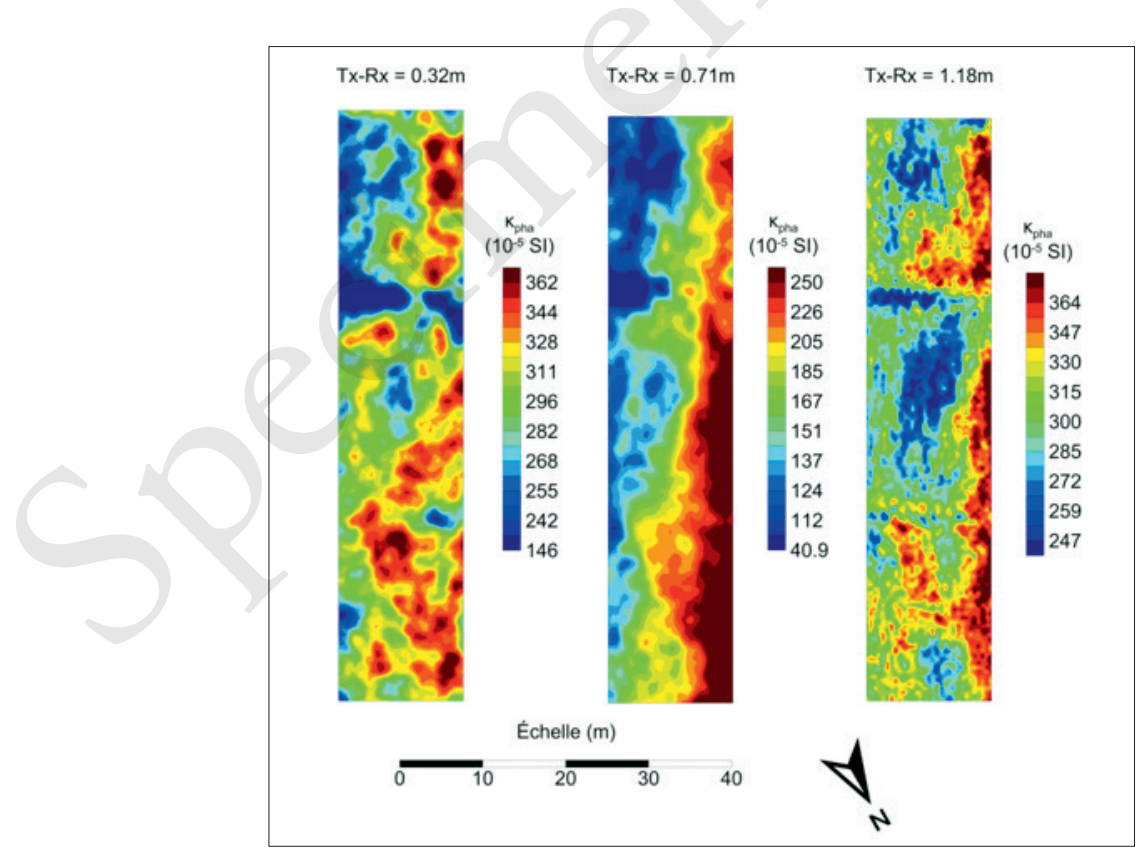

Fig. 2. Carte de susceptibilité magnétique obtenue sur la zone $\mathrm{R}_{\mathrm{I}} / \mathrm{PI}$.

12 Sauf mention contraire, les différentes cartes sont présentées avec une échelle de couleur adaptée, afin de faire au mieux ressortir la dynamique propre à chaque mesure. 
- Conductivité électrique CMD

Les cartes de conductivité électrique de la zone (fig. 3) montrent une zone de fortes valeurs au centre de la parcelle. Quelques anomalies de plus forte conductivité apparente sont également visibles dans le tiers nord (en bas des cartes) et dans le tiers sud (en haut des cartes). Elles ne présentent pas de rapport de direction avec l'anomalie centrale.

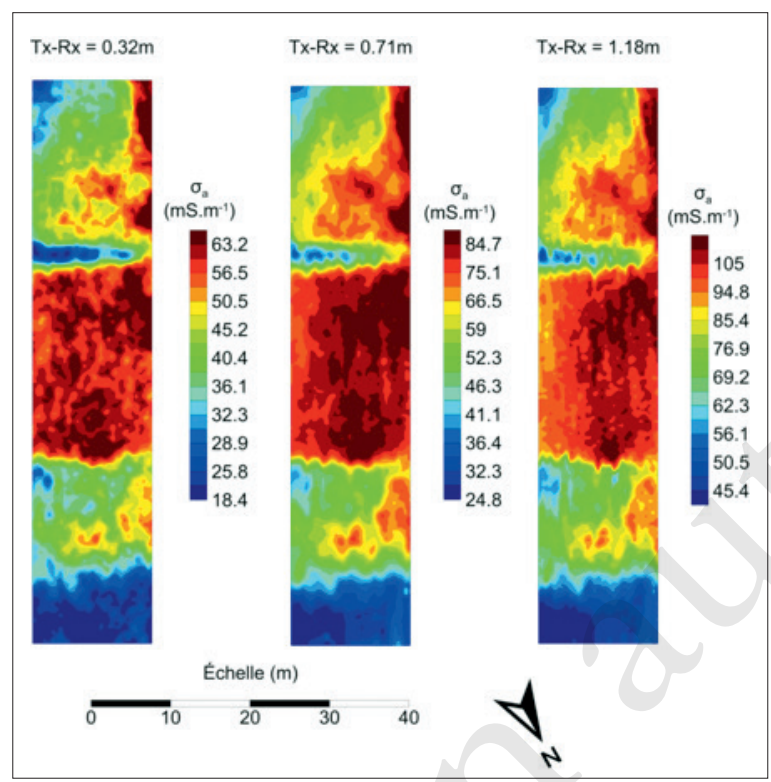

FIG. 3. Carte de conductivité électrique obtenue sur la zone RI/PI.

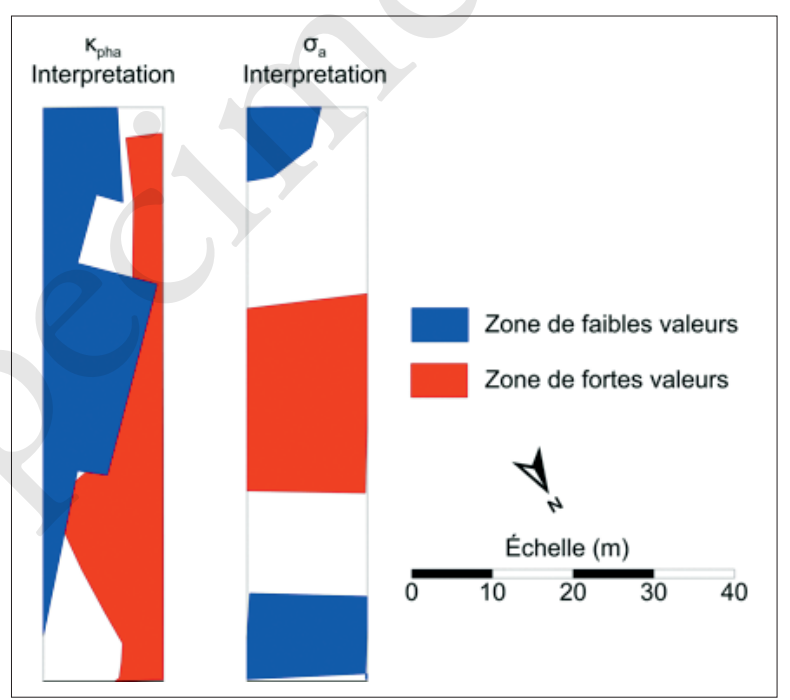

Fig. 4. Carte d'interprétation de RI/Pr.

D'après les cartes d'interprétation (fig. 4), la partie centrale de la parcelle semble vraiment plus conductrice. Cette zone apparaît également comme une zone de faible susceptibilité s'étendant quasiment sur toute la largeur. Une partie de ces anomalies semblent s'orienter suivant une direction nord-ouest - sud-est. 


\section{Zone Pio/OII (poterne)}

La zone de la poterne a été prospectée pour essayer de confirmer que les vestiges dégagés par les fouilles anciennes sont encore présents malgré l'absence de signes visibles en surface. Il faut être conscient que plusieurs décrochements topographiques sont présents sur la surface prospectée qui obligent à une grande prudence dans l'interprétation des anomalies et qui expliquent la forme de la zone prospectée. Il faut également signaler qu'au nord de la prospection se trouve le dallage en pierre reliant la porte de Tibère aux kiosques. Cela entraîne une faible réponse en susceptibilité magnétique et en conductivité électrique.

- Susceptibilité magnétique CMD

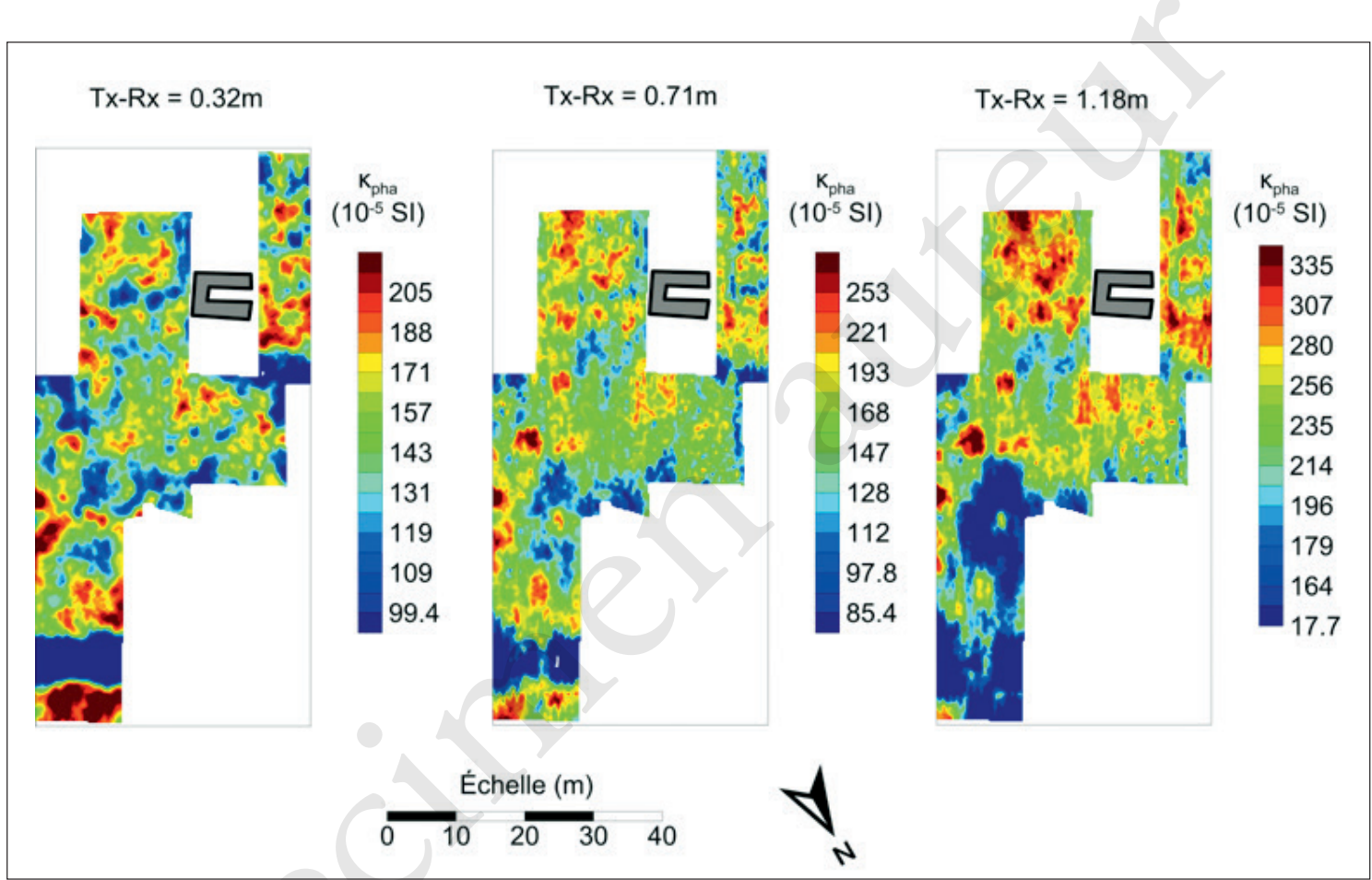

Fig. 5. Carte de la susceptibilité magnétique sur la zone Pıo/OıI.

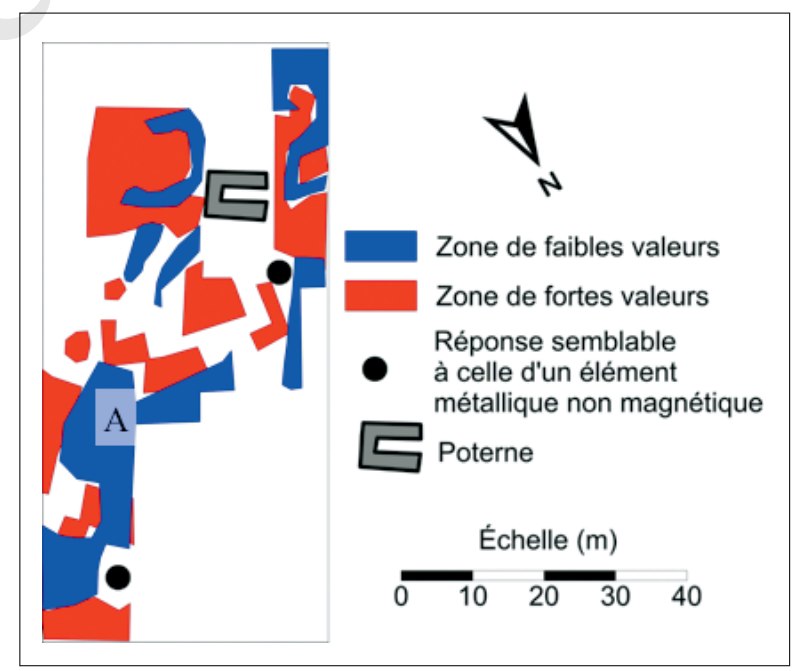

Fig. 6. Interprétation de la zone Pio/Oıı. 
Les cartes de susceptibilité magnétique (fig. 5) permettent de distinguer un certain nombre d'anomalies. D'après l'interprétation (fig. 6), plusieurs orientations ressortent. Tout d'abord, une orientation est-ouest qui semble se retrouver sur des anomalies positives et négatives (notamment au sud, autour de la poterne). Ensuite, il apparaît une longue anomalie négative (lettre A) orientée sud-ouest - nord-est qui, bien que coupée par des anomalies positives, semble se distinguer sous la forme de deux anomalies parallèles dans sa partie sud.

- Conductivité électrique CMD

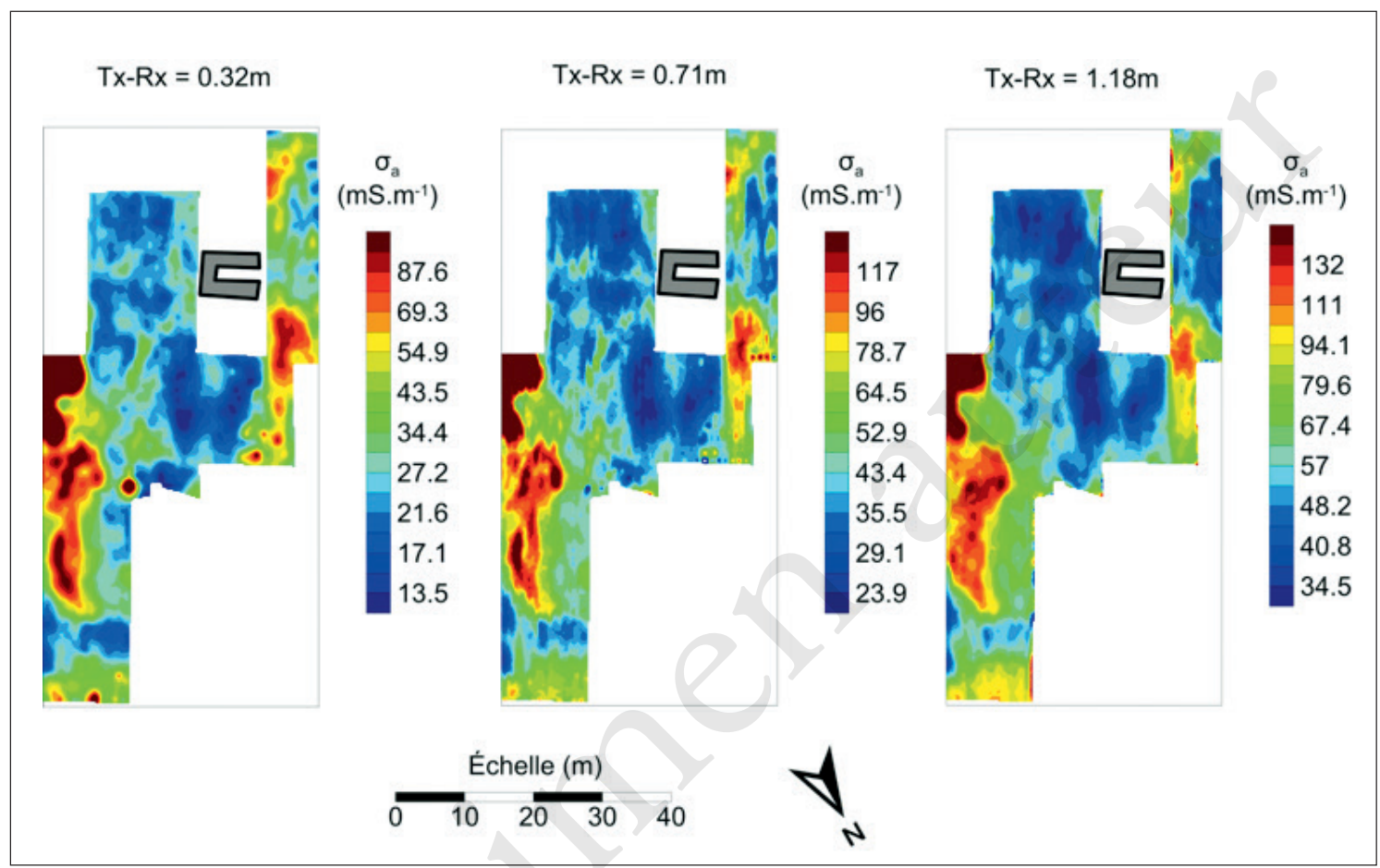

Fig. 7. Cartes de conductivité électrique de la zone.

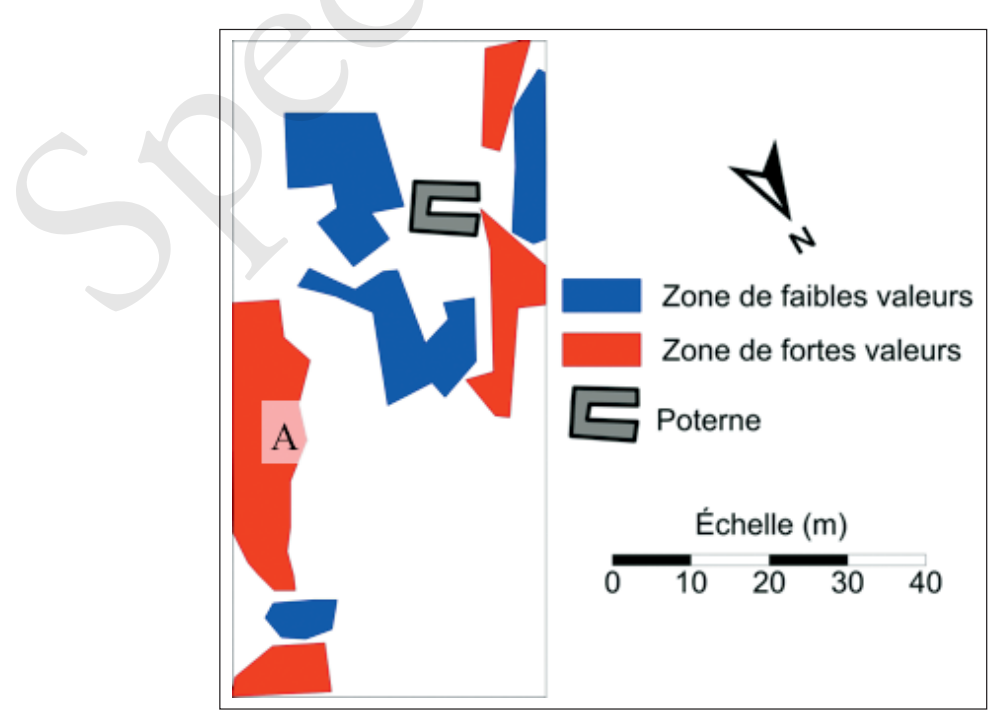

Fig. 8. Carte d'interprétation de la conductivité. 
Les cartes de conductivité électrique (fig. 7) montrent deux zones de valeurs plus élevées (à l'est et à l'ouest) séparées par une zone intermédiaire (partie centrale). Sur la carte d'interprétation (fig. 8), les fortes anomalies les plus à l'est de la prospection semblent dessiner une forme d'arc de cercle (lettre A). Il n'y a pas d'orientation préférentielle qui se démarque sur cette carte

\section{- Anomalie du champ magnétique G858}

Les résultats présentés ne sont que ceux du capteur du bas, le capteur du haut ayant dysfonctionné.

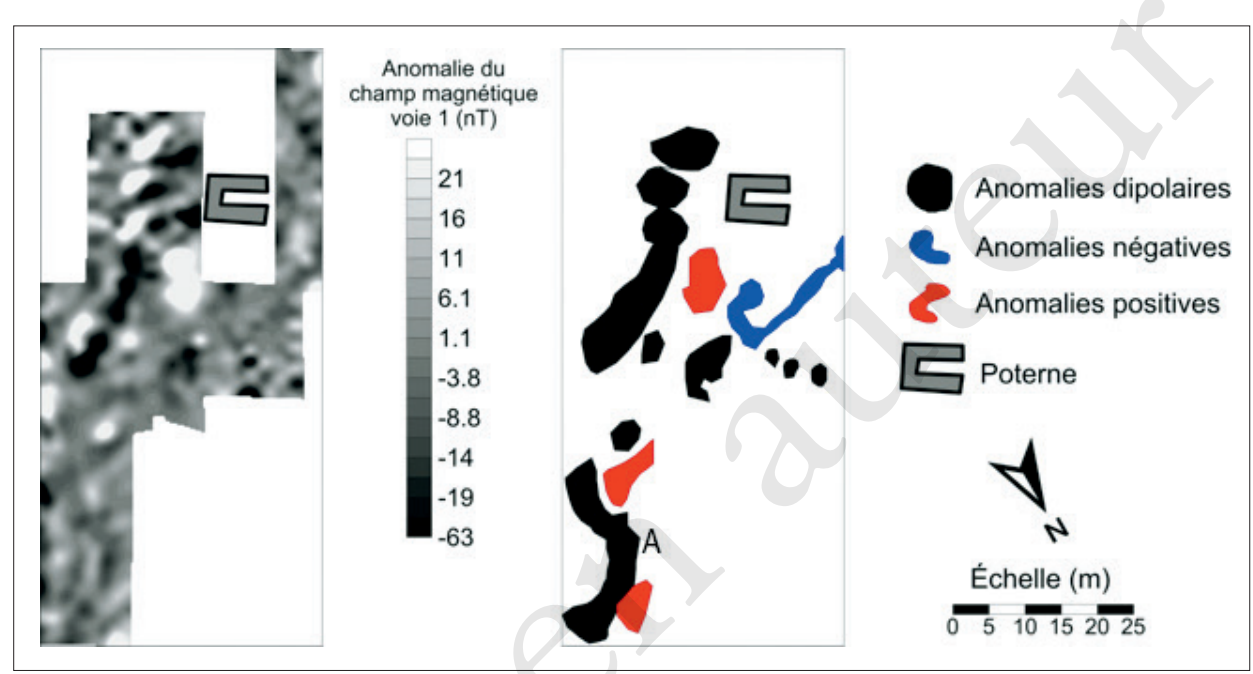

Fig. 9. Carte d'anomalie magnétique du capteur du bas (gauche) et interprétation (droite) sur la zone Pio/Oir.

La carte d'anomalie magnétique (fig. 9) laisse apparaître plusieurs groupes d'anomalies intéressants. Tout d'abord le groupe d'anomalies dipolaires dans la partie sud qui semble former un alignement d'orientation nord-est - sud-ouest. Le groupe A présente une forme intéressante en arc de cercle qui semble répondre aux deux anomalies positives visiblement est-ouest. Enfin, une anomalie négative semble également suivre une orientation est-ouest (partie est de la prospection).

\section{Zone $M_{4} / K 9$ (grande zone)}

Cette zone a été prospectée afin d'évaluer le potentiel archéologique hors de l'emprise du temple et des fouilles anciennes. C'est la plus grande surface prospectée d'un seul tenant. 
- Susceptibilité magnétique CMD

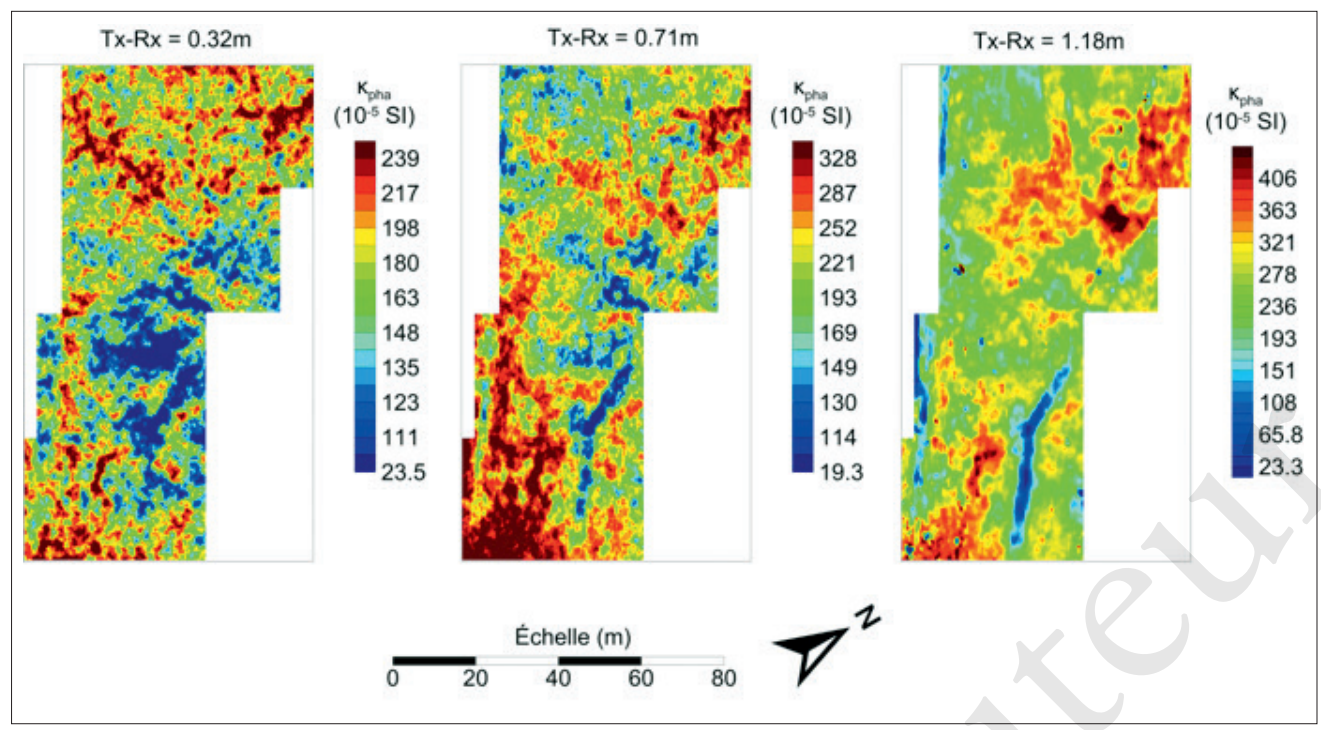

Fig. Io. Carte de susceptibilité magnétique obtenue sur la zone M4/K9.

Les cartes de susceptibilité magnétique (fig. Io) laissent apparaître un grand nombre d'anomalies qui peuvent être associées à des structures enfouies. Il semble que la structuration change avec les écartements (et donc la profondeur).

En effet, sur la carte interprétée (fig. II), on peut observer que l'extension des zones d'anomalies est plus importante aux profondeurs les plus faibles. Les anomalies les plus faibles dessinent des ensembles cohérents en termes d'orientation (nord-ouest - sud-est et perpendiculaire). Une des structures semble en particulier se prolonger sur la partie est de la carte en ayant une forme allongée, interrompue avant un léger changement de direction par des structures perpendiculaires. Il est à noter que les structures avec fortes valeurs sont, quant à elles, plutôt orientées nord-est.

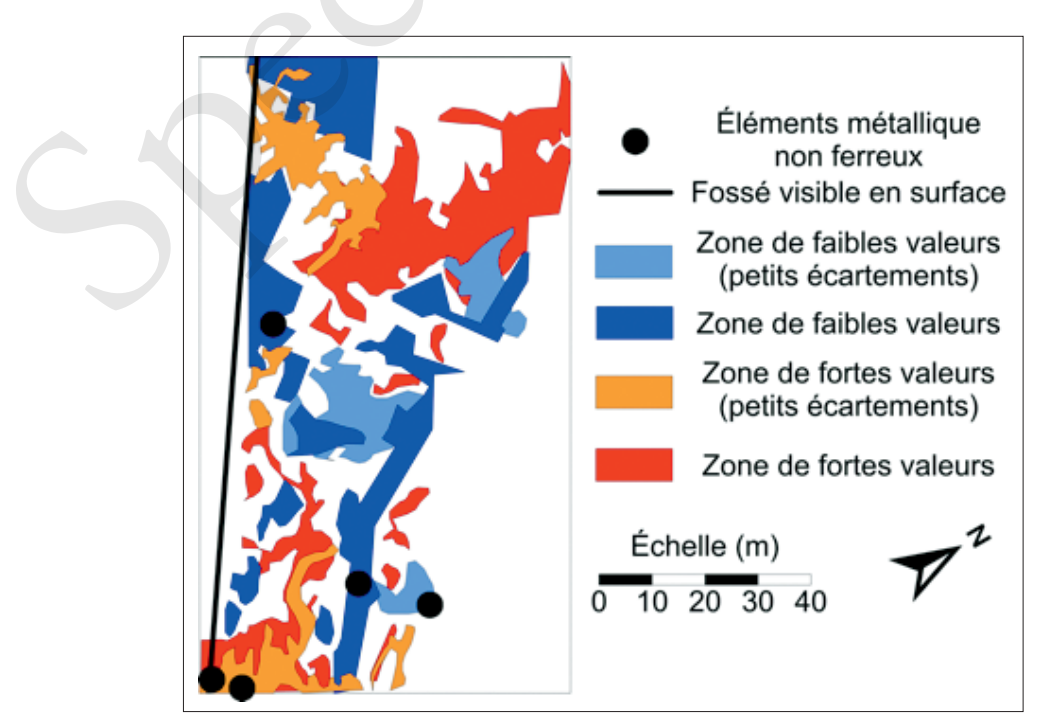

FIG. II. Interprétation de la susceptibilité magnétique de M4/K9. 
- Conductivité électrique CMD

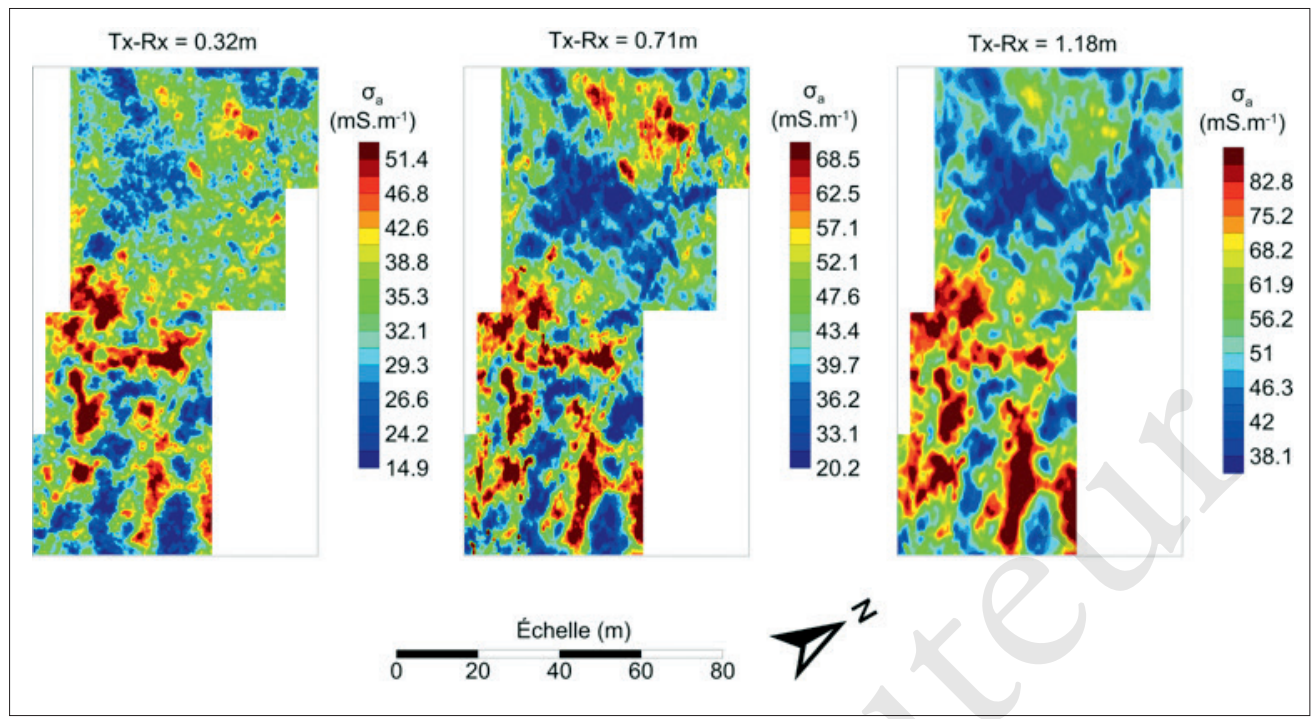

FIG. I2: Carte de conductivité électrique obtenue sur la zone $\mathrm{M}_{4} / \mathrm{K}_{9}$.

Les cartes de conductivité électrique (fig. I2, I3) montrent un certain nombre d'anomalies dont certaines peuvent être associées à des structures sous-jacentes. Il semble qu'une partie des grands ensembles peu conducteurs deviennent plus marqués et étendus avec la profondeur.

Dans la partie la plus au sud et à l'est de la prospection, les anomalies apparaissent comme un réseau de direction nord-ouest - sud-est avec des retours perpendiculaires tant de fortes ou de faibles valeurs. Dans la partie la plus au nord et à l'ouest, les orientations ne sont plus les mêmes et apparaissent plutôt nord-sud.

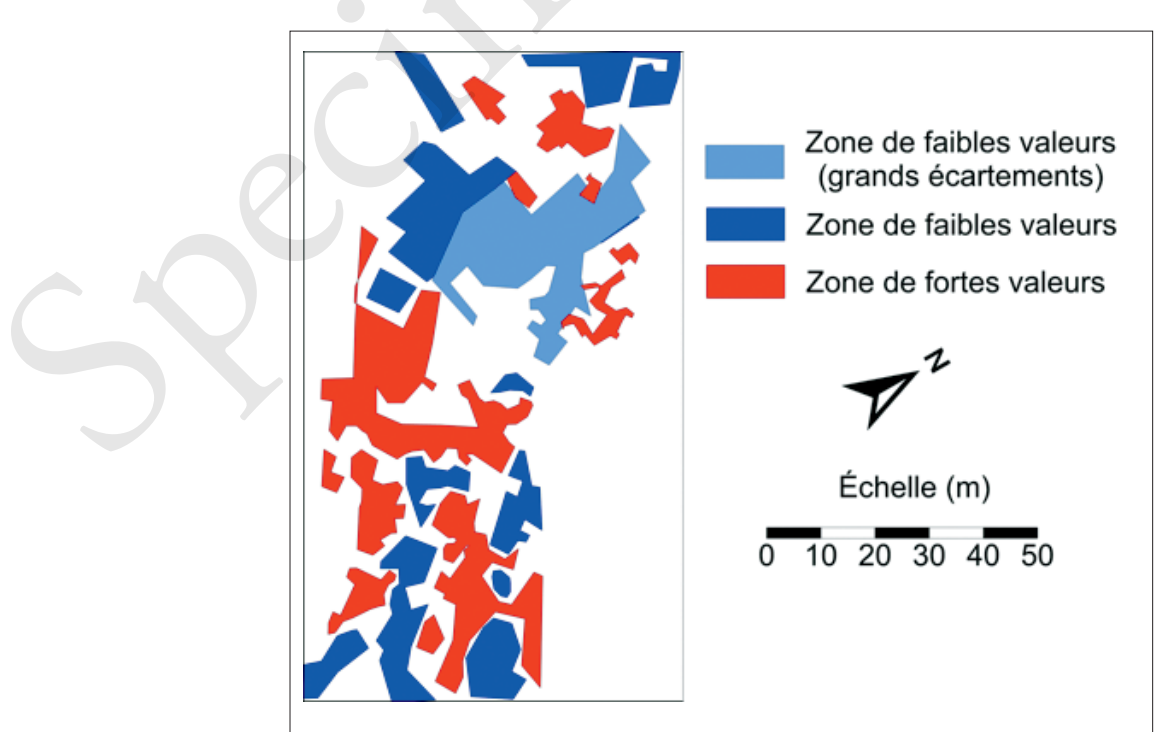

FIG. I3. Carte de la conductivité électrique interprétée sur la zone $\mathrm{M}_{4} / \mathrm{K}_{9}$. 
- Anomalie du champ magnétique G858

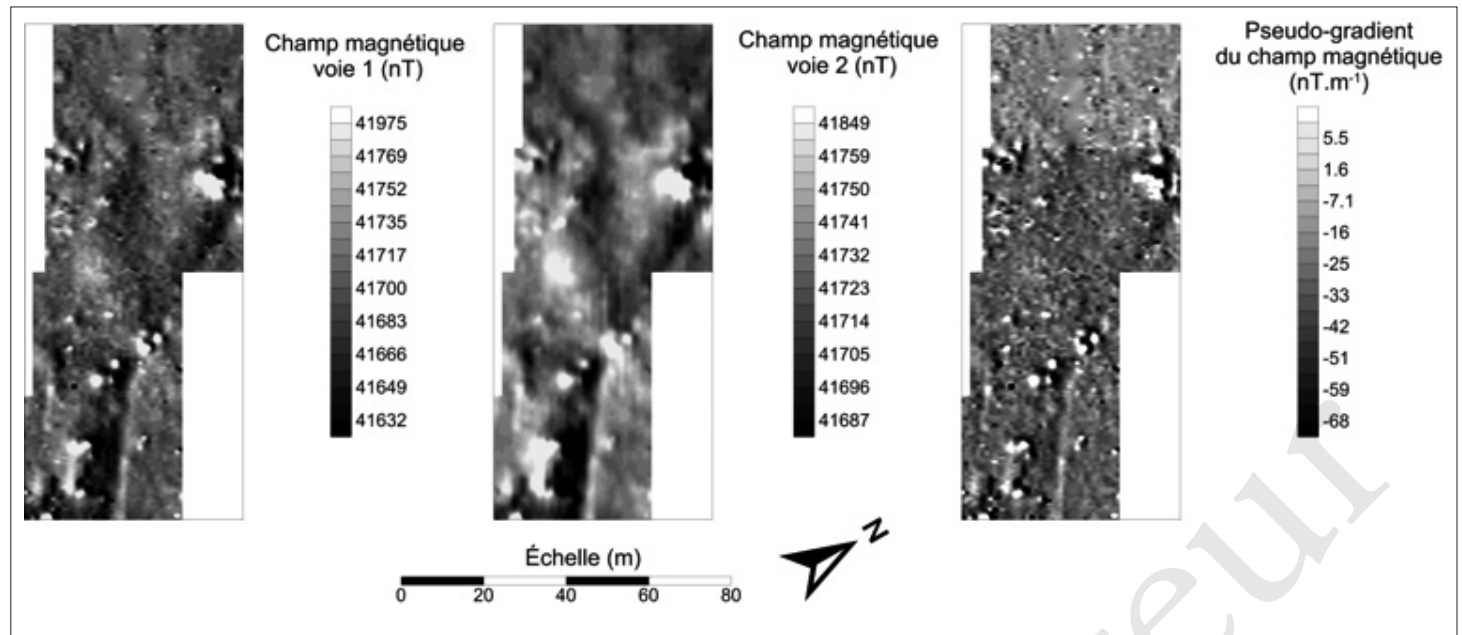

FIG. I4. Carte de champ total et de pseudo-gradient obtenue sur $\mathrm{M}_{4} / \mathrm{K}_{9}$.

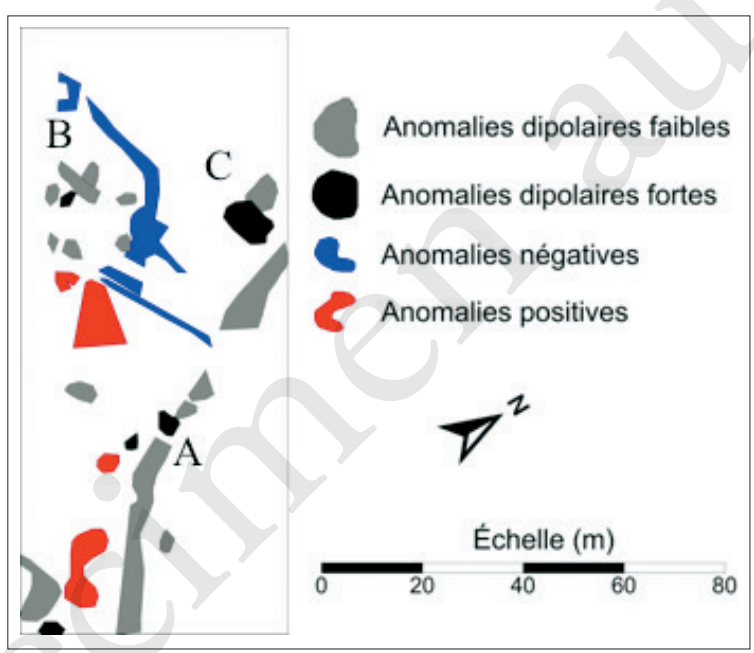

FIG. 15. Carte d'interprétation des anomalies d'intérêt sur $\mathrm{M}_{4} / \mathrm{K}_{9}$.

Les cartes d'anomalies et de pseudo-gradient du champ magnétique (fig. I4) montrent une grande richesse d'anomalies. Les anomalies les plus notables (fig. I5) présentent un allongement dont les directions sont est-ouest, voire nord-est - sud-est pour les anomalies négatives. Les anomalies dipolaires allongées ont quant à elles des directions nord-ouest - sud-est avec un changement dans la partie sud. Enfin, on peut voir plusieurs "grappes » d'anomalies dipolaires concentrées (A, B et C).

Les résultats de la prospection géophysique sont donc d'une très grande richesse, mais ne peuvent pas être interprétés seuls. Nous pouvons certes distinguer les directions préférentielles et les formes de certaines anomalies qui, dans le cas présent, ne présentent pas une organisation spatiale évidente. Cela témoigne de l'abondance de vestiges et de leur accumulation stratigraphique qui empêche d'en présenter un plan. Pour aboutir à une interprétation globale, nous devons donc prendre en compte les résultats d'autres méthodes. 


\section{LA PROSPECTION CÉRAMOLOGIQUE}

La prospection céramique s'est essentiellement déroulée en 2015 , mais nous intégrons également les résultats de la campagne précédente, d'avril et octobre $20{ }^{2}{ }^{\mathrm{I}}$. Elle avait pour objectifs, d'une part, de compléter l'étude géophysique en apportant des repères chronologiques et une identification des usages de chacun des secteurs prospectés et, d'autre part, de présenter un premier aperçu de la nature des dépôts céramiques sur le kôm. Même si nous n’avons pas pu réaliser une prospection globale du site, les zones choisies présentent déjà de fortes disparités. Nous avons donc limité nos recherches aux secteurs où la prospection géophysique a pu être réalisée même si, en raison des caractéristiques du terrain ainsi que de la nature des concentrations de tessons, les secteurs de prospection céramique sont plus larges. Pour pouvoir donner un état global de la documentation, nous présentons ici l'ensemble des secteurs.

Au sein de ceux-ci, les céramiques les plus significatives du point de vue chronologique ont été identifiées. Dans un deuxième temps, nous avons estimé la densité et l'accumulation des différentes catégories typologiques, afin de connaître l'usage et la nature des secteurs. C'est pourquoi nous avons non seulement recueilli les tessons avec des formes datables, mais nous avons également enregistré leur récurrence et les caractéristiques propres du terrain, sans pour autant effectuer des prélèvements systématiques à une distance fixe. Dans la mesure où Médamoud devait être un centre important de production céramique, nous avons identifié tous les indices pour reconnaître les ateliers de potiers tels que les scories, les déchets de cuisson et les briques vitrifiées ${ }^{\mathrm{I}}$. Pour les zones les plus significatives, nous avons dressé un schéma localisant les principales accumulations en incluant les numéros des céramiques pour localiser leur provenance.

Avant de présenter les résultats, nous pouvons d'ores et déjà caractériser la nature du terrain d'un point de vue global. Très vite nous est apparue la grande quantité de céramiques réparties sur toute la zone archéologique, si bien que plusieurs milliers de tessons sont visibles en surface $^{\mathrm{Is}}$. D'autre part, sont également répandus sur tous les secteurs un très grand nombre de scories, de tessons déformés (voir fig. I7B), surcuits ou vitrifiés - restes évidents de la production de céramiques - et de briques vitrifiées sur une de leurs faces - qui provenaient des parois des fours eux-mêmes. Enfin, $90 \%$ des tessons visibles à la surface sont faits en pâtes de type calcaire et seul Io \% en pâtes alluviales. Ce premier constat permet de supposer que la production locale était faite à base de pâtes calcaire. En ce sens, les tessons de pâte Marl A4 var. 2 de Basse Époque et d'époque ptolémaïque sont surreprésentés dans toutes les zones, ce qui confirme le caractère industriel de cette production (fig. I6B) ${ }^{16}$. En ce qui concerne les pâtes

\footnotetext{
13 Sur ces prospections voir Barahona-MENDIETA 2017 ; Barahona-Mendieta, Relats Montserrat 20I7. La prospection a été réalisée par Z. Barahona-Mendieta, avec la collaboration en 2014 de Chr. Sanchez.

14 Les espaces de production céramique ont été reconnus d'après les
}

critères suivants: abondance des fragments de céramique; concentration de formes identiques, des caractéristiques morphologiques et des pâtes; mais surtout présence de déchets de coction, scories, fragments surcuits de couleur verdâtre, déformés et vitrifiés et restes de fours (BALlet et al. I99I, p. I3O-I34).
15 Certaines zones, non prospectées cette année, ont leur sol recouvert par deux ou trois couches de tessons, tout particulièrement au sud-ouest du kôm (zones $\mathrm{F}_{5}, \mathrm{E}_{5}, \mathrm{E} 6$ ).

16 Barahona-Mendieta 2017. 
alluviales, la grande majorité correspond à des parois d'amphores byzantines du type $L R 7$ et à des conteneurs présentant des caractéristiques similaires, mais dont aucune forme n'a été bien conservée (petits tessons et plusieurs pivots). Dans l'ensemble des zones, le même constat a pu être établi et, autour du dromos seulement, la quantité de céramique byzantine est plus élevée.

Nous allons désormais présenter un catalogue raisonné des tessons recueillis les plus représentatifs. Celui-ci vise à être le plus exhaustif possible, la majorité des pièces trouvées ont ainsi été retenues. Notre présentation se fera secteur après secteur, en identifiant les types de céramiques: nous en offrirons un rapide commentaire avant d'en préciser la localisation originelle ${ }^{\mathrm{I}}$.

\section{Zone nord}

\section{Secteurs $U_{13} / V_{14}$}

La prospection a été étendue légèrement vers le nord, en couvrant également le petit terrain au nord de VI3. La quantité de céramique en surface est assez réduite par rapport aux autres secteurs du kôm. La plupart des tessons collectés appartiennent à l'époque byzantine. De manière résiduelle apparaissent également plusieurs tessons d'amphores romaines ainsi que quelques fragments en pâte $\mathrm{Marl} A_{4}$ var. 2 de Basse Époque. Il faut noter la présence d'un grand tesson de African Red Slip Ware importé qui correspond à la forme Hayes 82, daté du milieu du ve/début du vi ${ }^{\mathrm{e}}{ }^{\mathrm{e}}{ }^{\mathrm{I} 8}$ (pl. I5. Medi5-Ui3.I), ainsi que celle de différents fragments de jattes en pâte calcaire s'étalant du $\mathrm{I}^{\mathrm{er}}$ apr. J.-C. jusqu'au début de l'époque byzantine. Ces derniers furent probablement produits à Médamoud étant donné que des déchets de coction ont été trouvés sous la forme de fragments vitrifiés par exposition à de hautes températures (pl. I4. Medi5-Ui3.I2 ${ }^{19}$ et Medi5-A.3 ${ }^{20}$ ). Il faut également faire mention d'une lampe complète moulée en pâte d'Assouan datée des v/vi s. ${ }^{2 \mathrm{I}}$ (pl. I5. Medi5-Ui3.4).

\section{La zone du temple}

\section{Secteurs Pg/MII et $\mathrm{OI}_{12} / \mathrm{MI}_{3}$ (poterne)}

La grande majorité de la céramique de surface observée dans cette zone correspond à une fourchette chronologique allant de la Deuxième Période intermédiaire à la XVIII ${ }^{\mathrm{e}}$ dynastie. Il est probable que ces céramiques proviennent d'anciens sondages réalisés par F. Bisson de La Roque (en 1930) et par Robichon et Varille (entre 1933 et 1940) ${ }^{22}$. En effet, le premier avait déjà fouillé le secteur PII-OII-NII et avait trouvé une série de fours ${ }^{23}$ dont la céramique a

17 Afin de ne pas multiplier les dessins de pièces similaires, une seule figure a été dessinée par type identifié.

18 BONIFAY 2004, fig. 89, typi Sigille 27.

19 Tomber 2006, fig. I.38, 8/509.
20 Gempeller i992, Abb. 70, Tippo 604-5; LaufFraY 1995, fig. 42, 348; Pierrat 1996, fig. I2, 6I.

21 Bailey i988, pl. 53, fig. q2239.

22 Pour un état de la question céramologique, voir Barahona-Mendieta 2014.
Pour une chronologie des secteurs fouillés par Robichon et Varille, voir ReLATS Montserrat 2OI6b.

23 La présence de ces fours a motivé la prospection géomagnétique du secteur: cf. infra. 
déjà été étudiée par Z. Barahona-Mendieta ${ }^{24}$. Or, il protégea les vestiges en les recouvrant avec le remblai qu'il avait préalablement dégagé du même emplacement. Étant donné que les pâtes des céramiques de surface sont identiques à celles qui furent découvertes (Marl $A 2$, Marl $A_{4}$ var. I et $\operatorname{Marl} B$ ), qu'elles présentent une chronologie similaire, et que nous avons aussi trouvé des scories, des tessons déformés et surcuits, nous pensons qu'il faut les considérer comme le produit des anciennes fouilles. Il est ainsi possible que lors du remblayage de la zone, des céramiques aient remonté à la surface. Cependant, trois fragments pourraient remonter à une époque antérieure à celle des découvertes de F. Bisson de La Roque, à savoir le Moyen Empire. Deux explications peuvent être avancées: soit F. Bisson de La Roque atteignit, sans s'en rendre compte, des niveaux antérieurs; soit, plus vraisemblablement, ils proviendraient de sondages effectués par Ch. Robichon et A. Varille dans ces secteurs quand ils cherchèrent le temple de Sésostris III et le Temple Primitif ${ }^{25}$.

De ces pièces datables du Moyen Empire, la première fut retrouvée entre les secteurs $\mathrm{N}_{2}$ et Ni3. Il s'agit d'un bord de jarre fabriqué en Marl $A_{3}$ qui, en raison de sa pâte et de sa morphologie, peut être daté du milieu de la XI $\mathrm{XI}^{\mathrm{e}}$ dynastie au milieu de la XII dynastie $^{26}$ (pl. 2. Medi5-I6I). Les deux autres tessons (pl. 2 Medi5-IOI et Medi5-I57) n'ont pas une datation aussi assurée. Il pourrait s'agit d'une figurine animale dont ne subsiste qu'une partie du cou, et de base d'un petit vase pointu fabriqué au tour lent et dont la base fut terminée à la main. La datation proposée repose sur leur pâte, $\operatorname{Marl} A_{3}$, qui est certes bien connue pour les époques postérieures, mais est surtout utilisée pendant le Moyen Empire.

Les fragments pouvant être datés de la Deuxième Période intermédiaire sont plus divers. Tout d'abord, il faut remarquer la présence de récipients décorés, comme un fragment d'assiette de grand diamètre, ainsi que le fragment de l'épaule d'une jarre, tous les deux avec une décoration incisée en forme de wavy lines et fabriqués en pâte $\operatorname{Marl} B$ (pl. 3. Medı4A-2 ${ }^{27}$ et pl. 4. Medi4A-26 ${ }^{28}$ ). Apparaissent également plusieurs types de jarres fabriquées en pâte Marl $A_{4}$ var. I et $\operatorname{Marl} B$ qui imitent des jarres de stockage typiques du Fayoum, normalement fabriquées en $M a r l C$ (pl. 4 Medi5-I62 ${ }^{29}$ et Medi5-I59). Plusieurs fragments de moules à pain sont également attestés à la Deuxième Période intermédiaire, mais sont en pâte locale mixte ou en calcaire (pl. 3. Medı4b-45; Medi4b-43 et Medi4a-65 ${ }^{30}$ ). La présence d'un bord de récipient de coction de type Kerma ou Pan Grave ${ }^{3 \mathrm{I}}$ avec une décoration incisée est également très représentative de cette époque (pl. 3 Medi4a-56).

24 Ce matériel a été pris en considération dans le cadre de la thèse de doctorat de Z. Barahona-Mendieta: BarahonaMendieta 2016.

25 Sur ces questions voir BarahonaMendieta 20i6; Relats Montserrat 2Oi6b et Relats Montserrat 2Oi6a.

26 Proche de Schiestl, Seiler 20I2, type II.B.2.d, fig. 6, p. 424 (fin de la XI ${ }^{\mathrm{e}}$, début de la XII ${ }^{\mathrm{e}}$ dynastie) ; type II.C.3.A, fig. 3-4, p. 445 et fig. 7-8, p. 446; Rzeuska 20II, fig. $5 \mathrm{D}$, famille I, type 3 , fig. $7 \mathrm{a} / \mathrm{b}$ (XII ${ }^{\mathrm{e}}$ dynastie).
27 Bourriau 1990, fig. 4.I.I5, en Nile $\mathrm{B}_{2}$; bols similaires fig. 4.2.6-7 en Nile $C$; Bourriau 20I0, fig. 6, $\mathrm{D}_{3}$, en Nile $C$; Bourriau 1997, fig. 6.I2, 4. Fin de la XII ${ }^{e}$ dynastie; proche de JACQUET-GORDON 2OI2, fig. I6b (Deuxième Période intermédiaire).

28 Aston 2007, fig. I9, $8947 \mathrm{~K}$; BOURriau I990, fig. 4.4, I5; BourriaU 20IO, fig. I4 D.; BUDKA 2006, fig. 6.2. 29 Bourriau I990, fig. 4.4.4, 6, en Marl B; Semblable à JacquetGordon 20I2, fig. 20n. Possible imitation de jarres de stockage en Marl C:
Bourriau, Op de Beek, Meyer 2005, fig. I8 a-b; Aston, BADER 2009, fig. 8.79. 30 Marchand 20I2, fig. 3.2I, C.I68063-I/2, Niveau IV, fig. 3.26, C. I68053-IO, Niveau V; JACQUETGORDON 2012, fig. 6b N, fig. I3g; JACQUET-Gordon 1981, fig. 4, type C; BUDKA 2006, fig. I.4.

31 La distinction entre ces deux catégories est assez complexe et, comme l'affirme S. Marchand, il s'agit d'un sujet propre aux spécialistes de ce type de céramique: Marchand 20I2, p. 78-79. 
Enfin, certaines pièces datent manifestement du tout début de la XVIII ${ }^{\mathrm{e}}$ dynastie, parmi lesquelles une anse d'amphore avec enduit rouge et décoration peinte en noir de forme ovale (pl. 6 Medi5-I ${ }^{32}$ ), un bol avec un enduit rouge poli (pl. 5 Medi5-SPII-8 ${ }^{33}$ ), un support annulaire (pl. 6 Medi4A-5I ${ }^{34}$ ) et un bol en forme de cratère (pl. 5 Medi5-I6 $5^{35}$ ).

\begin{tabular}{|c|c|}
\hline Moyen Empire & Medi5-I6I; Medi5-IOI; Medi5-I57 \\
\hline Deuxième Période intermédiaire & 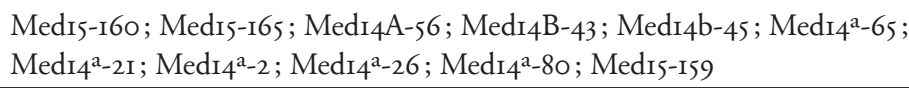 \\
\hline XVIII ${ }^{\mathrm{e}}$ dynastie & Medi5-I; Medi4 ${ }^{a}-51 ;$ Medis-SPII- 8 ; Medi5-I65 \\
\hline Époque Ramesside & Medi5-I89 \\
\hline
\end{tabular}

TABL. 3. Récapitulatif chronologique de la zone $\mathrm{P}_{9} / \mathrm{Mir}_{\text {et }} \mathrm{O}_{12} / \mathrm{M}_{2} 3$.

\section{Tribune}

\section{Secteur $\mathrm{P}_{2} / \mathrm{O}_{3}$ (tribune)}

Cette zone présente une densité très faible en céramique qui s'accroît néanmoins en s'éloignant des limites du secteur. La topographie générale prend la forme d'une cuvette entourée de butes sur trois de ses côtés qui peuvent être le produit des fouilles de $\mathrm{Cl}$. Robichon et A. Varille en 1936 dans ce secteur ${ }^{36}$. Les butes, aujourd'hui visibles, seraient les cavaliers de déblai sur les côtés sud, est et ouest du rectangle fouillé.

Les tessons couvrent une phase chronologique allant de la Basse Époque à l'époque byzantine. Cependant, la Basse Époque et l'époque ptolémaïque sont bien plus représentées du point de vue quantitatif. Nous pouvons citer le bord d'une assiette en $M a r l A_{4}$ var. 2 typique des $X X V^{e}-X X V I^{e}$ dynasties, tout à fait semblable à ceux qui ont déjà été documentés dans la production céramique des fours du sud du kôm (pl. II. 492IKd-4 ${ }^{37}$ ); une jatte décorée avec des traces de peinture violète de la fin de l'époque ptolémaïque et du début de l'époque romaine (pl. I2. Medi5-65 ${ }^{38}$ ); un bord de petite jarre ptolémaïque d'inspiration levantine (pl. I3. Medi52I ${ }^{39}$ ); un bord d'amphore romaine du type de celles qui ont également été documentées dans d'autres zones de Médamoud (pl. I4. Medi5-A8 ${ }^{40}$ ); et un bord d'assiette byzantine (IV-V $\mathrm{V}^{\mathrm{e}} \mathrm{s}$ ) fabriqué en pâte d'Assouan et décoré avec un engobe orange et des bandes blanches et noires semblables aux fragments découverts par F. Bisson de La Roque dans la zone du temple (pl. I5. $558 \mathrm{I}-\mathrm{II}-\mathrm{M} 4264^{4 \mathrm{I}}$.

32 Bourriau 2OIO, fig. I4g, $34 \mathrm{~m}$.

33 Bourriau 2OIO, fig. 3I, 4.I2.4.

34 Bourriau 2OIO, fig. 28, I5I.IO.

35 Similaire à Bourriau 20IO, fig. 25, 7.6.I.

36 Sur ces fouilles voir Relats MONTSERRAT 2OI6b.

37 Barahona-Mendieta 20I4, fig. 7. 38 Barahona-Mendieta 2017 , fig. 4.II. Par rapport aux pâtes calcaires de l'époque ptolémaïque et romaine à Médamoud: Barahona-Mendieta 2017 , p. 34.

39 SChreiber 2003, pl. 9, IIO-I23. La lèvre que nous présentons ici est surcuite et déformée ce qui nous permet de le considérer comme un déchet de coction - correspondant ainsi parfaitement à la production de céramique locale à l'époque ptolémaïque.
40 Amphore du type AE.3.5 (I-III d. C) : Dixneuf 20II, fig. o9-IIo. 41 Assiette découverte par F. Bisson de la Roque en 1929 entre la porte de Tibère et les kiosques. Sur ce type d'assiettes et sa chronologie voir RodzIEwICZ 2005, fig. I7. 


\begin{tabular}{|l|l|}
\hline Basse Époque & $492 \mathrm{I} \mathrm{Kd}-4$ \\
\hline Époque ptolémaïque & Med20I5-65; Medi5-2I \\
\hline Époque romaine & Medi5-A8 \\
\hline Époque byzantine & 558I-II M4264 \\
\hline
\end{tabular}

Tabl. 4. Distribution chronologique des céramiques du secteur $\mathrm{P}_{2} / \mathrm{O}_{3}$.

\section{Secteurs au sud du temple}

\section{Secteur $K_{4} /$ L9 (grande zone)}

Cette zone est la plus grande à avoir été prospectée au cours de notre campagne (fig. I7). Par rapport à la zone précédente, la densité de tessons de céramique est plus grande et plusieurs indices clairs suggèrent la présence d'ateliers de production de céramique. Ces derniers sont reconnaissables par la présence de briques vitrifiées provenant de parois de fours, ainsi que par une grande concentration de déchets de coction. À titre d'exemple, nous pouvons évoquer la paroi de four constituée de deux briques vitrifiées sous l'effet de la chaleur et contre lesquelles sont encore collés plusieurs fragments de céramique, tous cassés, surcuits et à pâte calcaire. Ces tessons sont cependant trop déformés pour pouvoir dater l'époque d'utilisation de ce four. La paroi de four fut trouvée sur le sol à cheval entre la zone L5 et K6 (fig. 16).

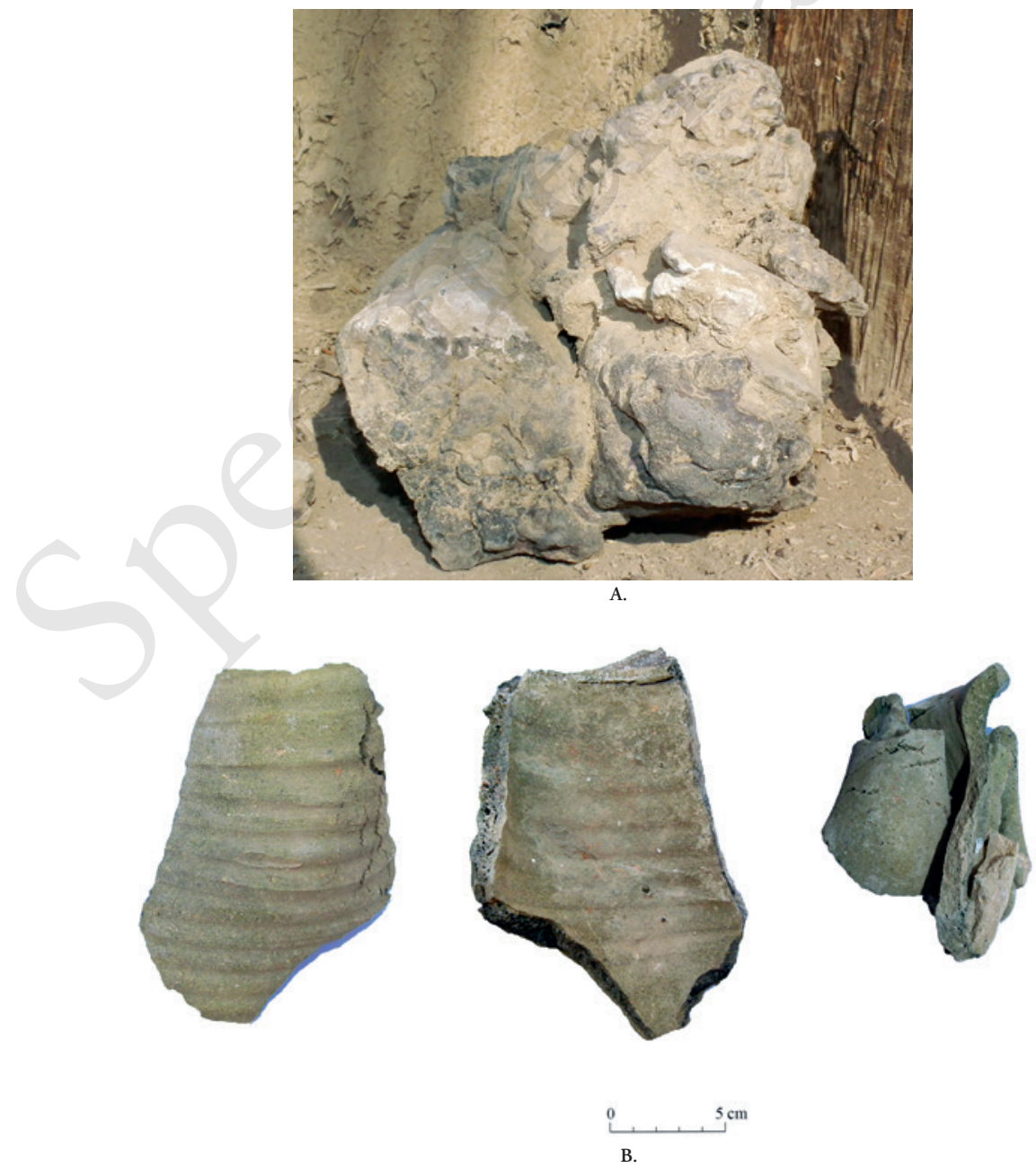

Fig. I6. A. Paroi de four, trouvée sur le sol de L5/K6. (c) Z. Barahona-Mendieta. B. Exemples de ratées de cuisson trouvées sur le kôm. 
Les céramiques sont très hétérogènes du point de vue chronologique. Tout d'abord, de la céramique du début de la XVIII e dynastie a été reconnue, mais elle est plus abondante au nord de L7/L8 et tout particulièrement autour d'un petit monticule. Il est possible qu'il soit formé par le cavalier de déblais de sondages effectués par $\mathrm{Cl}$. Robichon et A. Varille autour de l'angle sud-ouest du temple. En effet, ce type de céramique est identique à celui qui fut retrouvé dans les fours au sud-ouest du temple actuel ${ }^{42}$.

D'un autre côté, les bords de Meat Jars sont très abondants dans tout ce grand secteur. Plusieurs exemples sont surcuits, ce qui nous incline à penser que ce type de récipient fut fabriqué dans ce secteur. Sa production s'étend de la deuxième moitié de la XVIII ${ }^{\mathrm{e}}$ dynastie à la fin de la Troisième Période intermédiaire, devenant très abondante à l'époque ramesside où elle subit diverses modifications. Cela est visible avec les fragments pl. 7 Medi5-75; Medı5-35; Medis-II ; Medi4-2 et Medis-K9-2 ${ }^{43}$.

Il faut également signaler une accumulation de fragments d'amphore, dont plusieurs surcuits et déformés dans les zones $\mathrm{L}_{4}$ et $\mathrm{L}_{5}$ qui imitent l'aspect des amphores du sud-est de la mer Egée ${ }^{44}$ comme celles de Cnide, Rhodes ${ }^{45}$, Chios ou même de Chypre ${ }^{46}$. D’après la documentation archéologique des sites de référence, ces amphores apparaissent pendant la première moitié du $\mathrm{III}^{\mathrm{e}}$ s. av. J.-C. pour disparaître au $\mathrm{II}^{\mathrm{e}}$ s. av. J.C 47 . Il faut également remarquer la présence de nombreux bords d'amphore qui imitent d'autres modèles levantins comme les «Amphores torpedo ${ }^{8}$ » (pl. I3. Medi5-I86; Medi5-K9-24; Medi5-Io6 - ces trois fragments sont surcuits et vitrifiés) qui ont été à l'origine de certains modèles d'amphores d'époque perse et ptolémaïque ${ }^{49}$. La présence systématique de fragments surcuits et déformés/vitrifiés nous montre de façon certaine qu'une production d'imitations grecques et levantines a eu lieu dans ces secteurs.

Pour terminer il faut signaler que ce secteur a également livré de très nombreux fragments de Basse Époque qui peuvent probablement provenir des fours trouvés pendant un sondage au sud du kôm en 1928 (cf. infra). La présence de ce type de céramique sur tout le site témoigne de l'abondance de cette production (pl. IO. Medi5-I96; Medi5-I72; pl. II. Medi5-202 ; Medi5-I2I ; Medis-K9-Io).

\footnotetext{
42 Barahona-Mendieta 2OI4, fig. I-6.

43 Bourriau 20io, p. I4I. Aston 2008, fig. 25 , groupe 25 ; fig. 26 , groupe 33 .

44 Barahona-Mendieta 20I7, fig. 20, pl. I3. Medi5-7.

45 Parallèlement à cette production locale, à Médamoud est également
}

attestée la présence d'importations rhodiennes grâce à la présence d'un sceau d'amphore rhodienne datée de I27 av. J.C.: Barahona-Mendieta 20I5. 46 Pl. i3. Medis-ib. Amphore à anses de panier. Defernez, Marchand 2006, fig. 2.

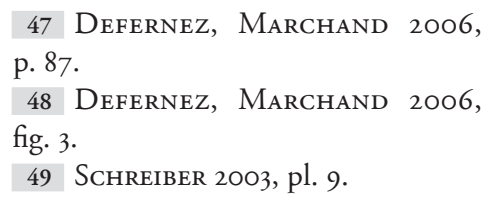

47 Defernez, Marchand 2006, p. 87 . 48 Defernez, Marchand 2006, fig. 3 . 49 SChreIber 2003, pl. 9. 


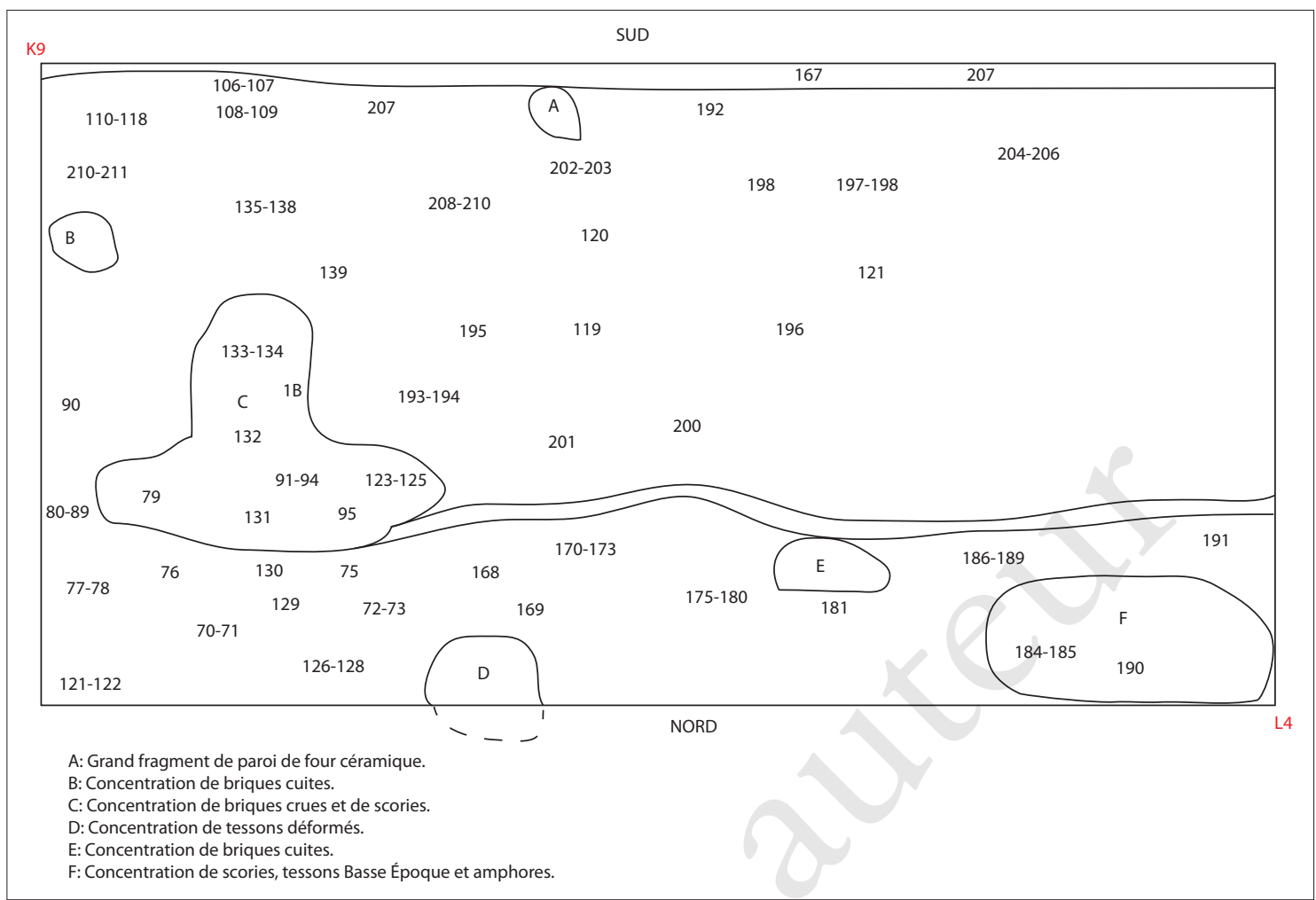

Fig. 17. Croquis du secteur $\mathrm{L}_{4} / \mathrm{K}_{9}$.

\begin{tabular}{|c|c|}
\hline XVIII ${ }^{e}$ dynastie & $\begin{array}{l}\text { Medi5-I20; Medi5-I95; Medi5-I73; Medi5-2Io; Medi5-K9-I; Medi5-96; } \\
\text { Medi5-I35; Medi5-K9-25; Medi5-79; Medi5-IO8; Medi5-93; Medi5-74 }\end{array}$ \\
\hline Époque ramesside & Medi5-I89; Medi5-20I \\
\hline Basse Époque & Medi5-K9-2; MedI5-I96; Medi5-202; Medi5-I2I; MedI5-I72; Medi5-K9-IO \\
\hline Époque ptolémaïque & $\begin{array}{l}\text { Medi5-K9-33; Medi5-L5-23; Medi5-K9-24; Medi5-I; Medi5-I86; Medi5-II3; } \\
\text { Medi5-I06; Medi5-209 }\end{array}$ \\
\hline
\end{tabular}

TABL. 5. Distribution chronologique des céramiques du secteur $\mathrm{K}_{4}-\mathrm{K}_{9} / \mathrm{K}_{9}-\mathrm{L} 9$.

\section{Secteur LIo/GI2}

Ce secteur couvre également une grande surface - dépourvue de végétation - mais qui, par la proximité avec les habitations modernes, est très régulièrement recouverte par des déchets.

Les céramiques sont très hétérogènes du point de vue chronologique. Les fragments les plus anciens (pl. 3. Medi5-35 50 et Medi5-5 ${ }^{5 \mathrm{I}}$ ) remontent à la fin de la Deuxième Période

50 Bourriau I990, fig. 4.3, I7; KopetZKY 20I2, fig. 6, 57; Aston 2004, pl. 80, groupe 72, 232.

51 Bourriau I990, fig. 4.I.I4, en Nile $C$ et sans décoration mais avec un profil très proche; fig. 4.3.22, en Marl $B$ avec un profil proche mais sans décoration; fig. 4.5.3, bol avec des digitations et l'application en Marl B. BudKa 2006, fig. 18.4-5, grandes jattes en Nile B2 avec une décoration détaillée de visages hathoriques sur leurs bords. Bourriau 20IO, fig. I2, 4.I8.3, Nile B2, fig. 25, 4.I8.3, Nile B2, fig. 30, 4.18.4, Marl B, fig. 39, 4.I8.3, Nile B2, fig. 64, 4.I8.3, Nile B2, fig. 8I,
4.I8.3, Nile B2. JacQuet-Gordon 2OI2, fig. I9 d-e. Tyson-Smith 20I2, fig. Iod. Aston 2004, groupe 22I, pl. 214-215. Marchand 20I2, fig. 3.24, C.I68I3-5. Seiler i999, Abb. 48. Marchand, SOUKIASSIAN 20I0, groupe 60, phase 4. Brunton 1930, pl. XII, 9g. SeILer I997, fig. 2. 
intermédiaire et furent retrouvés au sud-ouest de la zone, approximativement en Gio et GiI. Ils peuvent être mis en relation avec d'autres fragments présentant la même chronologie du secteur G9 (pl. 3. Medi5-I52 et Medi5-I4752). Cependant ces mêmes zones ont également livré de nombreux fragments de Basse Époque qui pourraient provenir en réalité des grands dépôts de céramique retrouvés au sud du kôm, là où se trouvaient les fours de cette époque. Il est probable qu'il faille également considérer comme résiduels certains fragments datés du Nouvel Empire et de l'époque ramesside qui furent trouvés dans la moitié nord-ouest de la zone, étant donné qu'ils correspondent à la céramique produite à cette époque dans des zones plus proches du temple.

Pourtant, la plus forte concentration de tessons est datée de l'époque romaine, notamment des godets de noria (pl. I4. Medi5-I9b, Medi5-20b et Medi5-2753) et des petites marmites (pl. I4. Medi5-354) bien attestées de l'époque impériale à l'époque tardo-romaine. Il serait envisageable de relier ces restes de production avec des briques cuites, scories et des cendres observables dans les secteurs Iı2 et Ji2.

Il faut également mettre en exergue que le grand secteur dégagé et de forme rectangulaire qui s'étend au sud de KI2, présente majoritairement des fragments d'époque romaine et, de manière résiduelle, d'époque byzantine.

\begin{tabular}{|l|l|}
\hline Deuxième Période intermédiaire & Medi5-35 \\
\hline XVIII ${ }^{e}$ dynastie & Medi5-I5; Medi5-4; Medi5-I8 \\
\hline Époque ramesside & Medi5-3I; Medi5-32; Medi5-2 \\
\hline Basse Époque & Medi5-I5-9; Medi5-IO2; MedI5-I0o; Medi5-8; Medi4A-IOI; Medi5-6 \\
\hline Époque ptolémaïque & Medi5-2I; Medi5-I4; Med15-7 \\
\hline Époque romaine et byzantine & Medi5-20b; Medi5-I9b; Medi5-27; Medi5-3 \\
\hline
\end{tabular}

TABL. 6. Distribution chronologique des céramiques du secteur Lio/Gı2.

\section{Secteur H6/G9}

Ce secteur peut être clairement mis en rapport avec une grande production de céramique qui fut déjà découverte en 1928, mais qui n’a quasiment pas été publiée par les anciens fouilleurs 55 . Il se caractérise par une grande densité de tessons ainsi que par le nombre de scories et de briques cuites (fig. I8). Là encore, certaines céramiques de la Deuxième Période intermédiaire

52 Schiestl, Seiler 2OI2, groupe I.G.5, p. 327; I.G.7.c, p. 336; JACQUET-GORdON 2OI2, fig. 3Ih-K; Marchand, Soukiassian 2OIO, groupe I9A, phase I-2; groupe 2I, phase 2.

53 Pierrat-Bonnefois i996, fig. I4; LaUfFraY I995, fig. 53, I34; DaVID 20I3, fig. 4.I7.
54 Lauffray 1995, fig. 45, 2I4; Tomber 2006, fig. I.3I, 50/406. Il est probable que ces céramiques appartiennent à la même production que les jattes Medis-Ui3-I2, Medis-A3 et que d'autres céramiques bien connues en Haute Égypte de ce même type de pâte calcaire comme PierratBonnefors 1996, fig. 15, 56-58, étant donné que de nombreux fragments de ce type ont été retrouvés.

55 Bisson de La Roque i93I, p. 4; Bisson de La Roque i933, p. 34; Barahona-Mendieta, Relats-Montserrat 2017 ; Barahona-Mendieta 20I4; Barahona-MENDIETA 2017. 
(pl. 3. Medi5-I47 ${ }^{56}$ et Medi5-I52) sont reconnaissables dans le secteur G9 et peuvent être mises en rapport avec celles retrouvées au sud du secteur Gio. Dans sa partie sud également ont été localisés plusieurs bords de jarres de la Troisième Période intermédiaire, dont l'un d'entre eux était complètement déformé sous l'effet de la cuisson (pl. 9. Medi5-I48 57 et Medi5-I49 ${ }^{58}$ ).

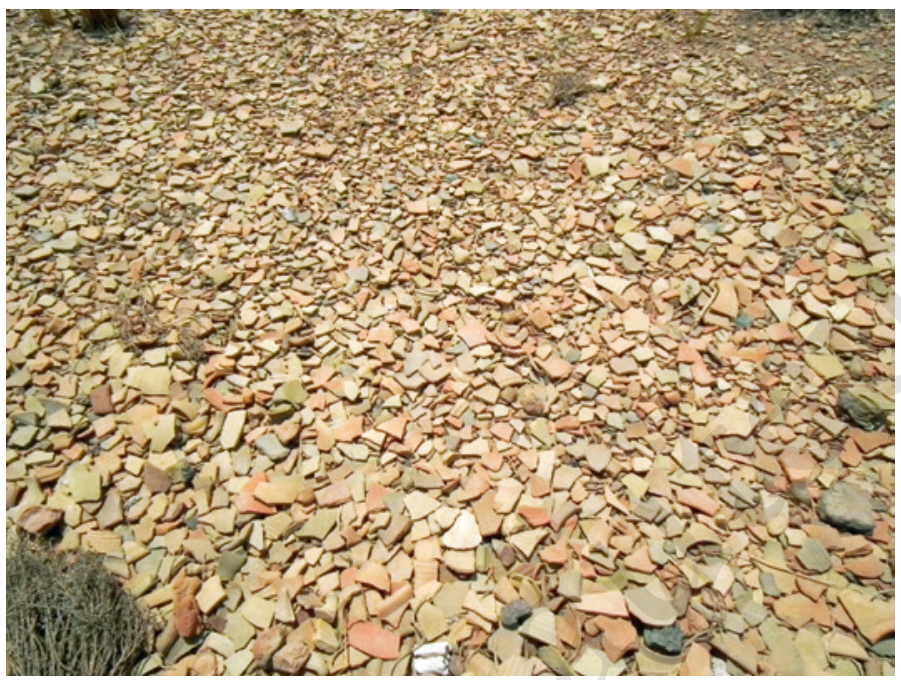

Fig. I8. Céramique de surface, avec tessons surcuits et briques vitrifiées.

La céramique la plus répandue dans ce secteur date de la Basse Époque, avec une surreprésentation d'époque perse (pl. Io. Medi5-8 59 et pl. II. Medi5-IO2 ${ }^{60}$ ). C'est visiblement dans cette zone que se concentra la production de céramique de cette époque, même si la production dut se poursuivre jusqu'à la fin de l'époque ptolémaïque comme le prouvent les tessons surcuits retrouvés dans ce secteur (pl. I2. Medi5-IO; Medi4 B-3 ${ }^{61}$ ).

De façon résiduelle, nous avons trouvé un bord d'African Red Slip Ware importé de Tunisie de la forme Hayes 9I (pl. I5. Medi5-4I) daté de la seconde moitié du IV ${ }^{\mathrm{e}}$ s. et de la première moitié du ve s. ${ }^{62}$.

\footnotetext{
56 Schiestl, Seiler 20I2, groupe I.G.5, p. 327, I.G.7.c, p. 336; JACQUET-GORDON 2OI2, fig. 3Ih-K, IIPI; Marchand, Soukiassian 20IO, groupe I9A, phase I-2, groupe 2I, phase 2 .

57 JACQUET-GORDON 2OI2, fig. 9I.d 58 JACQUET-GORDON 2OI2, fig. 9I.C; Aston 1999, pl. 21, n 617, 619, pl. 28, $\mathrm{n}^{\circ} 839$.

59 Masson 2007, pl. XVII, fig. 2.
}

60 JACQUET-GORDON 2OI2, fig. IO7.s; MAsson 20II, fig. 29; Aston 1999, pl. 65, $\mathrm{n}^{\circ}$ I9IO, pl. 7I, $\mathrm{n}^{\circ} 2029$.

61 Barahona-Mendieta 20I7, fig. I6. 62 Bonifay 2004, fig. 95, sigille type 48, 49 . 


\begin{tabular}{|c|c|}
\hline Deuxième Période intermédiaire & Medi5-I47; Medis-I52 \\
\hline XVIII dynastie & Medi5-I; Medi5-20 \\
\hline Époque ramesside & Medi5-I5I; Medi5-35 \\
\hline Troisième Période intermédiaire & Medi5-I53; Medi5-I48; Medi5-I49; Medi5-6a \\
\hline Basse Époque & Medi5-2I; Medi5-8; Medi5-22a ; MedI5-I39; Medi5-34; Medı5-46 \\
\hline Époque ptolémaïque & Medi5-Io; Medı4b-3; Medi5-44; Med20I5-6; Medi5-G7-33 \\
\hline Époque romaine & Medi5-3I; Medi5-30 \\
\hline Époque Byzantine & Medi5-4I \\
\hline
\end{tabular}

TABL. 7. Distribution chronologique des céramiques du secteur G7/G9.

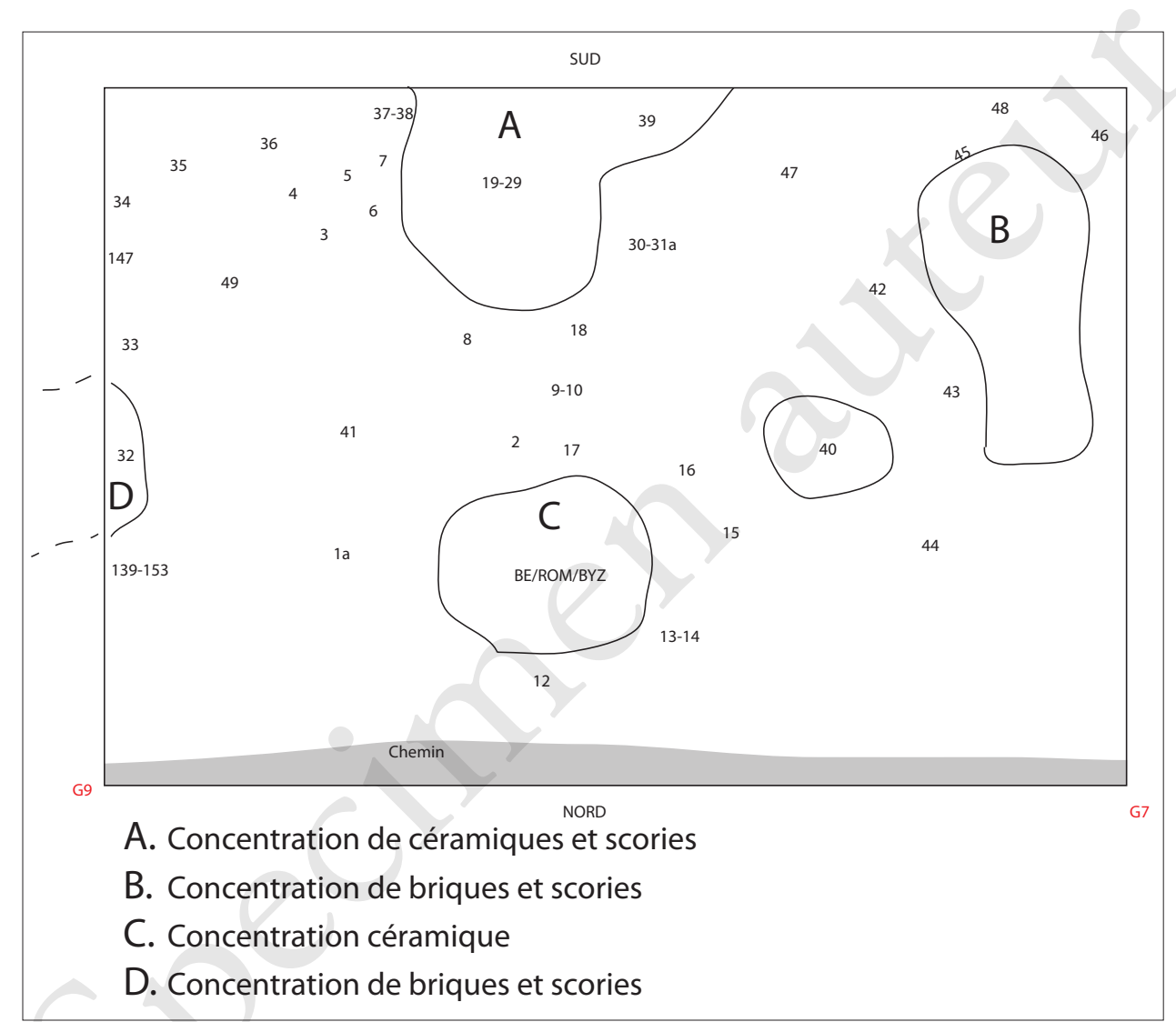

Fig. 19. Croquis de localisation de la céramique de la zone G7/G9. 


\section{SYNTHÈSE ARCHÉOLOGIQUE}

À ce stade de notre réflexion, deux éléments peuvent être mis en exergue: d'une part, le site présente une richesse exceptionnelle en termes de matériel céramique faisant de Médamoud un centre de production important, avec de nombreux déchets de production. D'autre part, les prospections géophysiques prouvent l'existence d'une accumulation de vestiges difficilement lisibles. Pour aboutir à une lecture archéologique, nous devons combiner les résultats des fouilles anciennes et ceux de la prospection céramologique, avec le type de réponse géophysique que pourraient avoir a priori les vestiges (tabl. 2). Cette réflexion devra être menée à l'échelle du site, en prenant en compte l'emplacement des zones par rapport au temple (fig. 20).

Une remarque liminaire s'impose cependant. Les cartes d'interprétation géophysique ont signalé plusieurs réponses suffisamment caractéristiques pour être mentionnées, comme les éléments métalliques non magnétiques, mais qui ne sont pas interprétables par manque d'information $^{63}$. Pour éviter de multiplier les hypothèses, nous focalisons donc notre discours sur les trois axes de réflexion énoncés en introduction.
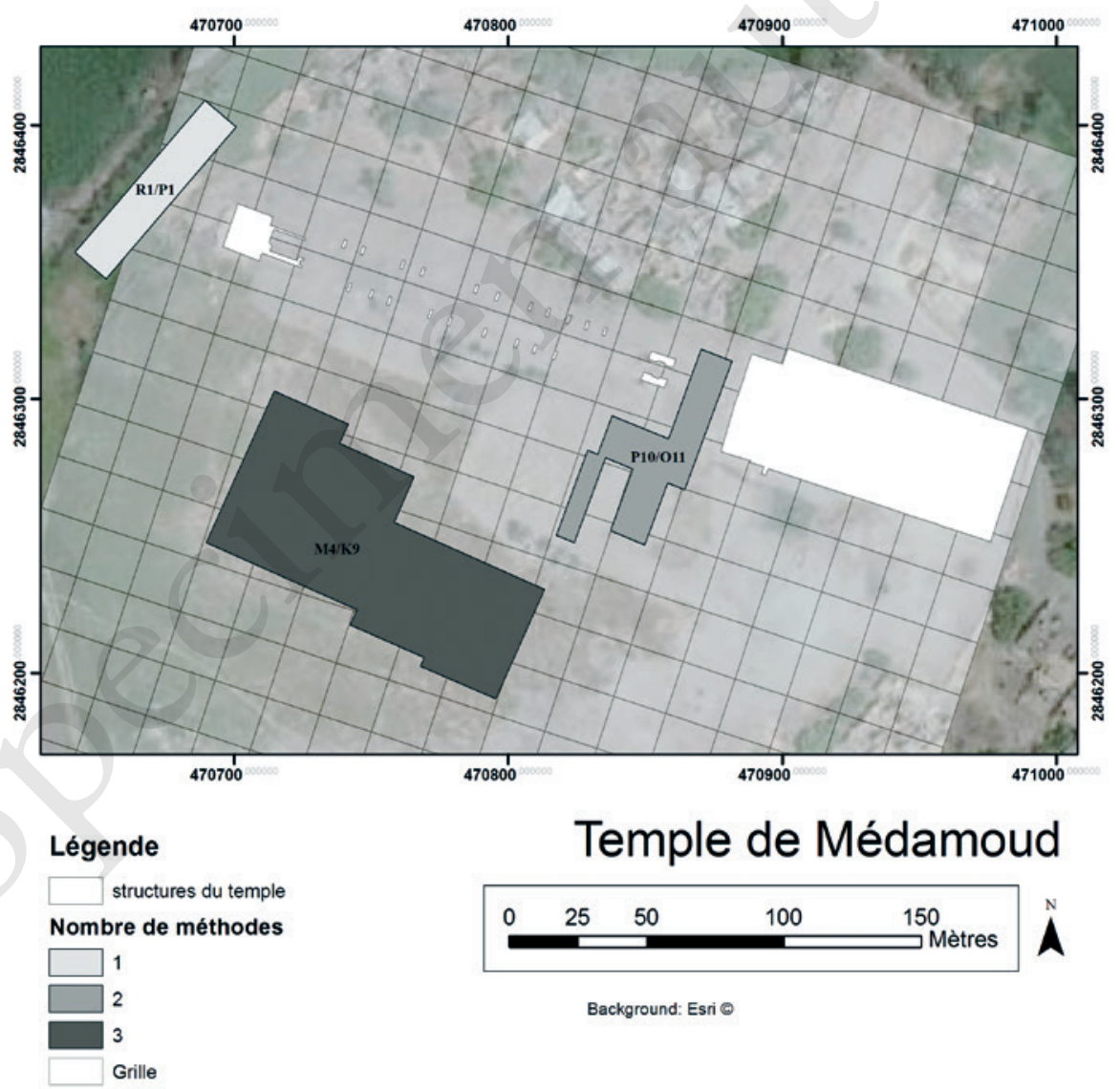

Temple de Médamoud

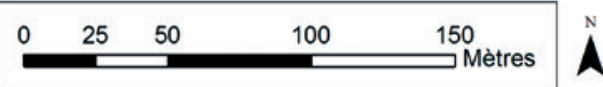

Background: Esri $\odot$

FIG. 20. Emplacement des zones de prospection discutées dans la synthèse.

63 Il pourrait s'agir, par exemple, aussi bien de déchets métalliques enfouis en profondeur qu'un outil en bronze. La seule certitude est qu'ils ne sont pas en fer mais dans un autre alliage (bronze, cuivre). 


\section{La liaison entre Médamoud et le Nil: zone RI/PI (canal)}

À l'extrémité ouest du dromos fut érigée une tribune, reliée à ce dernier par une rampe d'accès ${ }^{64}$. Il s'agit d'une construction appareillée en grès de $16,20 \mathrm{~m} \times 13,40 \mathrm{~m}$, dont le niveau supérieur se trouve à $+\mathrm{I}, 70 \mathrm{~m}$ au-dessus de l'allée ${ }^{65}$. Nous postulons, comme le fit F. Bisson de La Roque, que la datation de cette tribune peut être rattachée à celle du dromos. Ce dernier fut d'abord daté d'époque augustéenne, en rapport avec la construction de la porte de Tibère ${ }^{66}$, avant d'être attribué à Ptolémée III en raison d'une inscription retrouvée dans un remploi byzantin à proximité de la rampe ${ }^{67}$. Aucun de ces arguments n'est cependant concluant. Le style des sphinx, seules constructions in situ peut correspondre à une fourchette couvrant les règnes de Nectanébo Ir à celui des premiers Ptolémées ${ }^{68}$. Dans tous les cas, l'existence d'un tel aménagement, similaire à d'autres temples thébains, implique un accès à l'eau au début de l'époque ptolémaïque et sûrement augustéenne.

Cet aménagement cependant ne va pas de soi puisque le temple de Médamoud se trouve à $4,9 \mathrm{~km}$ à l'est du cours actuel du Nil. Pour résoudre cette contradiction, les fouilleurs ont postulé l'existence d'un canal creusé à main d'homme qui aurait uni la tribune de Médamoud au Nil en passant par Karnak-nord, sans trouver des traces de son existence sur le terrain ${ }^{6}$. F. Relats a déja montré que cette hypothèse se fonde, d'une part sur le lien religieux entre Karnak-nord et Médamoud, et d'autre part sur le tracé du réseau topographique contemporain des fouilleurs ${ }^{70}$. Il n'est donc aucunement assuré que Médamoud et Karnak-nord aient été reliés. Malgré tout, il devait bien y avoir un canal reliant Médamoud au Nil, dont le tracé n'est pas connu, faute d'indices archéologiques. Par ailleurs, F. Bisson de La Roque a développé une réflexion assez confuse sur les niveaux du Nil pour comprendre si la tribune pouvait effectivement être en contact avec l'eau du fleuve. Il conclut qu'il devait y avoir un bassin devant la tribune qui « aux autres saisons que celle de l'inondation, ne devait pas toucher la façade ouest de la tribune, où rien ne laisse supposer un accostage ${ }^{71}$. En effet, à la différence de Karnak, aucun passage de corde d'amarrage n'a été découvert; il faut cependant signaler que ces éléments se trouvent

\begin{abstract}
64 Pour une description architecturale: Médamoud I93I/I932, p. I-3, 9-13. 65 F. Bisson de La Roque, reprenant une pratique encore courante en égyptologie, a entretenu une confusion sur le rôle de cette construction, l'appelant tantôt tribune, tantôt quai, voire même quai-tribune, avant de se corriger et de distinguer la tribune des aménagements qui permettaient l'accès à l'eau: "Abusivement on a désigné du nom de quai, la tribune située à l'extrémité des dromos, et sur laquelle le clergé devait se tenir pour voir arriver ou partir les processions par eau ", Médamoud I93I/1932, p. 9.

66 Bisson de La Roque 1933, p. 9.

67 Bisson de La Roque I946, p. 43.
\end{abstract}

68 L'attribution des sphinx à Nectanébo I ${ }^{\text {er }}$ a été proposée par Cabrol 200I, p. I83, n. 29.

69 "Une avenue de sphinx conduisait de l'enceinte sacrée à un quai, où devait aboutir un canal unissant Karnak à Médamoud, pour les processions par voie d'eau ", Bisson de La Roque I933, p. I. Cette hypothèse avait été formulée quelques années auparavant lors des premières fouilles à Karnak-Nord par M. Pillet en 1924-I925 (Pillet I924; Pillet 1925). Ce dernier, à l'occasion du dégagement de la tribune et du dromos de ce temple (orientés vers le nord) affirmait que la tribune devait donner accès à un quai baigné par un canal plutôt qu'au fleuve lui-même. Ce canal devait trouver son origine entre Louqsor et Karnak, longer le Nil sur la rive droite, baigner le quai occidental de Karnak, puis le quai de Karnak-Nord et enfin se diriger vers Médamoud. Pour les structures découvertes à Karnak-nord: PM II, p. I-2; Pillet 1924, p. 5I-88; Pillet i925, p. I-24; VARILle I943, p. I; Gabolde-Rondot 1993, p. 248, n. 27; Cabrol 200I, p. 57I-579. Pour une présentation des activités archéologiques de Karnak-nord, voir l'exposé synthétique livré par CABrol 200I, p. 9-I2.

70 Pour développement complet, se reporter désormais pour Médamoud à Relats Montserrat 20i6a.

71 Bisson de La Roque i933, p. I3. 
habituellement sur les deux quais latéraux et pas sur la tribune elle-même ${ }^{72}$. Rien n'empêche donc que de tels aménagements aient été prévus à proximité de la tribune, mais qu'ils n'aient simplement pas été retrouvés à Médamoud. D’autre part, la réflexion de F. Bisson de La Roque sur les niveaux de l'eau peut être désormais corrigée: en se fondant sur les calculs effectués par $\mathrm{Cl}$. Traunecker et par J. Seidlmayer ${ }^{73}$, le niveau estimé de l'eau à l'époque ptolémaïque et romaine devait être suffisamment élevé pour atteindre la tribune ${ }^{74}$. De ce fait, la question du lien entre Médamoud et le Nil reste donc, encore aujourd'hui, ouverte.

Pour apporter de nouveaux éléments au débat, nous avons décidé de prospecter les abords de la tribune. La partie nord des cartes de conductivité électrique de la zone (fig. 3, 2I) montrent que se dessine une séparation nette entre des zones à faibles valeurs (en bleu) et à valeurs moyennes (en vert) qui pourrait correspondre à une limite de canal. La partie centrale (en rouge), beaucoup plus conductrice, est sûrement un effet des pratiques agricoles récentes et correspond assez bien à des limites parcellaires visibles en surface dont la marque peut se voir sur le signal par la bande moins conductrice autour du point (E470660, N2846370). La limite sud n'apparaît pas clairement et il est possible qu'elle se situe au-delà de la zone prospectée. Si nous nous fions à ces résultats, le canal ne se trouverait pas dans le prolongement direct de la tribune, mais arriverait au nord-ouest de celle-ci (fig. 2I). La nature du terrain devant cette dernière nous a empêchés de prospecter la zone qui lui est adjacente et nous ne pouvons ainsi savoir si le canal s'élargissait pour former un bassin. Même si nous n'avons découvert qu'une faible partie de son tracé, l'orientation globale nous invite à postuler une orientation ouest/est pour le canal qui joindrait ainsi directement Médamoud au Nil sans passer par Karnak-nord. Bien évidemment, il faudrait étendre les recherches au-delà de notre concession pour pouvoir confirmer cette hypothèse.

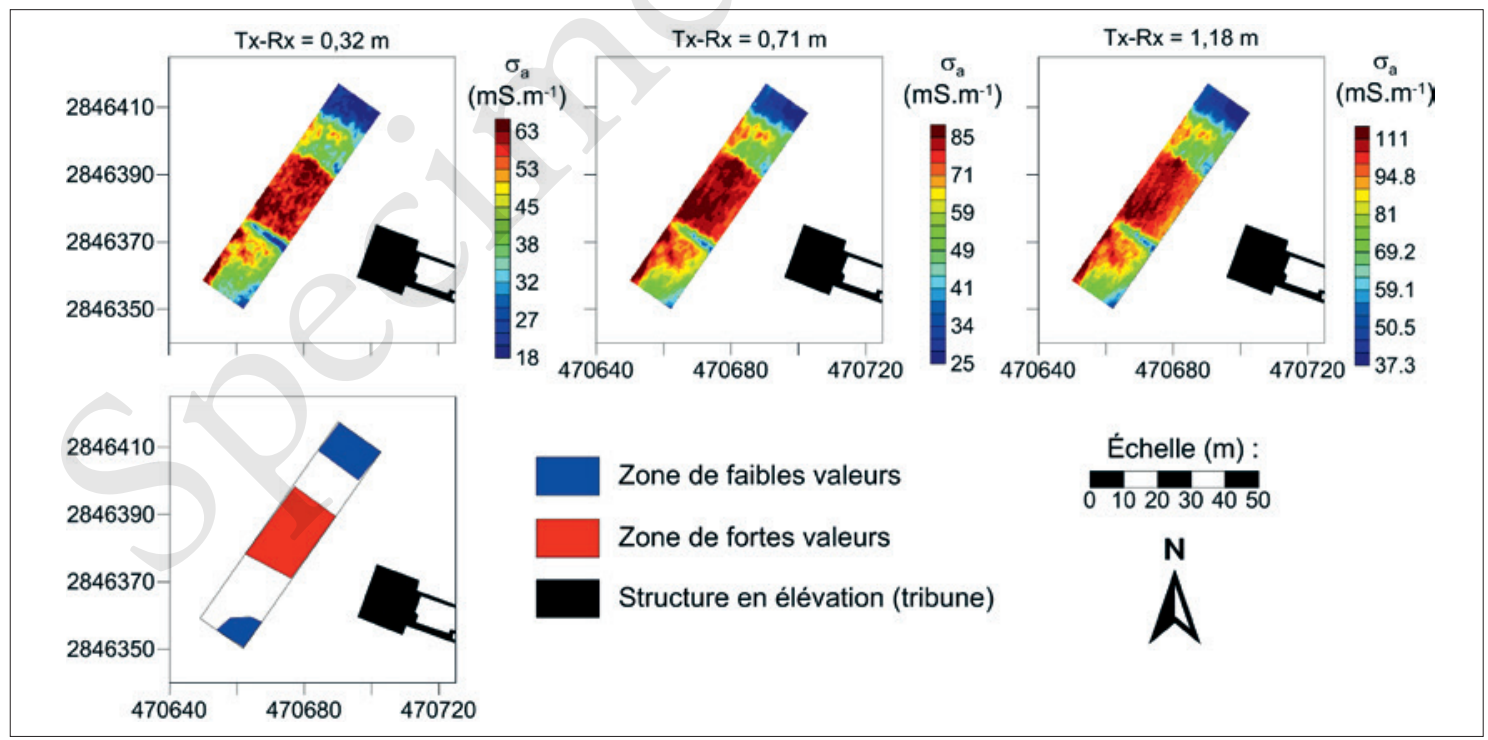

Fig. 2I. Synthèse des résultats de la tribune.

72 Lauffray i97I, p. IOI ; TrauneCKer I972, p. 195-236. 73 Traunecker i97I, p. I77-I96 (repris et corrigé par GabOLDE 1998, p. I96, n. 72) et Seidlmayer 200I, p. $63-73$.

74 Pour la démonstration complète:

Relats Montserrat 20i6a. 


\section{Le parvis du temple, le secteur sud-ouest et les murs de clôture: zone Pio/OII (poterne)}

Les abords du temple ptolémaïque sont marqués par un grand nombre de constructions témoignant des différentes phases du site ${ }^{75}$. À la différence des autres zones, nous possédons les plans issus des fouilles de F. Bisson de La Roque pendant lesquelles furent découverts deux murs de clôture (le mur augustéen dans lequel s'ouvrent la porte de Tibère ${ }^{76}$ et un mur antérieur appelé «mur de $9 \mathrm{~m}^{77}$ ), des fours à céramiques ${ }^{78}$ ainsi qu'une construction en brique cuite appelée la «maison carrée 79 ». Ces différents vestiges devraient $a$ priori répondre aux mesures géophysiques s'ils sont encore présents. Ainsi, une structure en terre ayant subi une cuisson (brique cuite) verra généralement sa susceptibilité magnétique augmenter (transformation d'oxyde de fer), pourra acquérir une aimantation thermorémanente et conduira moins bien le courant (perte d'eau et modification de sa structure). Les fours présenteront les mêmes caractéristiques, mais auront des réponses plus compactes spatialement que des structures bâties qui seront allongées, voire étendues. Par contre, la brique crue aura a priori une réponse différente (conductivité électrique plus importante, susceptibilité magnétique plus faible, pas d'aimantation rémanente). C'est pourquoi nous avons voulu superposer les plans anciens à nos propres résultats. Cependant, F. Bisson de La Roque publia trois plans différents de ce secteur, correspondant aux différentes campagnes de fouille (I929, I930 et I93I). Nous les avons combinés dans notre pl. I, en nous fondant sur les vestiges du temple qui sont présents sur tous les plans. Or, cet assemblage ne donne pas de résultats concluants puisque les deux murs de clôture ont un tracé différent à chaque fois (pl. I). Cela signifie qu'il y a une erreur dans au moins un des plans que nous ne pouvons pas corriger ${ }^{80}$. Ils peuvent donc être utilisés comme preuve de l'existence d'un vestige, mais pas pour le localiser précisément. C'est pourquoi, nous ne présenterons pas de superposition des plans avec les résultats géophysiques.

Nous pouvons néanmoins partir des vestiges découverts pour en établir les caractéristiques architecturales qui doivent avoir une réponse géophysique d'après les critères que nous avons précédemment exposés. Nous allons ensuite vérifier dans nos propres résultats si les secteurs où devaient se situer approximativement les vestiges présentent les signatures attendues. Nous regroupons les interprétations des propriétés mesurées dans la fig. 22.

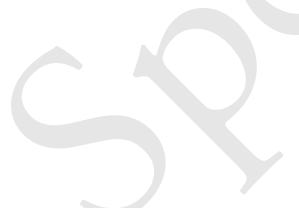

75 Nous appelons parvis du temple toute la zone comprise entre les kiosques et la porte de Tibère. Le secteur sudouest désigne l'angle sud-ouest inclus dans le mur de clôture augustéen.

76 Bisson de la Roque, Clère, Drioton I928, p. 5; Bisson De La RoQue, Clère 1929, p. 8.

77 Bisson de La RoQue i930, p. 9-I5; Bisson de La Roque i93i, p. 39-40.

78 Bisson de La Roque i93I, p. I9-2I.
79 Bisson de La Roque i93i, p. 8-9. 80 Nous n'avons aucun critère pour savoir quel plan est juste. Il se pourrait que l'erreur vienne du tracé du mur de $9 \mathrm{~m}$ dans le plan de 1929 (Bisson de La Roque 1930, pl. I). Il faudrait alors le faire pivoter par rapport aux kiosques pour qu'il corresponde au tracé du plan de i93o (Bisson de La Roque I93I, pl. I). Il est cependant également possible que ce soit le positionnement des kiosques et du lac qui soient faux dans le plan de 1930. Dans ce cas, c'est ce dernier plan qu'il faudrait faire pivoter. Il pourrait enfin s'agir d'un problème de mise à l'échelle entre le relevé archéologique des vestiges découverts et l'élévation du temple. Or pour géoréférencer les plans, nous devons nous appuyer sur des constructions dont l'emplacement est assuré, ce qui n'est pas le cas ici. 


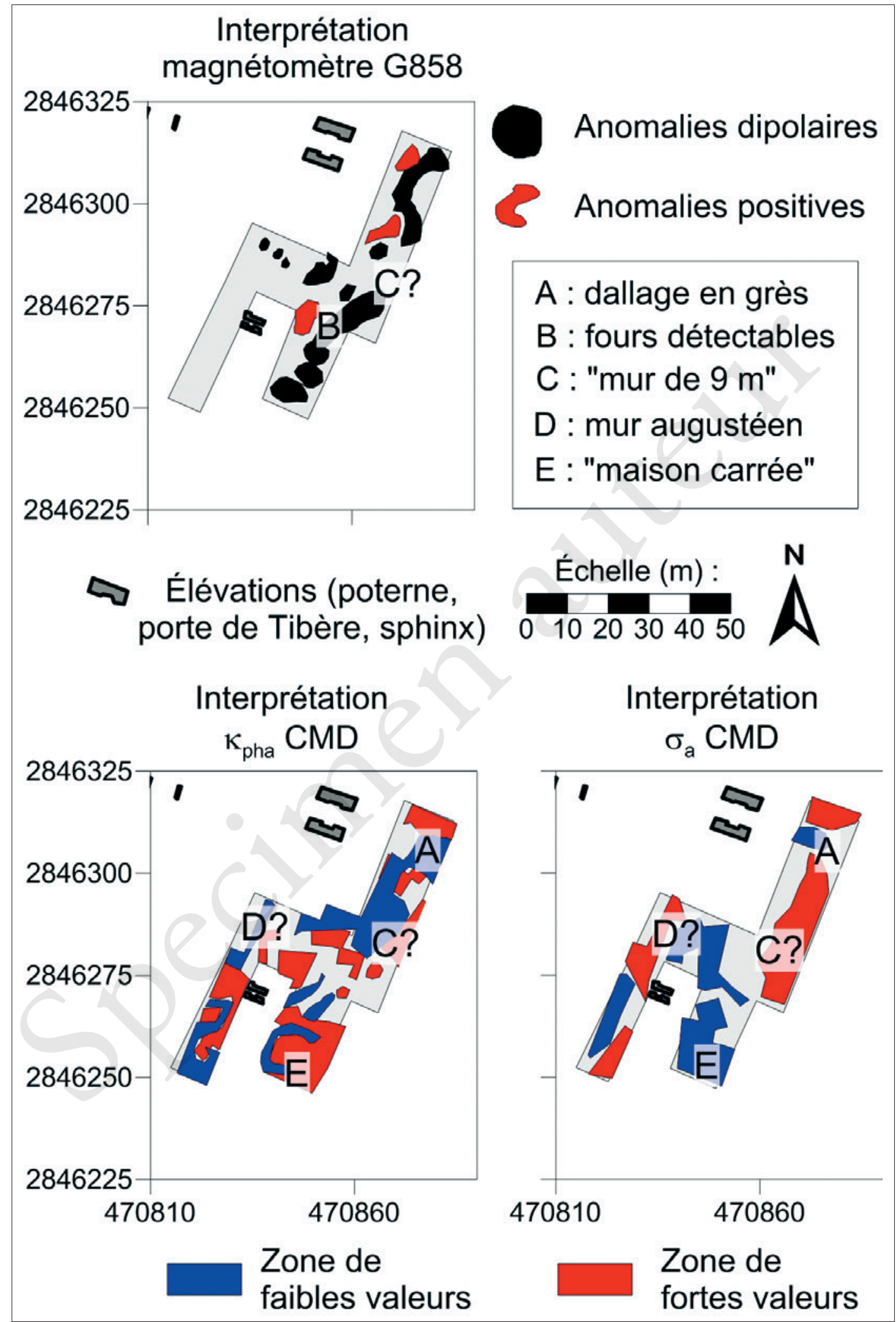

Fig. 22. Synthèse des propriétés géophysiques de la zone Pio/OıI. 


\section{Le dallage entre la porte de Tibère et les kiosques}

Le dallage se compose de blocs de grès. Parce qu'il est encore partiellement en place (pl. I), il est facilement reconnaissable dans les résultats géophysiques. De par son matériau, il se caractérise par de faibles valeurs en susceptibilité magnétique et en conductivité électrique. Au nord de la fig. 22, devant la porte de Tibère nous avons signalé par la lettre A le secteur correspondant à un tel résultat.

\section{Les fours}

À l'angle sud-ouest du mur de clôture augustéen, ont été retrouvés au moins 12 fours à céramiques (pl. I). L'étude céramologique a permis de les dater d'une fourchette chronologique couvrant la Deuxième Période intermédiaire et la seconde moitié de la XVIII ${ }^{\mathrm{e}}$ dynastie $^{8 \mathrm{r}}$. En raison du plan réalisé par F. Bisson de La Roque et la superposition de fours qu'il présente, deux phases se sont sûrement succédé (pl. I). Leur niveau de base est entre - I et - $2 \mathrm{~m}$ et leur destruction commence au niveau - I $\mathrm{m}^{82}$. Ils sont formés d'un ensemble cylindrique de briques crues divisé en deux parties par une sole: la partie inférieure était destinée au combustible, tandis que la partie haute à ciel ouvert servait de chambre de cuisson. Sous l'effet de la chaleur, les briques se sont vitrifiées. F. Bisson de La Roque affirma avoir remblayé et laissé en place ces fours pour en permettre l'étude par les générations futures ${ }^{83}$. Il faut cependant noter que nous ne savons pas comment se fit ce remblayage, ni s'il y a eu des fouilles dans la zone entre I930 et nos jours, ce qui a pu entraîner une destruction - au moins partielle - des fours. Quoi qu'il en soit, en raison des profondeurs des mesures géophysiques, tous les fours ne peuvent pas être détectés. C'est pourquoi nous avons sélectionné ceux qui se situent à une plus faible profondeur (points oranges sur la fig. 23). En outre, une inconnue subsiste quant au matériau utilisé pour leur comblement qui peut modifier la réponse attendue.

L'emplacement des trois fours sélectionnés est cohérent avec une zone d'anomalies dipolaires (marqués par un B dans la fig. 22) qui est un des critères marquant la vitrification des briques les constituant. Cependant, ils n'apparaissent ni comme anomalies de conductivité électrique, ni de susceptibilité magnétique comme a priori on s'y attendait (tabl. 2), ce qui peut s'expliquer par leur probable destruction partielle et leur comblement. De ce fait, la présence d'anomalies dipolaires sera par la suite considérée comme le premier critère de détection des fours, la conductivité électrique et la susceptibilité magnétique ne venant que confirmer cette information.

\footnotetext{
81 Recherche doctorale en cours. L'étude préliminaire (Barahona-Mendieta 20I4) avait déjà montré une production durant la XVIII ${ }^{e}$ dynastie, mais les prospections céramiques ont permis d'agrandir la fourchette chronologique pour la production céramique de ce secteur qui,

désormais, commence au moins à la XVII ${ }^{\mathrm{e}}$ dynastie.

82 Appelées «tours» par F. Bisson de La Roque: Bisson de La Roque i93I, p. 22.

83 «Les fours ont été en partie enterrés à nouveau afin d'essayer de les conserver et afin qu'une étude plus approfondie
}

puisse en être faite. Cette étude pourrait avantageusement comprendre une fouille plus étendue du niveau inférieur ou niveau entre -I mètre et - $2 \mathrm{~m}$. 47 ", Bisson de La Roque i93I, p. 25. 


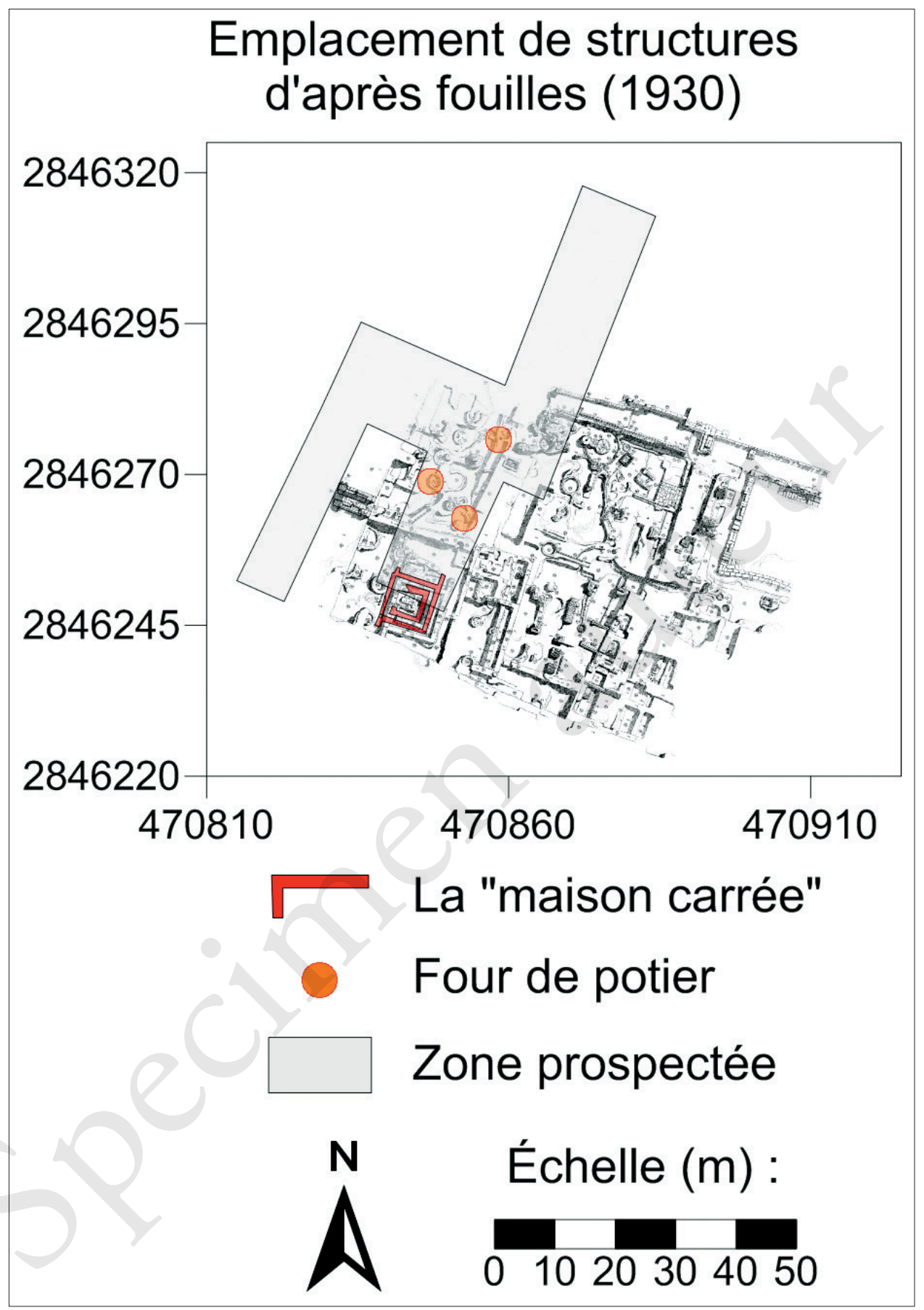

FIG. 23. Fours susceptibles d'être détectés par les appareils. 


\section{Le mur de clôture de $9 m$}

Le deuxième grand aménagement de la zone est un mur de clôture de $9 \mathrm{~m}$ d'épaisseur, orienté nord/sud, passant devant les kiosques et s'étendant vers le secteur sud-ouest. L'assemblage des plans (pl. I) a montré que son tracé n’est pas établi et nous avons voulu préciser nos connaissances par la prospection géophysique. Étant construit en briques crues, on s'attendrait à ce qu'il présente une faible susceptibilité magnétique et une forte conductivité électrique (tabl. 2).

En regardant les anomalies de conductivité électrique, on constate qu'une anomalie de forte valeur se trouve dans la partie est de la prospection et correspond à l'orientation indiquée sur le plan de 1930 ( $\mathrm{C}$ dans la fig. 22). Cette observation doit cependant être modérée par le fait qu'une zone de remontée de la nappe phréatique se trouve non loin (autour de la position E470860, N2846260, correspondant à l'anomalie de très forte conductivité électrique visible sur les trois cartes de la fig. 7). Une zone de faible susceptibilité magnétique présente un tracé similaire ( $\mathrm{C}$ dans la fig. 22), nous permettant de confirmer l'orientation globale du mur et sa correspondance avec le plan de I930. Il serait tentant d'identifier l'angle formé par cette zone de faible susceptibilité magnétique avec l'angle du mur de $9 \mathrm{~m}$ que F. Bisson de La Roque dit avoir découvert en 1930 et qui est visible pl. I, mais nous manquons d'éléments pour l'affirmer de manière certaine.

Afin de préciser l'identification correcte du mur, un nettoyage de surface a été effectué. Il a permis d'en dégager une partie à l'ouest des kiosques et de mesurer la conductivité électrique et la susceptibilité magnétique directement sur la structure (fig. 24). D'après les résultats obtenus, il apparaît que la courbe de conductivité électrique (en noir) est plus faible dans le remblai à l'extérieur du mur et augmente à l'intérieur du massif. En ce qui concerne la susceptibilité magnétique (en bleu), elle présente un résultat inverse, étant plus élevée dans le remblai et diminuant dans le mur, ce qui correspond aux résultats attendus pour les murs en briques crues $^{84}$. Cependant, la mesure de susceptibilité magnétique remonte de manière inhabituelle à $4,50 \mathrm{~m}$ à l'intérieur du massif et présente des résultats proches de ceux de la façade du mur. Il est possible que cela témoigne d'une exposition de cette partie à l'inondation comme s'il s'agissait d'une façade. Cela pourrait signifier que le mur n'aurait fait que 4,50 m d'épaisseur dans un premier temps et qu'il aurait été agrandi par la suite pour atteindre les $9 \mathrm{~m}$. Confirmer l'existence de deux étapes de construction et les dater dépasse le cadre de cet article. Signalons cependant que le mur ne peut être antérieur à Nectanébo I ${ }^{\text {er }}$ en raison de ses assises courbes ${ }^{85}$. Si l'élargissement du mur se confirme, il pourrait correspondre à une monumentalisation de l'entrée du temple.

84 BeCKer, FASSBINDER 1999.

85 La datation de cet aménagement pose de nombreux problèmes qui dépassent le cadre de cet article.
F. Bisson de La Roque l'attribuait au Nouvel Empire. Or, il semble d'après les photographies de fouilles que le mur soit à assises courbes. Cette question est largement traitée dans la thèse de F. Relats Montserrat (2016b). 


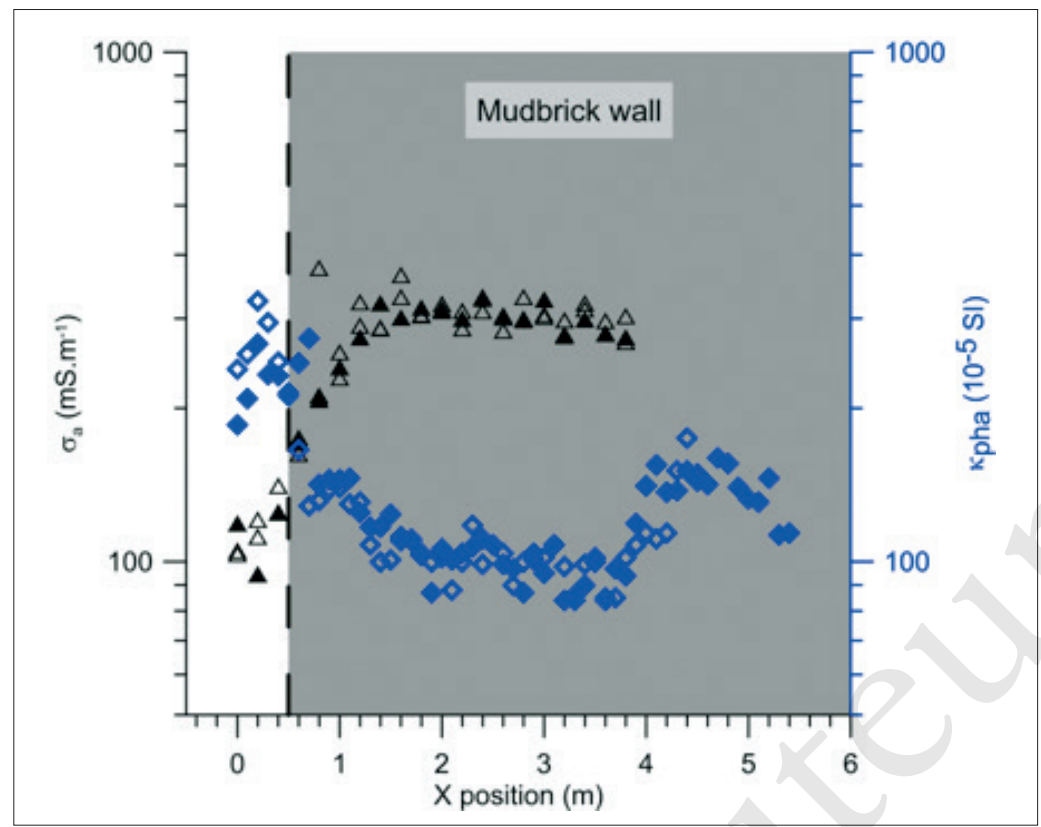

FIg. 24. Mesures de conductivité électrique et de susceptibilité dans le remblai et le mur de $9 \mathrm{~m}$.

\section{Le mur de clôture augustéen?}

À l'ouest du secteur s'étend le mur de clôture construit par Auguste dans lequel s'ouvre la porte de Tibère ${ }^{86}$. Son tracé entre la poterne et la porte de Tibère ne semble a priori pas poser problème puisque des briques sont encore présentes en surface. Il faut cependant signaler qu'il n'est pas clairement visible sur la fig. 22 (marqué par un D), alors qu'il devrait montrer une réponse similaire à celle du mur de $9 \mathrm{~m}$. Cela pourrait s'expliquer par la topographie du terrain, ainsi que par l'exposition des briques à l'air libre qui leur ferait perdre une partie de leurs propriétés. Néanmoins, cela change peu l'interprétation des vestiges qui sont visibles hors sol.

\section{La présence de la "maison carrée »?}

Les cartes de susceptibilité magnétique nous ont également permis de confirmer la présence potentielle d'un autre vestige: au sud-ouest, une anomalie positive de forme carrée au sein de laquelle se trouve une anomalie négative, elle aussi de forme carrée, se situe à l'endroit théorique des restes de la maison carrée (fig. 22 marquée par un E). D'après les fouilles de F. Bisson de La Roque en I930, I931 et $1932^{87}$, il s'agit d'un édifice en brique cuite de 8,50 m de côté dont aucun seuil ne fut découvert. Le niveau de base des murs est à $+0,88 \mathrm{~m}$, ces derniers subsistant sur une hauteur variant entre 0,25 et $0,48 \mathrm{~m}$ (niveau absolu : + I, I3 et + I,36 m). À l'intérieur de ce carré, se trouvait un autre carré d'environ $7 \mathrm{~m}$ de côté, séparé du carré extérieur par un espace de $0,80 \mathrm{~m}$. Comme Fl. Saragoza l'a récemment montré, il s'agit d'un sanctuaire

86 Revez 2004, avec la bibliographie des travaux de F. Bisson de La Roque.
87 Bisson de La Roque i93I, p. 7-9, fig. 4. ; Bisson de La RoQUe I933, p. 42. 
isiaque $^{88}$. Son plan devait originellement ressembler au sérapeion découvert dans le parvis du temple de Louqsor et qui occupe le même emplacement que la maison carrée de Médamoud par rapport au temple ${ }^{89}$. Nous ne pouvons expliquer la présence de zones de faibles valeurs qui s'étendent vers le nord de cette maison ${ }^{90}$.

Ainsi, même si nous n'avons pas pu superposer nos résultats aux plans des anciennes fouilles, nous pouvons assurer la présence des principaux vestiges qui avaient été découverts. Des zones d'ombre subsistent quant à leur tracé et leur état actuel, mais leur présence est assurée, ce qui permettra d'apporter des réponses aux questions laissées ouvertes.

\section{La zone centrale du kôm: zone $\mathrm{M}_{4} / \mathrm{K}_{9}$ (grande zone)}

La dernière zone présente une situation diamétralement différente aux deux précédentes. Elle se situe au centre du kôm, dans un secteur qui ne fut jamais fouillé, ni par F. Bisson de La Roque, ni par $\mathrm{Cl}$. Robichon et nous sommes donc dépourvus d'informations archéologiques pour interpréter les données géophysiques. Néanmoins, à la lumière des résultats obtenus dans les autres secteurs, nous avons pu mettre en place un protocole interprétatif nous permettant de proposer un certain nombre d'hypothèses. Pour ce faire, nous avons combiné de différentes manières les cartes d'interprétation de la zone (fig. 25) afin de faire ressortir les combinaisons qui nous paraissaient les plus significatives. La combinaison bleue (fig. 25a) est celle qui correspond le mieux aux structures pouvant être interprétées comme des fours. Une importance particulière a été accordée aux anomalies magnétiques (bleu uni plus foncé) car ce sont les seuls critères qui permettaient de reconnaître clairement les fours de la zone Pio/Ori. La combinaison verte (fig. 25b) permet de faire ressortir ce qui pourrait correspondre à des structures en brique cuite. Enfin, la combinaison rouge (fig. 25c) cherche à identifier les structures potentiellement en brique crue, en se basant sur les mesures prises directement sur le mur de $9 \mathrm{~m}$ et sur les prospections qui ont montré que les anomalies de forte conductivité électrique (rouge uni plus foncé) pouvaient correspondre aux briques crues. Les résultats de ces interprétations sont synthétisés dans la fig. 26.

88 Saragoza 20I2, p. 349-370.

89 Golvin et al. I98I, p. II8, fig. 2 e. 90 Visibles sur la fig. 22. Une zone de faible valeurs prolonge la maison carrée et correspond à l'orientation d'une canalisation signalée par F. Bisson de La Roque sur le plan de I930 (Bisson de La RoQue i93I, pl. I).
La zone est cependant bien plus étendue et ne peut donc pas être identifiée à la canalisation. 


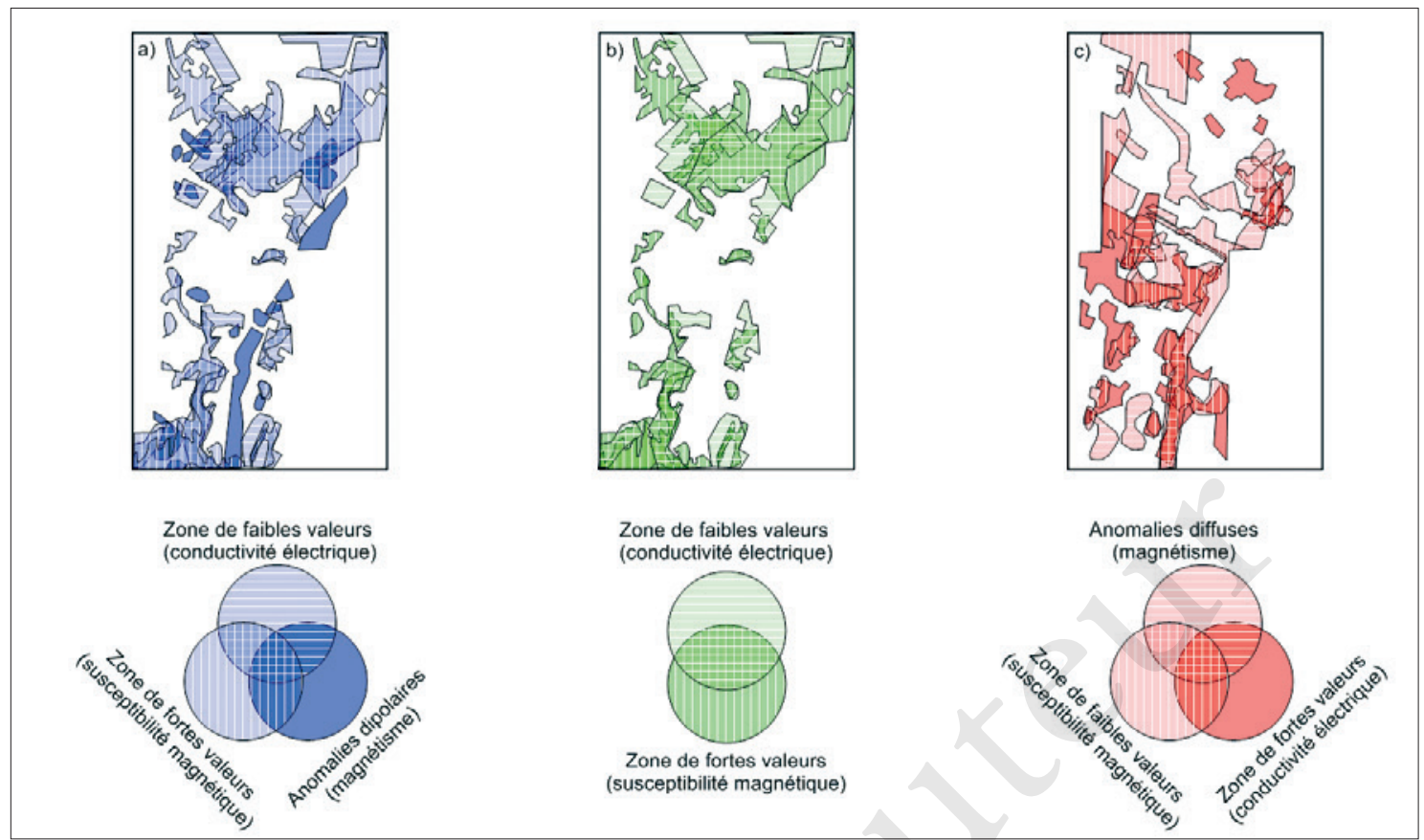

Fig. 25. Synthèse globale des données géophysiques.
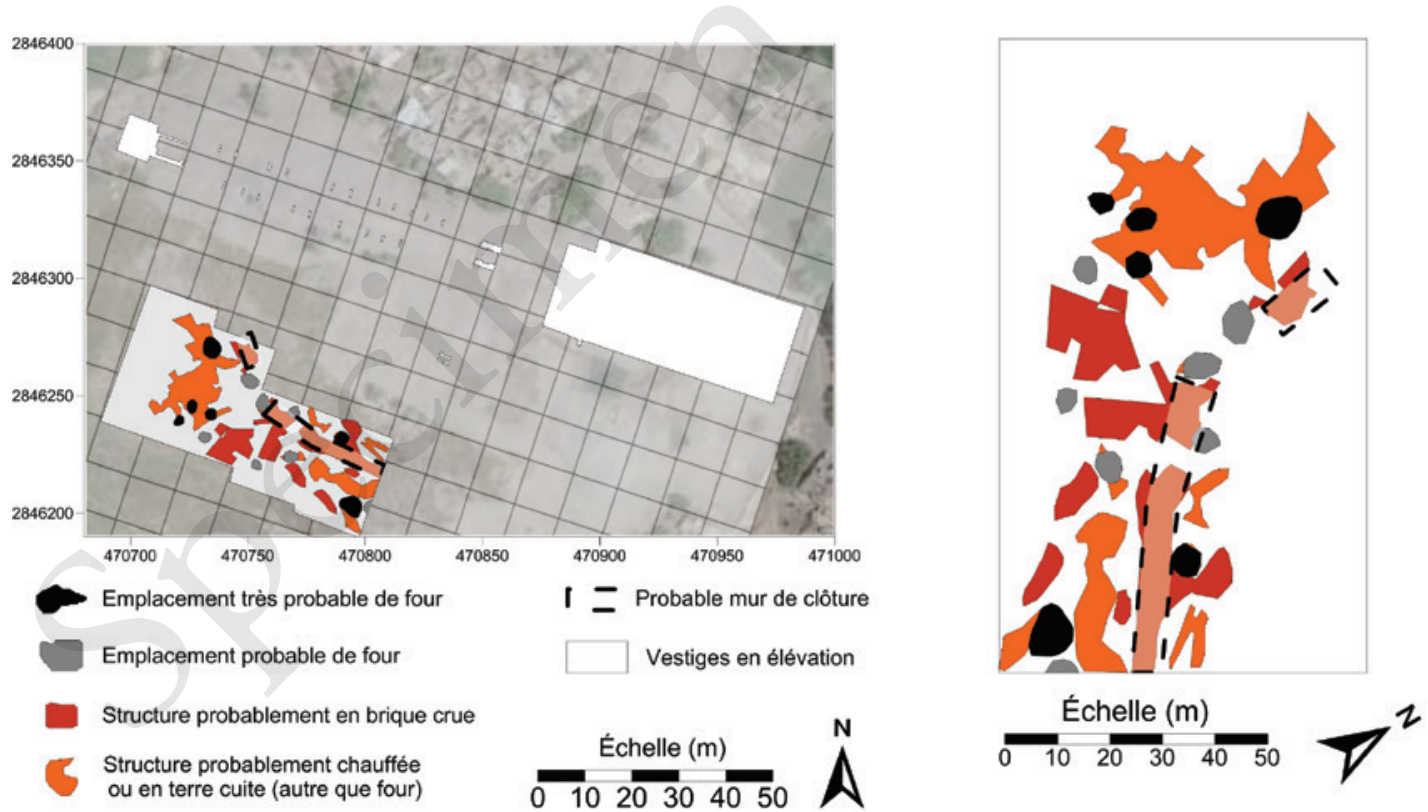

Fig. 26. Hypothèses d'interprétation archéologique tirées de la fig. 25. 


\section{La présence certaine de fours: un troisième secteur de production céramique à Médamoud}

La prospection céramique a établi que cette zone a gardé les traces d'un important quartier d'activité de potiers: la densité des tessons, ainsi que les déchets de cuisson, associés à des scories, sont des indices clairs suggérant la présence d'ateliers. L'argument le plus probant est, rappelons-le, la paroi de four trouvée à même le sol hors contexte (fig. I6). La datation des céramiques couvre une grande fourchette, de la XVIII ${ }^{\mathrm{e}}$ dynastie à l'époque ptolémaïque, avec des déchets de production à toutes les époques. Au sein de cette documentation, nous devons mettre en exergue l'abondance de fragments de jarres de stockage (Meat Jars) bien connues pendant la deuxième moitié de la XVIII ${ }^{\mathrm{e}}$ dynastie et les $\mathrm{XIX}^{\mathrm{e}} / \mathrm{XX}^{\mathrm{e}}$ dynasties $^{9 \mathrm{I}}$. On notera également la grande présence d'imitations d'amphores égéennes d'époque hellénistique. Bien sûr, nous ne pouvons pas assurer que l'ensemble de la production vient de cette zone, mais les résultats géophysiques pourraient plaider en faveur de la présence d'ateliers. Ainsi, en reprenant les trois critères précédemment établis (anomalie magnétique dipolaire, faible conductivité électrique, forte susceptibilité magnétique), au moins I 3 combinaisons d'anomalies pourraient correspondre à des structures de type fours (fig. 26 - interprétations noires et grises). Si nous comparons l'emplacement supposé de ces fours, il est probable que nous ayons plusieurs phases de fours qui se seraient succédé. En ce sens, plusieurs fours sont situés à proximité (peut-être partiellement recouverts?) par un mur de clôture dont nous reparlerons, ce qui est un indice des réaménagements du secteur.

Seule une fouille nous permettra, dans les prochaines années, de confirmer ces résultats. À l'échelle du site, il y aurait donc eu trois pôles de production: le premier au sud-ouest du temple, à la fin de la Deuxième Période intermédiaire et au début du Nouvel Empire; le deuxième au sud-ouest du kôm dès la fin de la Troisième Période intermédiaire à l'époque ptolémaïque $\left(\mathrm{G}_{7} / \mathrm{C}_{\mathrm{IO}}\right)$; et un troisième dans la grande zone $\left(\mathrm{M}_{4} / \mathrm{K}_{9}\right)$, qui présente une chronologie intermédiaire. On peut donc poser l'hypothèse d'un glissement progressif vers le sud-ouest des zones de production qui s'éloignent progressivement du temple. La présence de tessons de la XVIII dynastie et de l'époque ptolémaïque pourrait être le signe de dépotoirs de déchets d'autres secteurs.

\section{Un nouveau mur de clôture?}

En comparant les cartes d'anomalies et de susceptibilité magnétiques, une structure orientée nord-ouest/sud-est est clairement visible. Sa présence est confirmée du fait qu'elle apparaît à toutes les profondeurs (fig. 25). Il pourrait s'agir d'un mur de briques crues d'approximativement 4,5 m d'épaisseur (fig. 26). Une ouverture apparaît en négatif dans le mur par l'arrêt des briques sur une surface de $15 \mathrm{~m}$. À cet emplacement, la faible réponse en susceptibilité magnétique semble dessiner une structure bâtie (fig. II) qui répond de la même façon que le dallage entre la porte de Tibère et les kiosques. Peut-être aurions-nous ici une porte en pierre formant une avancée dans le mur. Il faudra bien évidemment étendre la zone de prospection pour comprendre la relation qu'un tel dispositif pouvait entretenir avec les murs de clôture du téménos, d'autant que nous ne pouvons le dater. Mais il semble qu'il recouvre partiellement plusieurs fours. Il leur est ainsi postérieur. 
Le reste de la zone est marqué par un enchevêtrement de structures qui en empêchent une lecture claire. Plusieurs phases de constructions peuvent néanmoins être détectées. Celles-ci se superposent puisque l'orientation des vestiges est différente selon les profondeurs. En outre, les réponses correspondent aussi bien aux résultats attendus pour des structures en brique crue (fig. 25c) qu'en brique cuite (fig. 25b), ce qui est probablement le signe de la stratification complexe du secteur. Nous serions tentés d'y voir - au moins dans une des phases d'occupation - un quartier artisanal, s'il se confirme qu'une partie de ces structures entretient un rapport avec les fours situés à proximité. Seule la fouille apportera une réponse quant à l'usage de ce secteur à l'extérieur du mur de clôture augustéen et au lien qu'il entretenait avec lui.

\section{Conclusion}

Les résultats que nous avons présentés ne sont que les prémices de nouvelles activités sur le terrain, mais ils nous permettent déjà d'améliorer notre connaissance du site. La présence des déchets de coction assure que Médamoud était un des principaux centres de production de céramique à pâte calcaire entre la Deuxième Période intermédiaire et l'époque romaine, sans interruption majeure. Nous connaissions déjà deux zones de fours et la prospection géophysique offre de fortes présomptions sur l'existence d'autres zones, en particulier un quartier artisanal et un nouveau mur de clôture. Les résultats dans les abords du temple sont plus limités, mais confirment la présence d'une partie des vestiges découverts par F. Bisson de La Roque.

Dans les prochaines campagnes, la prospection sera étendue à la totalité du kôm et nous ouvrirons de nouveaux secteurs de fouilles pour préciser les résultats obtenus.

\section{ANNEXE: MÉTHODOLOGIE POUR LE TRACÉ DES FIGURES D'INTERPRÉTATION DES CARTES DE PROPRIÉTÉS APPARENTES}

L'objet de cette annexe est de décrire les étapes permettant d'obtenir des cartes d'interprétation. Nous prendrons pour cela l'exemple de l'interprétation des données de susceptibilité magnétique obtenue sur la zone $\mathrm{M}_{4} \mathrm{~K}_{9}$ (fig. Io et fig. II). Nous partons du postulat que la distribution statistique des données soit une loi normale. Le modèle associé permet alors de définir des estimateurs statistiques (comme la moyenne, l'écart-type, la médiane ou des quartiles). À partir de ceux-ci on peut déduire les intervalles suivants:

- l'intervalle de deux écart-types centré sur la moyenne;

- l'intervalle compris entre le premier quartile (valeur du paramètre qui fait que $25 \%$ des valeurs lui soient inférieures) et le troisième quartile (valeur du paramètre qui fait que $75 \%$ des valeurs lui soient inférieures).

Le premier intervalle défini contient ainsi près de $68 \%$ des valeurs prises par la propriété mesurée. Le second, quant à lui, contient par définition $50 \%$ des valeurs. La moyenne et la médiane d'une loi normale étant égales (en pratique assez proches), il est alors possible de faire une échelle de couleurs qui se base sur les deux intervalles décrits ci-dessus et qui permet 
notamment d'identifier des pseudo-contours ne contenant que I4 \% des valeurs données se situant entre la valeur de la moyenne retranchée d'un écart-type et le premier quartile (valeurs limites entre faibles et intermédiaires) d'une part, et les valeurs comprises entre le troisième quartile et la valeur de la moyenne ajoutée d'un écart-type (valeurs limites entre intermédiaires et fortes) d'autre part.

\begin{tabular}{|c|c|c|c|c|c|c|c|}
\hline minimum & $\begin{array}{c}\text { Moyenne - } \\
\text { écart-type }\end{array}$ & $\begin{array}{c}\text { Premier } \\
\text { quartile }\end{array}$ & Moyenne & médiane & $\begin{array}{c}\text { Troisième } \\
\text { quartile }\end{array}$ & $\begin{array}{c}\text { Moyenne } \\
\text { + écart-type }\end{array}$ & maximum \\
\hline I49,5 & 295,5 & 314,5 & 358,5 & 355 & 398,5 & 422 & $6 \mathrm{I} 5$ \\
\hline
\end{tabular}

TABL. 8: Élément de statistiques basique pour la prospection de susceptibilité magnétique de la voie 2 sur $\mathrm{M}_{4} \mathrm{~K}_{9}$.

Une fois que l'on définit ces seuils, on peut représenter les valeurs sous forme d'histogramme (fig. 27). Cette représentation permet de voir que les intervalles de transition sélectionnés sont assez fins vis-à-vis de la distribution totale. L'intervalle compris entre les deux intervalles de transition est marqué en vert pour faciliter la lecture (fig. 27). Lopération est réalisée pour chaque prospection d'un même paramètre recouvrant la même surface. On superpose ensuite les cartes obtenues. Les contours les plus marqués sur la superposition sont alors ceux qui sont pointés manuellement (fig. 28).

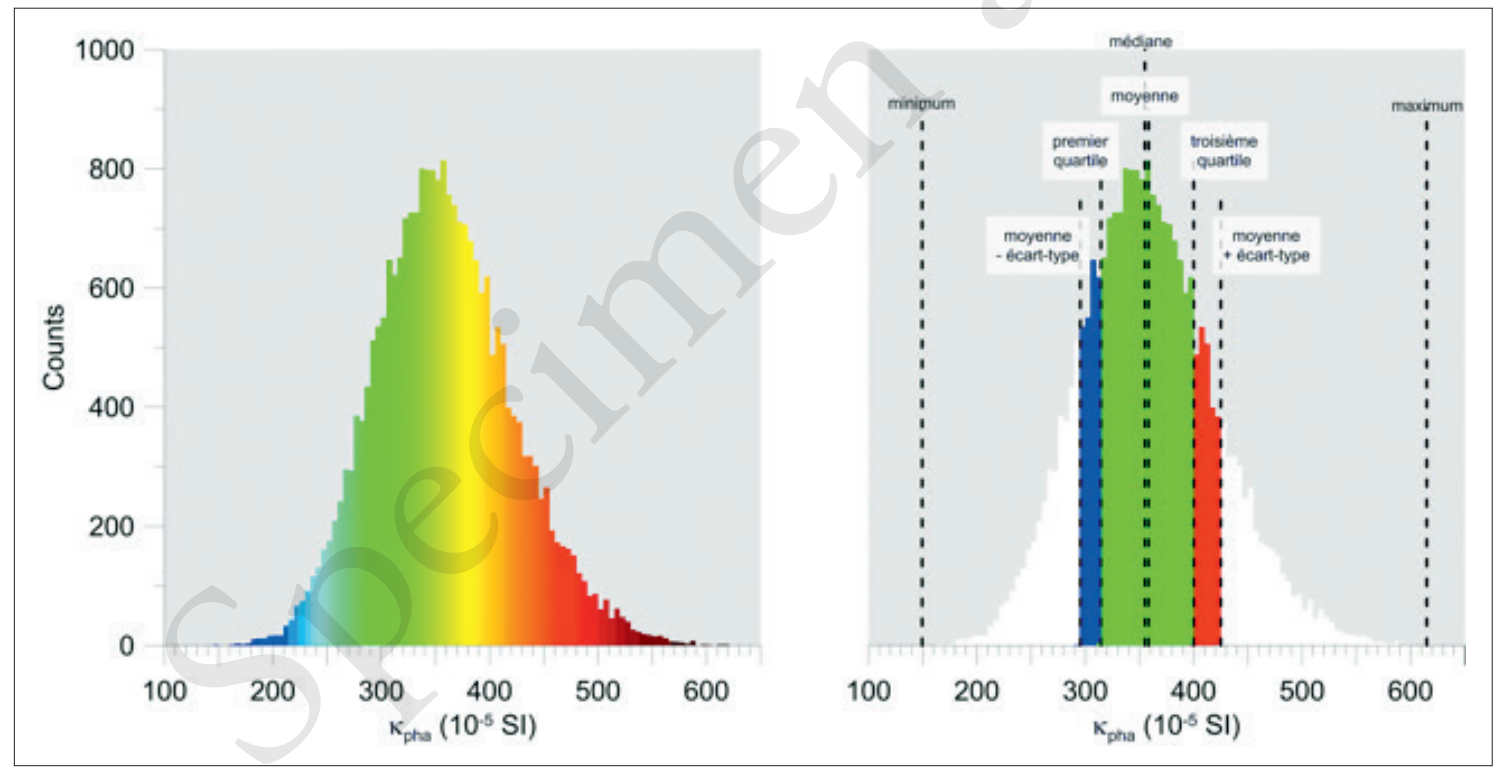

Fig. 27. Histogramme de la représentation en échelle de couleur continue (à gauche de la figure) et en échelle d'aide à l'interprétation (à droite de la figure). 


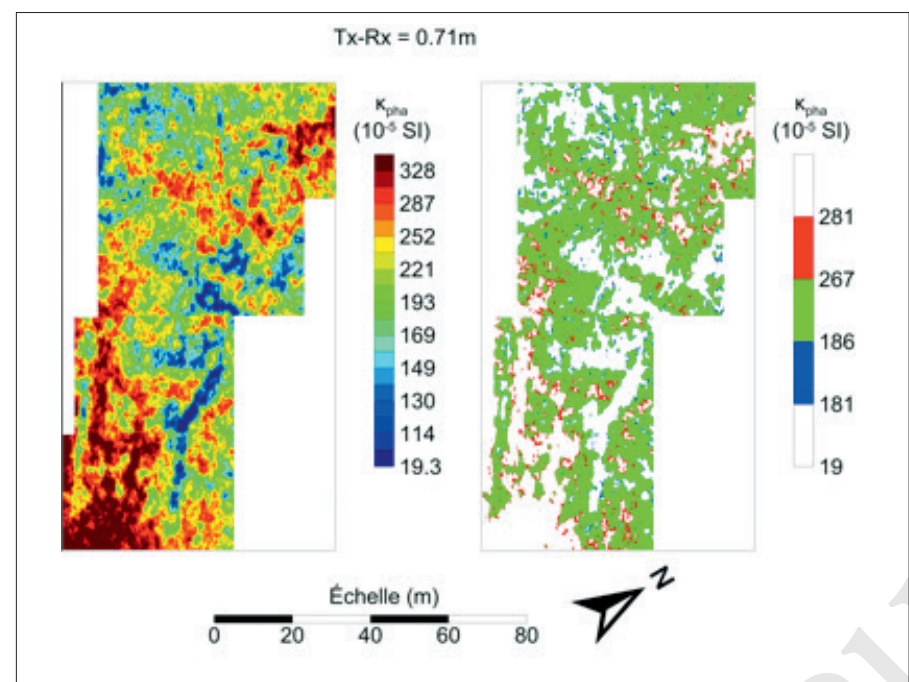

Fig. 28. Prospection de la susceptibilité magnétique sur la zone $\mathrm{M}_{4} \mathrm{~K}_{9}$, carte obtenue avec les échelles de la fig. 27.

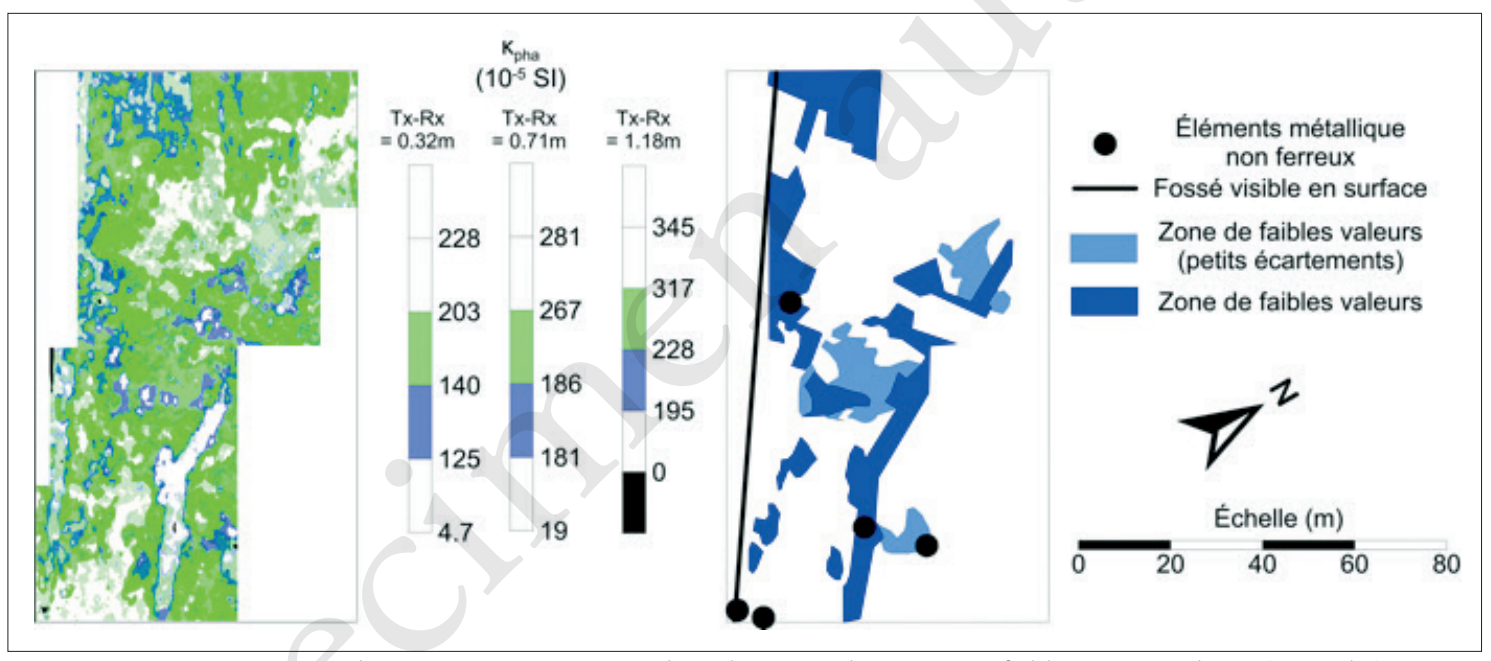

Fig. 29. Carte superposée des 3 voies avec mise en valeur des zones de transition faible à intermédiaire (à gauche) et résultats du pointé manuel (à droite).

Quelques remarques sont nécessaires sur les limites de la méthode. L'hypothèse de normalité de la distribution des valeurs n'est pas forcément respectée, en particulier s'il y a des contrastes de propriété bien marqués avec des répartitions spatiales inégales. Cependant, dans ce cas-là, l'interprétation est, a priori, plus simple et ne nécessite pas de mise en valeur pour le pointé. Le fait d'évaluer des seuils de façon statistique gomme une partie de l'information de la variation des propriétés avec l'écartement (ce qui peut être une piste d'amélioration). 


\section{BIBLIOGRAPHIE}

Abdallatif, Mousa, Elbassiony 2003

T.F. Abdallatif, S.E. Mousa, A. Elbassiony, «Geophysical Investigation for Mapping the Archaeological Features at Qantir, Sharqyia, Egypt», Archaeological Prospection 6, vol. Io, 2003, p. 27-42.

Aston 1998

D.A. Aston, Die Keramik des Grabungplatzes Q I. Teil I, Corpus of Fabrics, Wares and Shapes, Mayence, 1998.

Aston 1999

D.A. Aston, Elephantine XIX, Pottery from the Late New Kingdom to the Early Ptolemaic Period, ArchVer 95, Mayence, I999.

Aston 2004

D.A. Aston, Tell el-Dab'a XII. A Corpus of Late Middle Kingdom and Second Intermediate Period Pottery, UZK 23, DÖAWW 28, Vienne, 2004.

Aston 2007

D. Aston, «Kom Rabia, Ezbet Helmi and Saqqara NK3507. A Study in Cross-Dating» in M. Bietak (éd.) The Synchronisation of Civilisations in the Eastern Mediterranean in the Second Millenium B.C. III, Österreichische Akademie der Wissenschaften, Vienne, 2007, p. 207-248.

Aston, BADER 2009

D.A. Aston, B. Bader, «Fishes, Ringstands, Nudes and Hippos - A Preliminary Report on the Hyksos Palace Pit Complex L8I", ÄgLev i9, 2009, p. 19-89.

BAILEY 1988

D.M. Bailey, A Catalogue of the Lamps in the British Museum, vol. 3, Roman Provincial Lamps, British Museum, Londres, I988

BALLET et al. I99I

P. Ballet, F. Mahmoud, M. Vichy, M. Picon, «Artisanat de la céramique dans l'Égypte romaine tardive et byzantine. Prospections d'ateliers de potiers de Minia à Assouan", CCE 2, I99I, p. I29-I44.

\section{BALLET 2OOI}

P. Ballet, «Céramiques hellénistiques et romaines d'Égypte» in É. Gény (éd.), Céramiques hellénistiques et romaines III, Paris, 2008.

Barahona-Mendieta 2014

Z. Barahona-Mendieta, «La producción cerámica en Medamud. Estudio de la cerámica procedente de los hornos del Reino Nuevo, Baja Época y Época Ptolemaica», $B C E$ 24, 2014, p. 267-280.

Barahona-Mendieta 2015

Z. Barahona Mendieta, «Note préliminaire sur quelques céramiques grecques trouvées à Médamoud» in A. Marangou (éd.), Table ronde: La culture matérielle grecque dans la vallée thébaine. 8 novembre 20I2, université Rennes 2, 20I3, Rennes, 20I5, p. II3-II7.

Barahona-Mendieta 2016

Z. Barahona-Mendieta, Estudio histórico y caracterización diacrónica de la cerámica descubierta en Medamud en las excavaciones del IFAO: contribución a la historia de la cerámica del Alto Egipto, thèse de doctorat, Universitat Autonoma de Barcelona, 2016.

Barahona-Mendieta 2017

Z. Barahona-Mendieta, «La producción cerámica en época ptolemaica en Medamud, hasta comienzos de la dominación romana" in R. David (éd.), Céramiques ptolémaïques de la région thébaine, CCE Io, Le Caire, 20I7, p. 25-48.

Barahona-Mendieta, Relats Montserrat à paraître

Z. Barahona-Mendieta, F. Relats Montserrat, «Medamud en el Tercer Periodo Intermedio y en la Baja Época. Consideraciones preliminares» in A. Largacha (éd.), V Congreso Ibérico de Egiptología, Cuenca 9-I2 mars 2015, 2017, p. I27-I47. 
BeCKer, FASSBINDER I999

H. Becker, J.W.E. Fassbinder, "In Search for Piramesse- the Lost Capital of Ramses II in the Nile Delta (Egypt) by Caesium Magnetometry", Archaeological Prospection 6, vol. 4, 1999, p. I46-I5O.

Bisson de La RoQue 1926

F. Bisson de La Roque, Rapport sur les fouilles de Médamoud (années 1924-1925), FIFAO 3, vol. I, Le Caire, 1926.

Bisson de La Roque 1927

F. Bisson de La Roque, Rapport sur les fouilles de Médamoud (1926), FIFAO 4, vol. I, Le Caire, I927.

Bisson de La RoQue 1930

F. Bisson de La Roque, Rapport sur les fouilles de Médamoud (année I929), FIFAO 7, vol. I, Le Caire, 1930.

Bisson de La Roque i93I

F. Bisson de La Roque, Rapport sur les fouilles de Médamoud (I930), FIFAO 8, vol. I, Le Caire, I93I.

Bisson de La Roque 1933

F. Bisson de La Roque, Rapport sur les fouilles de Médamoud (I93I et 1932), FIFAO 9, vol. 3, Le Caire, 1933.

Bisson de La Roque 1946

F. Bisson de La Roque, «Les fouilles de l'Institut français à Médamoud ", $R d E$ 5, 1946, p. 25-44.

Bisson de la Roque, Clère 1929

F. Bisson de La Roque, J.J. Clère, Rapport sur les fouilles de Médamoud (1928), FIFAO 6, vol. I, Le Caire, 1929.

Bisson de la Roque, Clère, Drioton 1928

F. Bisson de La Roque, J.J. Clère, Ét. Drioton, Rapport sur les fouilles de Médamoud (I927), FIFAO 5, vol. I, Le Caire, 1928.

BONIFAY 2004

M. Bonifay, Études sur la céramique romaine tardive d'Afrique, BAR-IS I301, Oxford, 2004.

BOURRIAU I990

J. Bourriau, "The Pottery» in P. Lacovara, Deir el-Ballas, Preliminary Report on the Deir el-Ballas Expedition, 1980-1986, ARCE Reports I2, Winona Lake, p. 15-65.
BOURRIAU 1997

J. Bourriau, «Beyond Avaris: The Second Intermediate Period in Egypt Outside the Eastern Delta" in E. Oren, (éd.), The Hyksos: New Historical and Archaeological Perspectives. International Seminar on Cultural Interconnections in the Ancient Near East, University Museum Monograph 96, Philadelphie, I997, p. I59-I82.

BOURRIAU 2010

J. Bourriau, The Survey of Memphis IV. Kom Rabia: The New Kingdom Pottery, EES ExcMem 93, Londres, 2010.

Bourriau, Op de Beek, Meyer 2005

J. Bourriau, L. Op de Beeck, M. de Meyer, "The Second Intermediate Period and Early New Kingdom at Deir al-Barsha", ÄgLev I5, 2005, p. IOI-I3O.

BRUNTON 1930

G. Brunton, Qau and Badari III, BSAE 5o, Londres, I930.

BUDKA 2006

J. Budka, "The Oriental Institute Ahmose and Tetisheri Project at Abydos 2002-2004: The New Kingdom Pottery", ÄgLev I6, 2006, p. 83-120.

Cabrol 200I

A. Cabrol, Les voies processionnelles de Thèbes, OLA 97, Louvain, 200 I.

DAVID 2013

R. David, «La céramique d'un habitat du v siècle à Karnak», Cahiers de Karnak I4, 2013, p. 287-297.

Defernez, Marchand 2006

C. Defernez, S. Marchand, «Imitations égyptiennes de conteneurs d'origine égéenne et Levantine (vi ${ }^{\mathrm{e}}$ s.-II ${ }^{\mathrm{e}}$ s. av. J.-C.) " in B. Mathieu, D. Meeks, M. Wissa (éd.), L'apport de l'Égypte à l'histoire des techniques. Méthodes, chronologie et comparaisons, BiEtud I42, Le Caire, 2006, p. 63-99.

Dixneuf 2OII

D. Dixneuf, Amphores égyptiennes. Production, typologie, contenu et diffusion (III siècle avant J.-C. - IX siècle après J.-C.), EtudAlex 22, Alexandrie, $2 \mathrm{OII}$ 


\section{GABOLDE 1998}

L. Gabolde, Le "grand château d'Amon» de Sésostris Ier $^{e}$ à Karnak, MAIBL 17 , Paris, 1998

Gabolde, Rondot 1993

L. Gabolde, V. Rondot, «Une catastrophe antique dans le temple de Montou à Karnak-Nord», BIFAO 93, 1993, p. 245-64.

GEMPELER 1992

R.D. Gempeler, Elephantine Io. Die Keramik römischer bis früharabischer Zeit, AV 43, Mainz, 1992.

Golvin et al. 198I.

J.-Cl. Golvin, S. 'Abd el-Hamīd, G. Wagner, Fr. Dunand, "Le petit Sarapieion romain de Louqsor», BIFAO 8I, I98I, p. II5-I48.

JACQUET-GORDON I98I

H. Jacquet-Gordon, "A Tentative Typology of Egyptian Bread Moulds» in Do. Arnold (éd.), Studien zur Altägyptischen Keramik, SDAIK 9, Mayence, p. II-24

JACQUET-GORDON 2012

H. Jacquet-Gordon, Karnak-Nord X, Le Trésor de Thoutmosis I $^{\text {r }}$ La céramique, FIFAO 65, Le Caire, 2012.

KOPETZKY 2012

K. Kopetzky, «The Dawn of the Middle Kingdom at Tell el-Dabca: Selected Pottery from Settlements and Tombs from the Phases G/I-3 and F" in R. Schiestl, A. Seiler (éd.), Middle Kingdom Pottery Handbook, vol. II, Vienna, 20I2, p. 89-Io6.

LAUFFraY I97I

J. Lauffray, «Abords occidentaux du premier pylône de Karnak, le dromos, la tribune et les aménagements portuaires», Kêmi 2I, I97I, p. 77-I44.

LAUFFRAY 1995

J. Lauffray, La chapelle d'Achôris à Karnak I. Les fouilles, l'architecture, le mobilier et l'anastylose, Paris, 1995 .

\section{MarChand 2012}

S. Marchand, "La céramique» in G. Charloux, R. Angevin, S. Marchand, H. Monchot, J. Roberson, H. Virenque, Le parvis du temple d'Opet à Karnak. Exploration archéologique (2006-2007), BiGen 4I, Le Caire, 20I2, p. 69-I24.
Marchand, Soukiassian 2010

S. Marchand, G. Soukiassian, Balat VIII. Un habitat de la XIII dynastie-2 ${ }^{e}$ Période intermédiaire

à Ayn Asil, FIFAO 59, Le Caire, 2010.

MASSON 2007

A. Masson, «Le quartier des prêtres du temple de Karnak: rapport préliminaire de la fouille de la maison VII, 200I-2003", Cahiers de Karnak I2, 2007, p. 593-655.

Masson $201 \mathrm{I}$

A. Masson, «Persian and Ptolemaic Ceramics from Karnak: Change and Continuity ", CCE 9, 20II, p. 269-310.

NordStröm, BourriaU 1993

H. Nordström, J. Bourriau, «Ceramic Technology: Clays and Fabrics» in Do. Arnold, J. Bourriau (éd.), An Introduction to Ancient Egyptian Pottery, SDAIK 17, Mayence, 1993 p. I42-190.

Pierrat et al. 1995

G. Pierrat, J.-P. Adam, Chr. Barbotin, M. Étienne, S. Guichard, D. Leconte, «Fouilles du musée du Louvre à Tod, I988-I99I ", CahKarn Io, I995, p. 405-503.

Pierrat-Bonnefois 1996

G. Pierrat-Bonnefois, «Évolution de la céramique de Tôd. Du II ${ }^{\mathrm{e}}$ siècle au viI ${ }^{\mathrm{e}}$ siècle apr. J.-C.", CCE 4, 1996, p. 189-206.

Pillet 1924

M. Pillet, «Rapport sur les travaux de Karnak (I923-I924)", $A S A E$ 24, I924, p. 5I-88.

Pillet 1925

M. Pillet, «Rapport sur les travaux de Karnak (19241925)", $A S A E$ 25, 1925, P. I-24.

Relats Montserrat 20i6a

F. Relats Montserrat, "Medamoud and the Nile: Some Preliminary Reflections» in H. Willems (éd.), The Nile. A Natural and a Cultural Landscape, Mainzer Historische Kulturwissenschaften, Mayence, 2016. 
Relats Montserrat 20i6b

F. Relats Montserrat, Les fouilles françaises de Médamoud, synthèse historique et archéologique d'un temple thébain, thèse de doctorat, université Paris-Sorbonne, 2016.

ReVEZ 2004

J. Revez, «Une stèle commémorant la construction par l'empereur Auguste du mur d'enceinte du temple de Montou-Rê à Médamoud", BIFAO I04, 2004, p. 495-510

RoBICHON, VARILLE 1939

Cl. Robichon, A. Varille, "Médamoud, les fouilles du musée du Louvre, 1938 ", $C d E$ I4, fasc. 27, janvier 1939, p. 82-7.

Robichon, VariLLE 1940

Cl. Robichon, A. Varille, Description sommaire du temple primitif de Médamoud, RAPH II, Le Caire, I940.

RodZIEWICZ 2005

M. Rodziewicz, Elephantine XXVII. Early Roman Industries on Elephantine, ArchVer I07, Berlin, Mayence, 2005.

RZEUSKa 2OII

T.I. Rzeuska, «Grain, Water and Wine. Remarks on the Marl A3 Transport-Storage Jars from Middle Kingdom Elephantine», $C C E$ 9, 20II, p. 46I-530.

SARAGOZA 2012

Fl. Saragoza, "La «maison à double-carré» de Médamoud et les sanctuaire isiaques d'Égypte», BIFAO II2, 20I2, p. 349-70.

SCHREIBER 2003

G. Schreiber, Late Dynastic and Ptolemaic Painted Pottery from Thebes (4th-2th c. BC), Dissertationes Pannonicae Ser. III. vol. 6, Budapest, 2003.

SeIdLmayer 200I

St.J. Seidlmayer, Historische und moderne Nilstände Untersuchungen zu den Pegelablesungen des Nils von der Frühzeit bis in die Gegenwart, ACHET I, Berlin, 20oI.
SEILER I997

A. Seiler, «Hebua I. Second Intermidiate Period and Early New Kingdom Pottery", CCE 5, 1997, p. 23-33.

SEILER 1999

A. Seiler, "Zur Formentwicklung der Keramik der Zweiten Zwischenzeit und der frühen 18. Dynastie» in W. Kaiser et al., «Stadt und Tempel von Elephantine, 25./26./27. Grabungsbericht», MDAIK 55, 1999, p. 204-224.

SCHIESTL, SEILER 2012

R. Schiestl, A. Seiler (éd.), Handbook of the Pottery of the Egyptian Middle Kingdom, 2 vol., ÖAW 72, CCEM 3I, Vienne, 2012.

TOMBER 2006

R.S. Tomber, "The Pottery" in V.A. Maxfield, D.P.S. Peacock (éd.), Survey and Excavation Mons Claudianus 1987-1993, vol. III, Ceramic Vessels and Related Objects, FIFAO 54, Le Caire, 2006, p. 3-2I8.

Tyson-SMITH 2012

S. Tyson-Smith, «Pottery from Askut and Nubian Forts" in R. Schiestl, A. Seiler (éd.), Handbook of the Pottery of the Egyptian Middle Kingdom, vol. II, Vienne, p. 377-405.

TRAUNECKER I97I

Cl. Traunecker, «Données d'hydrologie et de climatologie du site de Karnak (II) ", Kémi 2I, I97I, p. I77-I96.

TRAuneCKer I972

Cl. Traunecker, «Les rites de l'eau à Karnak d'après les textes de la rampe de Taharqa", BIFAO 72, I972, p. 195-236.

VARILLE 1943

A. Varille, Karnak Nord I, FIFAO I9, Le Caire, I943 


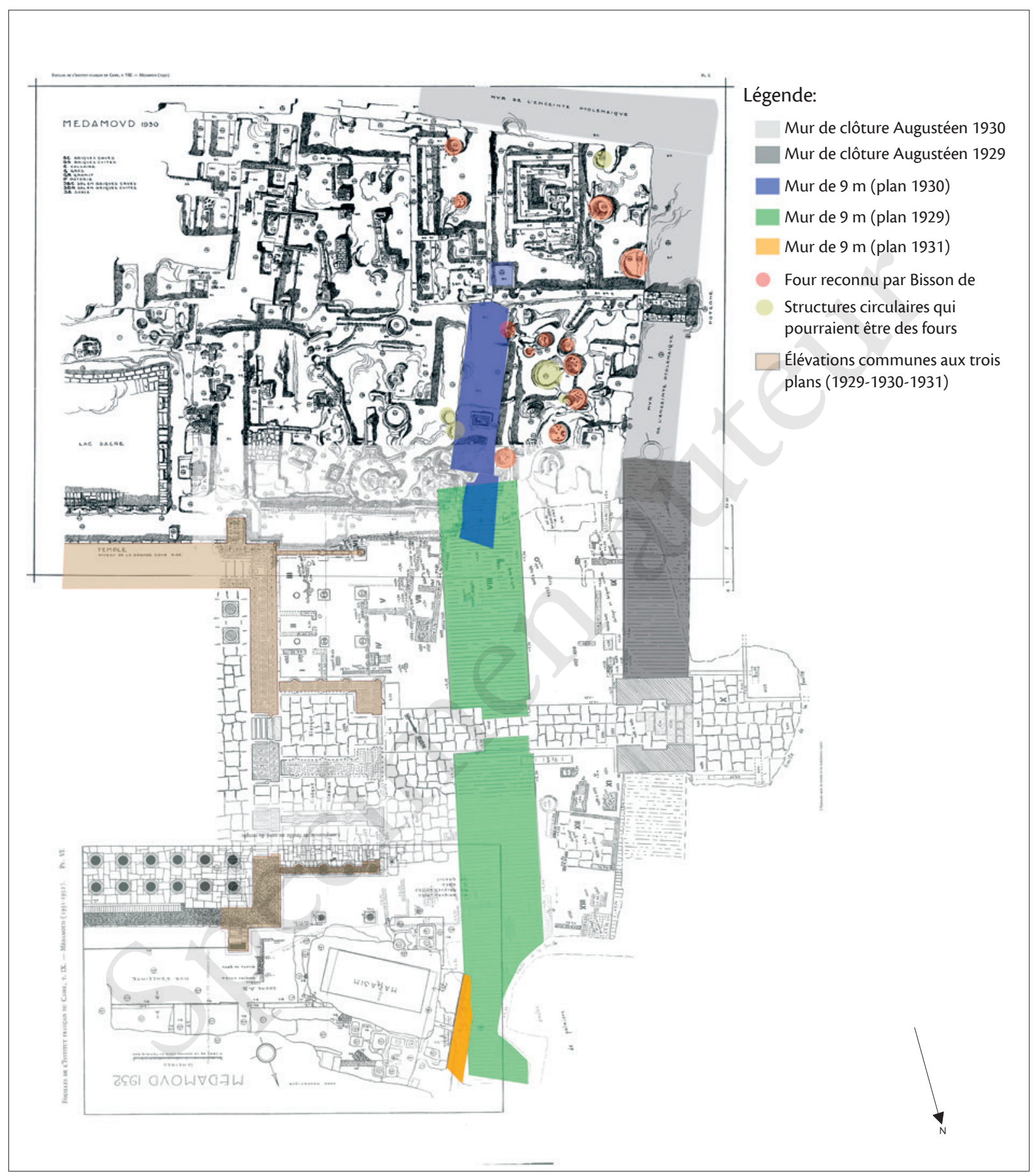

PL. I. Superposition des plans. 


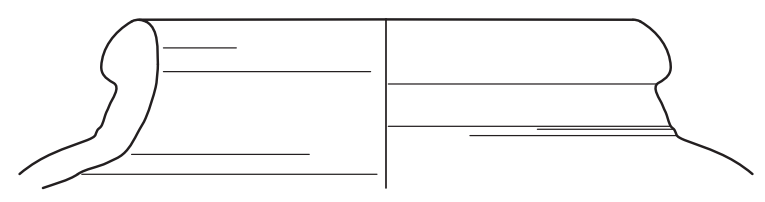

Med 2015-161

Marl A3
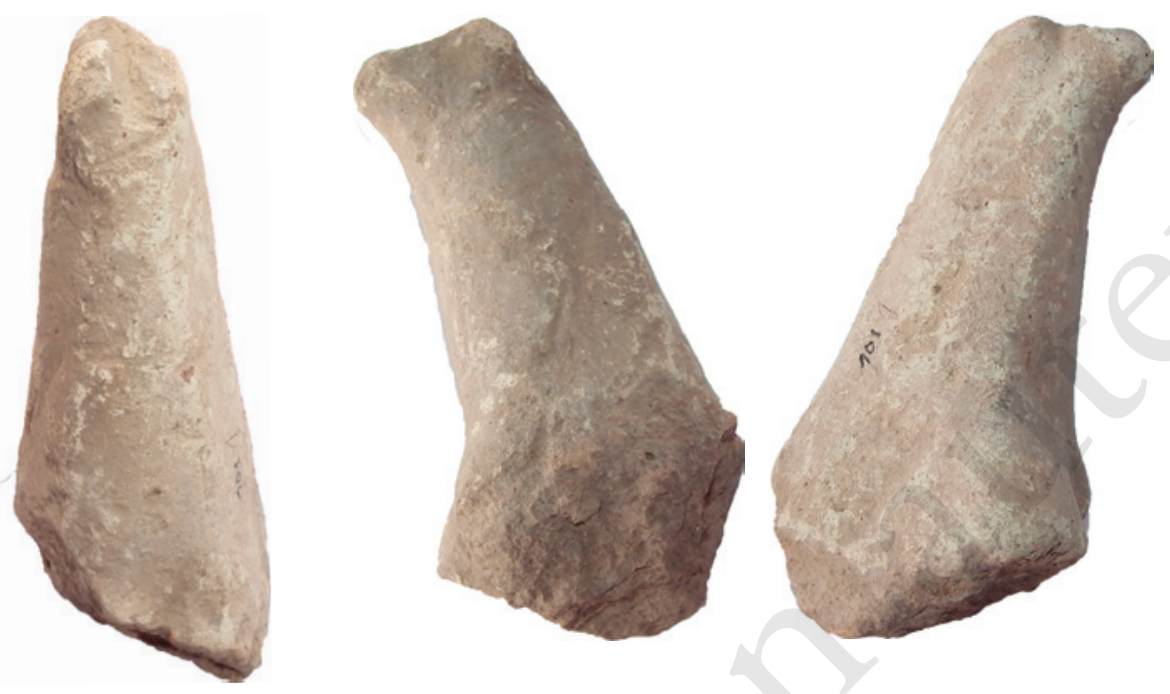

Med15-101

Marl A3
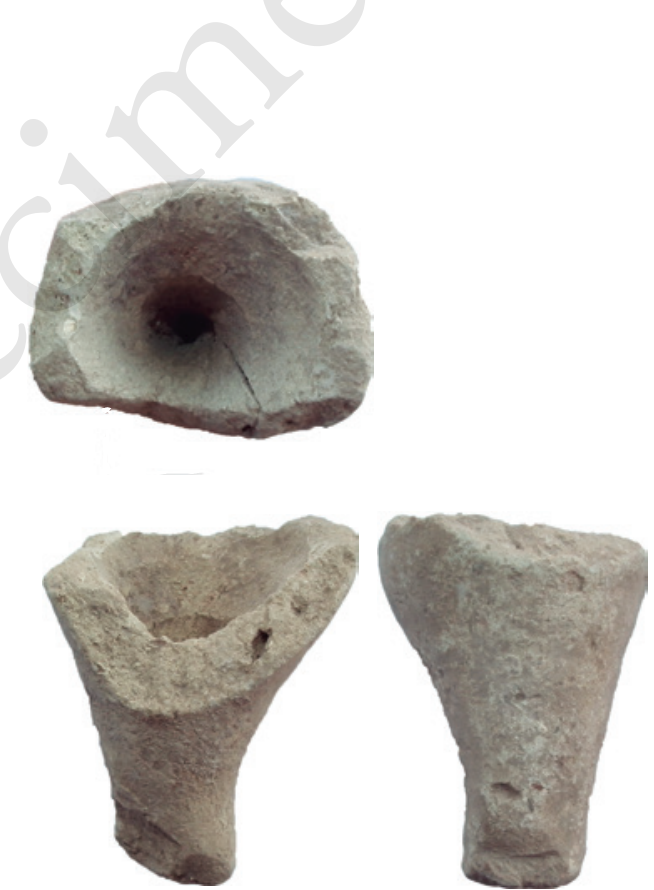

Med15-157

Marl A3

$\begin{array}{ll}0 & 1\end{array}$ $5 \mathrm{~cm}$

PL. 2. Céramique Moyen Empire. 


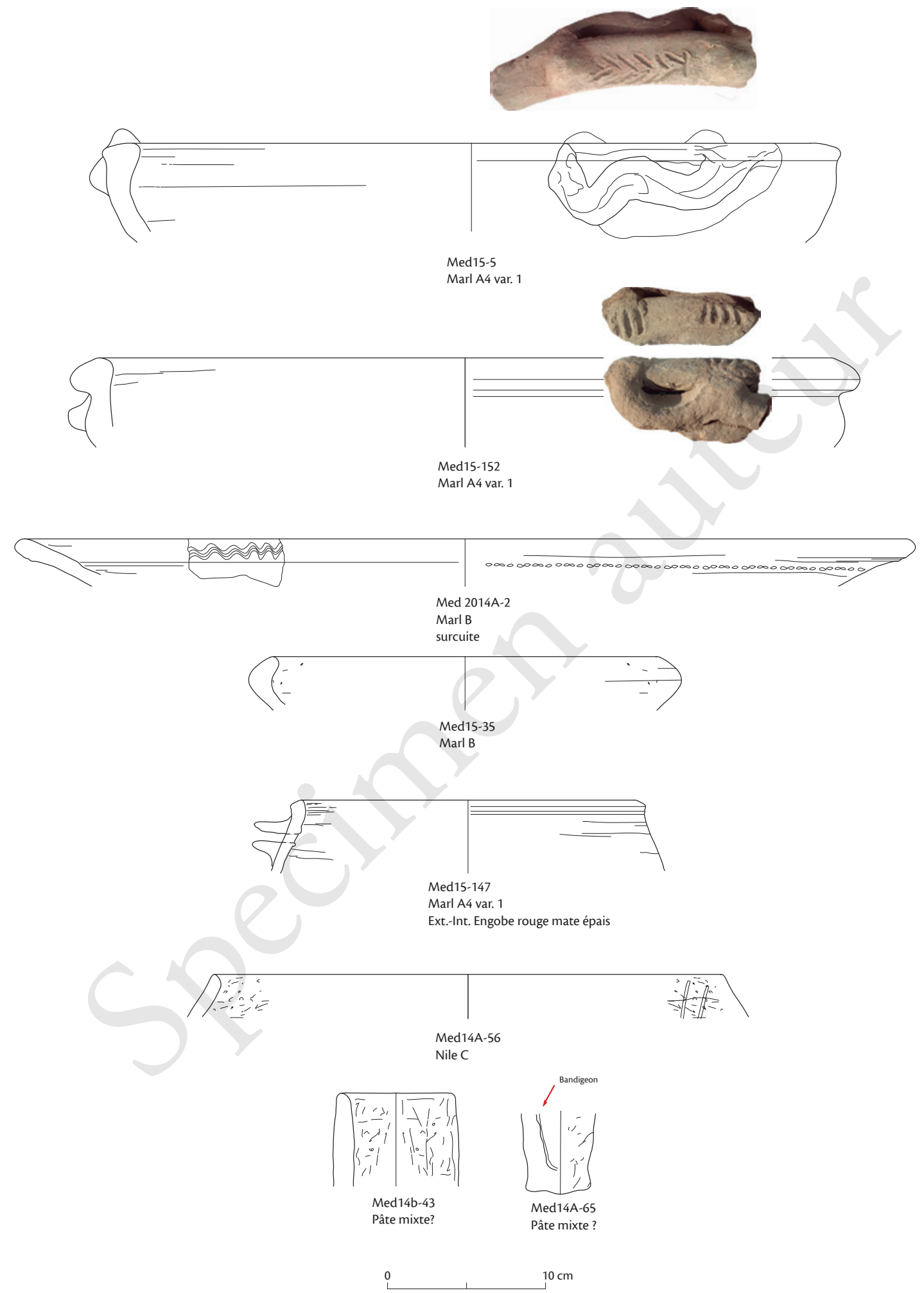

PL. 3. Céramique Deuxième Période intermédiaire. 


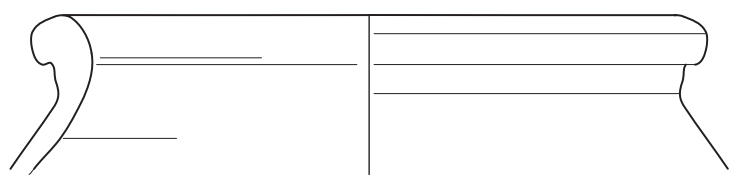

Med 2015-120

Marl A4 var. 1

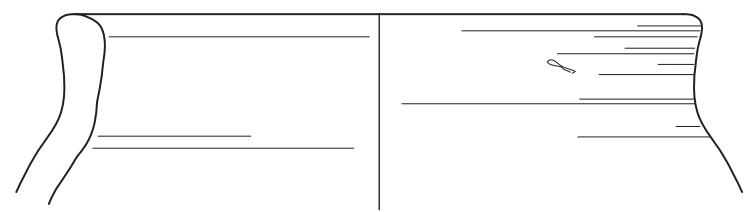

Med 2015-162

Marl A surcuite

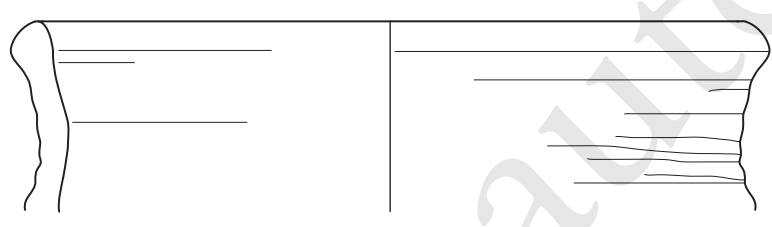

Med15-159

Marl A3
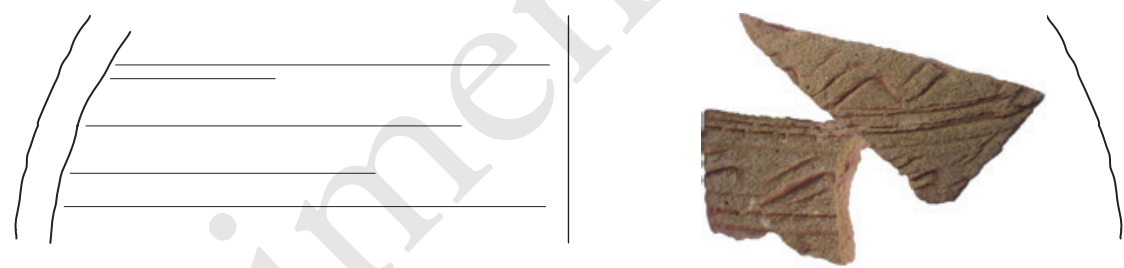

Med14A-26

Marl B surcuite

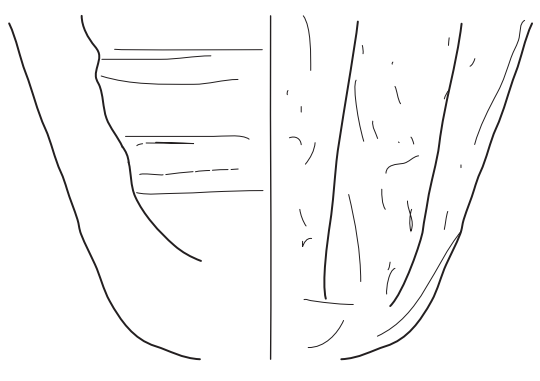

Med14A-21

Marl A4 surcuite

Ext.-Int. Beige

0

$10 \mathrm{~cm}$

Pl. 4. Céramique Deuxième Période intermédiaire. 


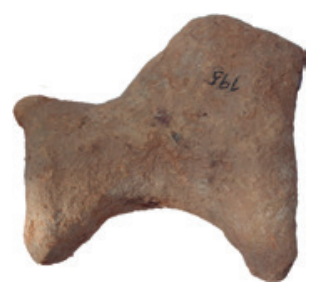

Med15-195

Marl A4

Figurine de cheval
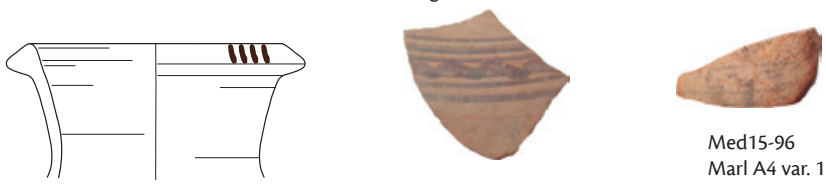

Med15-96

Marl A4 var. 1

Med15-K9-1

Marl A2

Med15-210

Marl A2

E
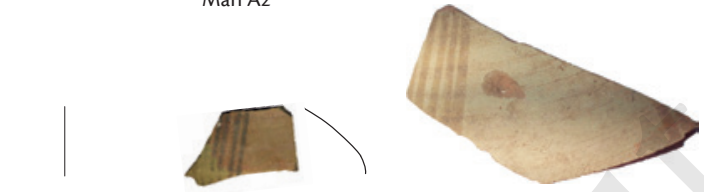

Med14A-80

Marl A2

Med15-173

Surcuite et déformée

Marl A2

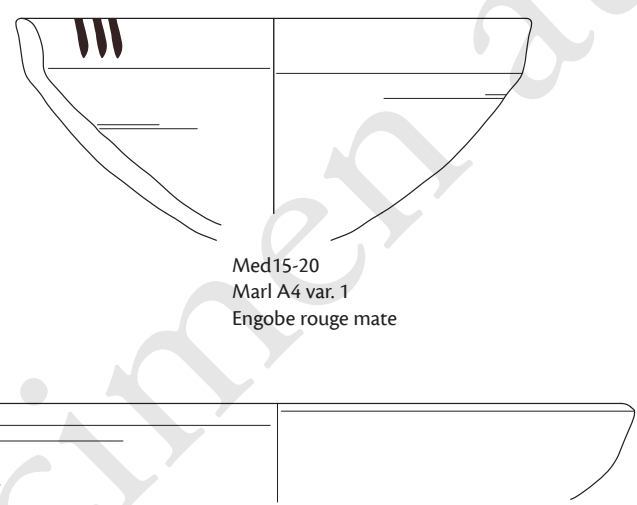

Med 2015-SP11-8

Marl A4 var. 1

Engobe rouge poli

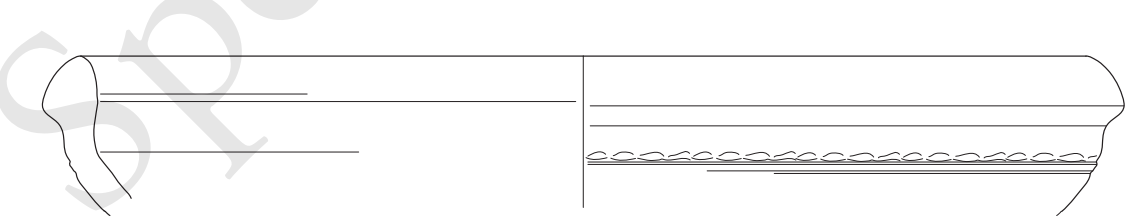

Med15-74

Marl A4 var. 1

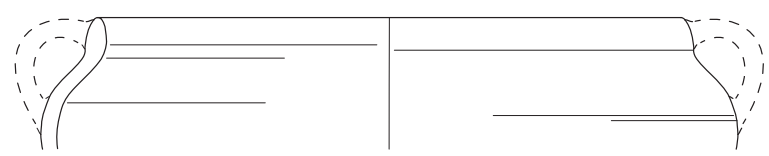

Med15-165

Marl A

Surcuite et déformée

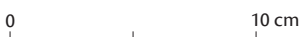

PL. 5. Céramique de la XVIII e dynastie. 

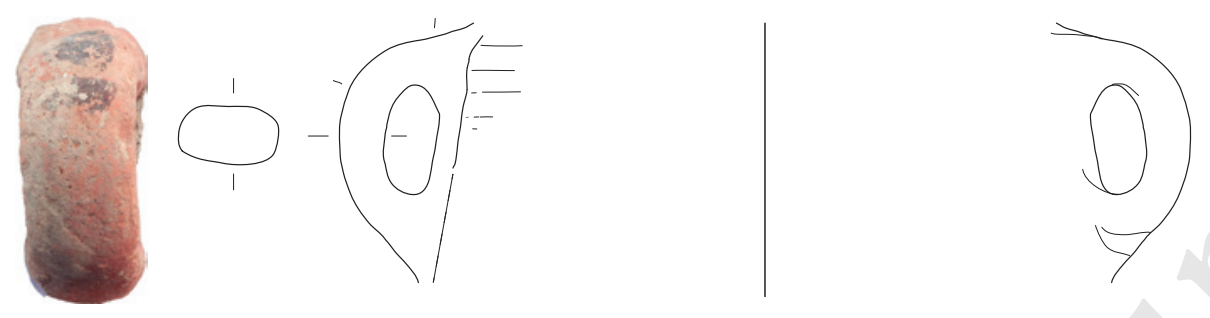

Med15-1

Marl A4 var. 1

Engobe rouge mate

Décoration peinte marron sur l'anse

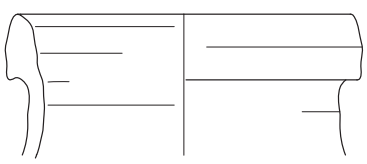

Med 2015-135

Marl A-4

Surcuite

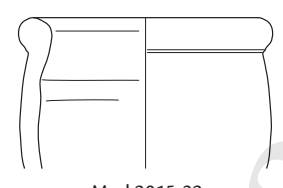

Med 2015-32

Marl A-4

Surcuite

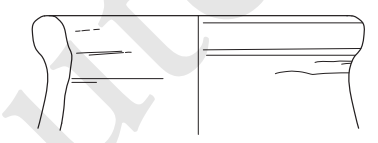

Med 2015-18

Marl A4 var. 1
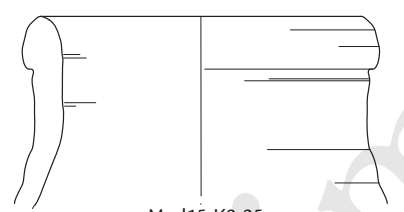

Med15-K9-25

Marl A

Surcuite

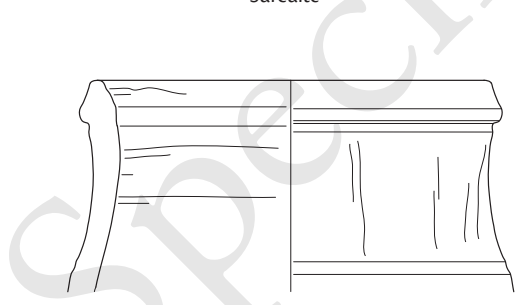

ed 2015-15

Marl A4 var. 1

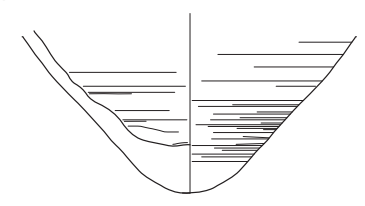

Med15-4

Marl A4 var. 1

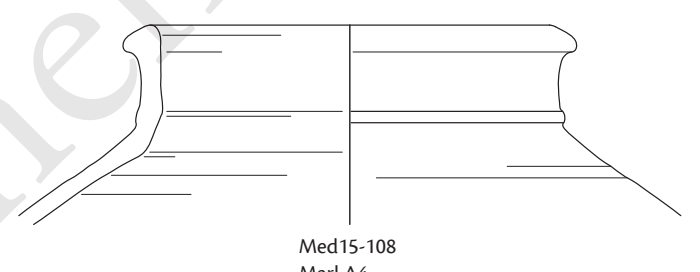

Marl A4

Surcuite et déformée

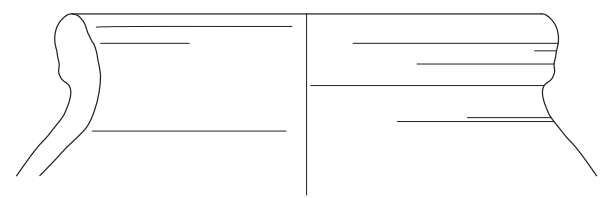

Med15-79

Marl A4 var. 1

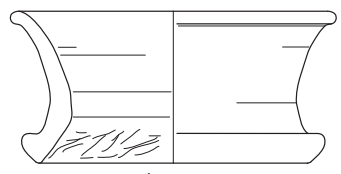

Med 2014A-51

Marl A4 var. 1

$\stackrel{0}{1} \quad 10 \mathrm{~cm}$

Pl. 6. Céramique de la XVIII ${ }^{e}$ dynastie. 


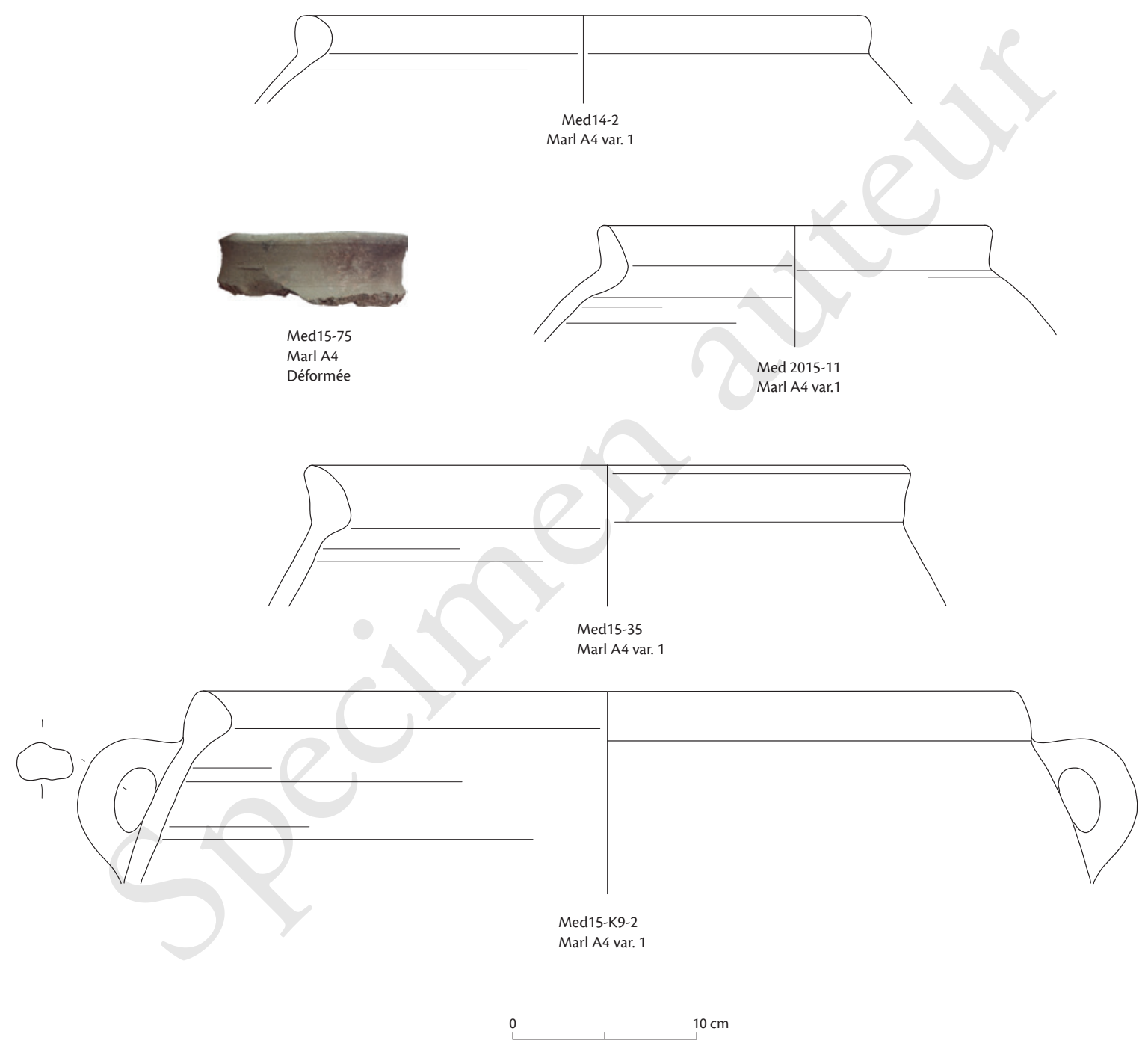

PL. 7. Céramique de l'époque ramesside. 


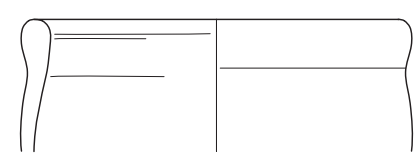

Med15-189

Marl A

Surcuite

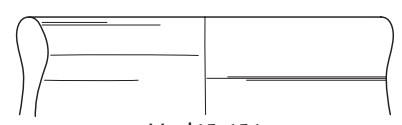

Med15-151

Marl A4 var. 1

Surcuite

Légèrement déformée

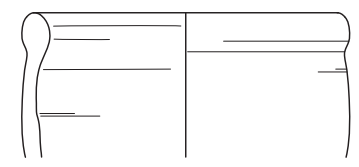

Med15-84

Marl A4 var. 1

Surcuite

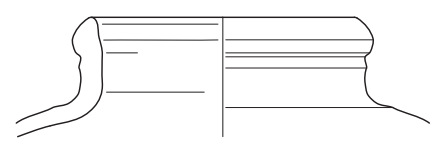

Med 2015-31

Marl A

Surcuite

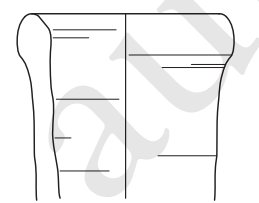

Med15-201

Marl A4

Surcuite

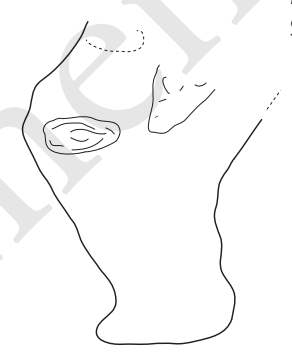

Med15-6

Marl A4 grossière

«Fire dog»
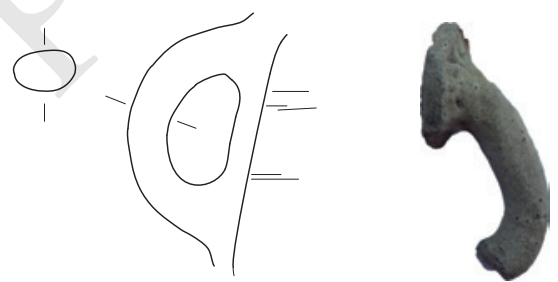

Med15-2

Marl A

Med15-93

Surcuite

Marl A

Surcuite

0 $10 \mathrm{~cm}$

PL. 8. Céramique de l'époque ramesside. 


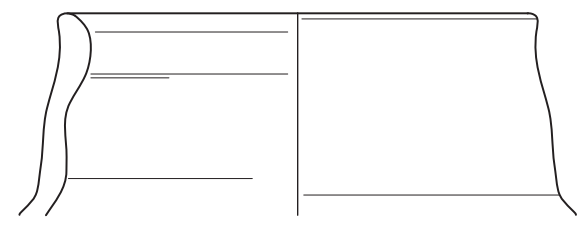

Med15-148

Marl A4 var. 1

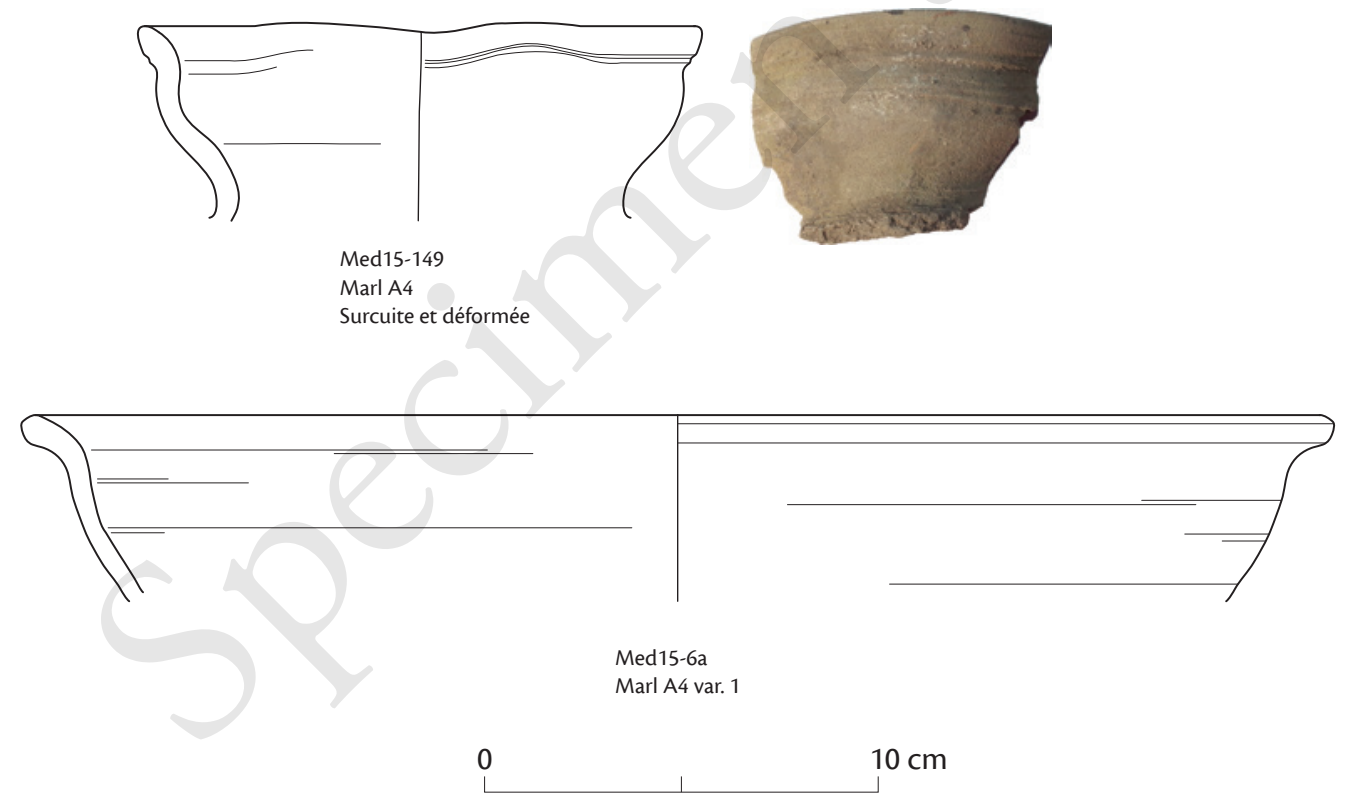

Pl. 9. Céramique de la Première Période intermédaire. 

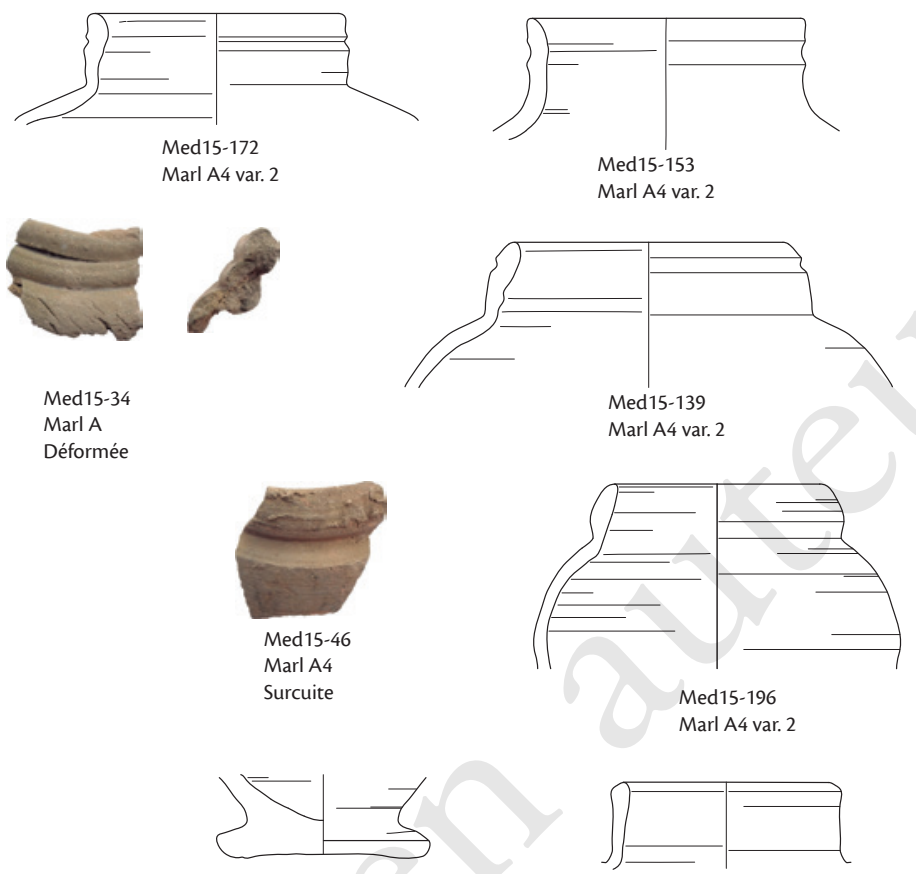

Med14A-101

Marl A4

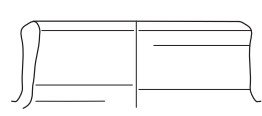

Grossière

Med15-22a

Marl A4 var. 2

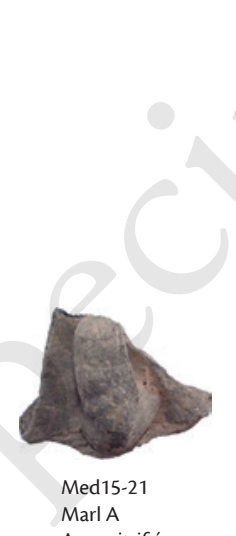

Marl A

Anse vitrifiée

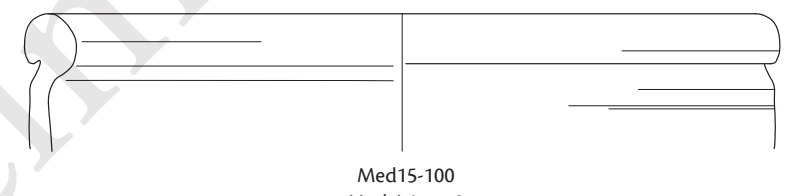

Marl A4 var. 2

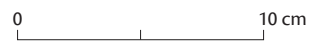

PL. ı. Céramique de la Basse Époque. 


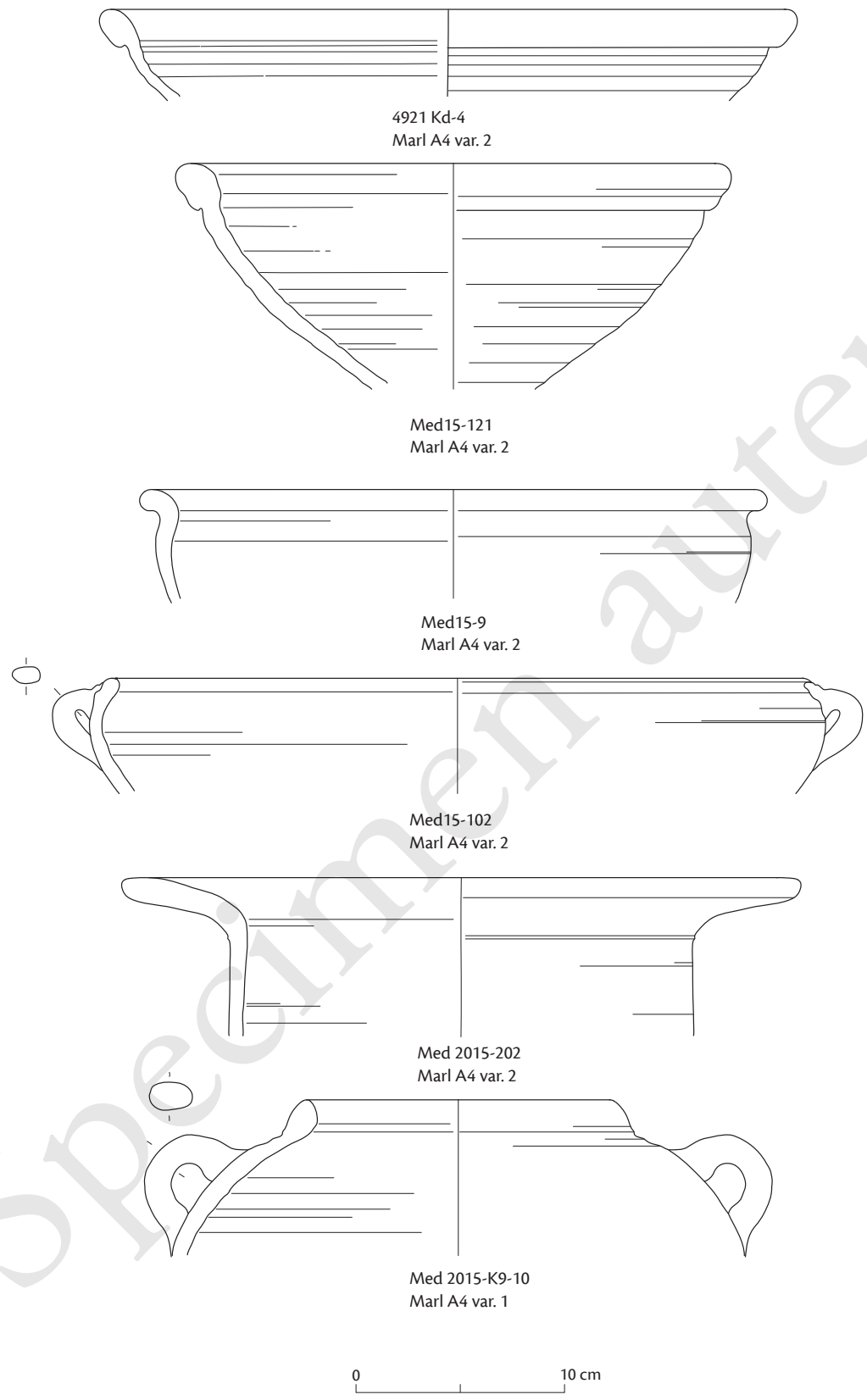

PL. II. Céramique de la Basse Époque. 

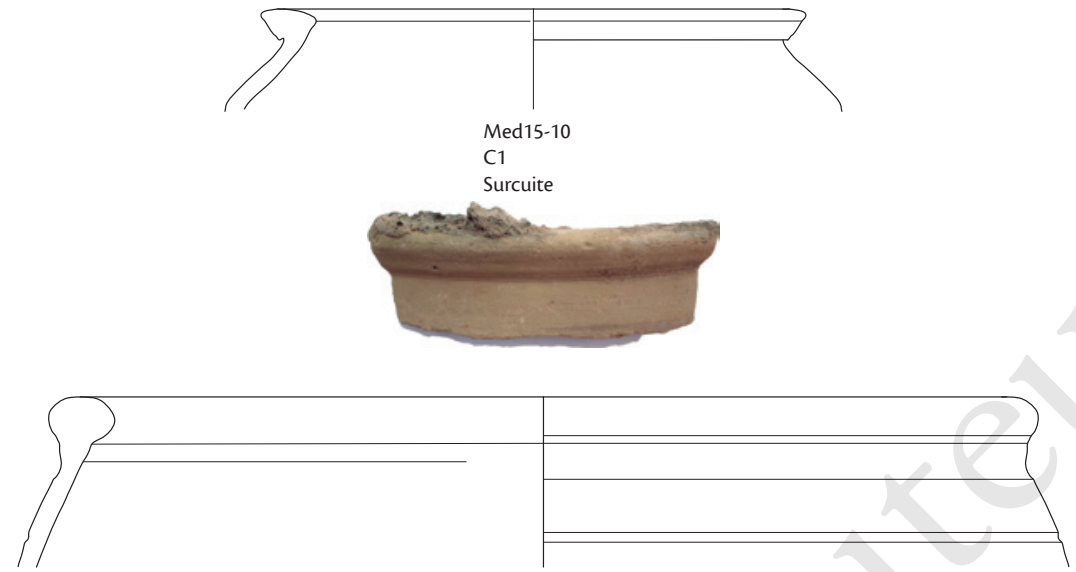

Med14B-3

C1

Surcuite

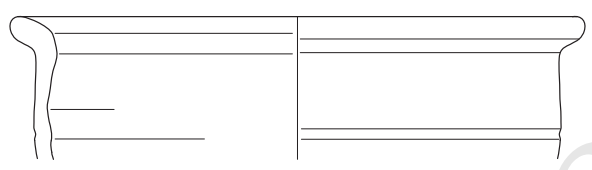

Med 2015-G7-33

C1

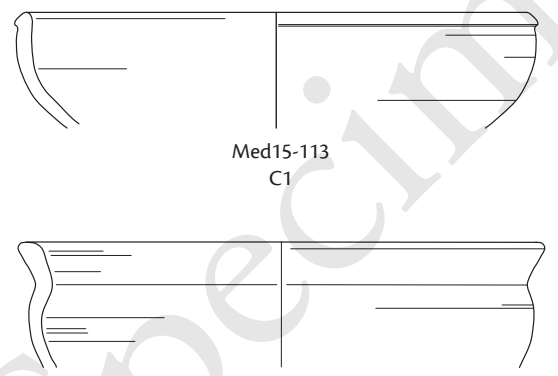

Med 2015-6

C1

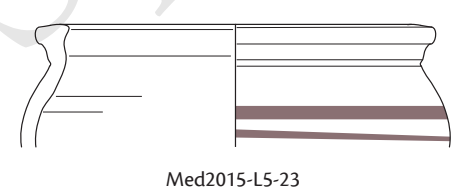

C1, Décoration peinte mauve

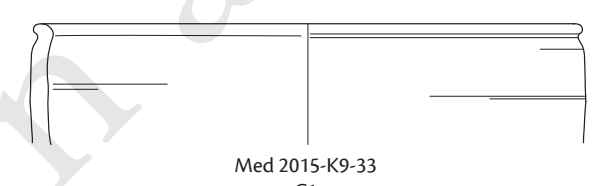

C1

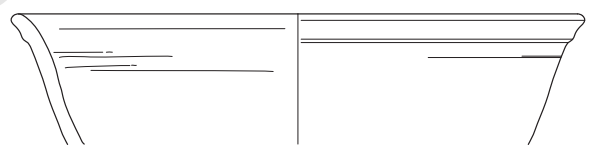

Med15-44

C1

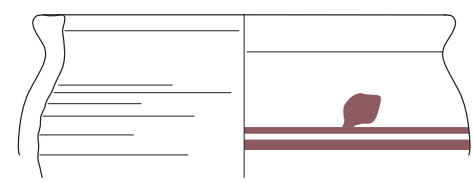

Med 2015-65

C1, engobe orange

Décoration peinte mauve

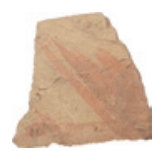

Med15-209

C1 Décoration peinte en rouge

0 $10 \mathrm{~cm}$

PL. I2. Céramique ptolémaïque. 


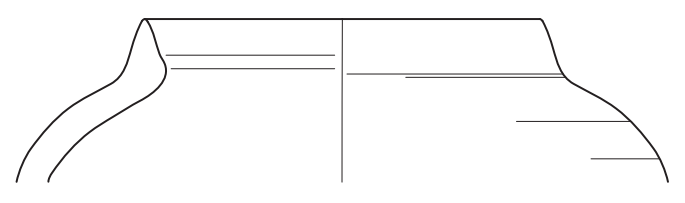

Med15-K9-24

Marl A

vitrifiée

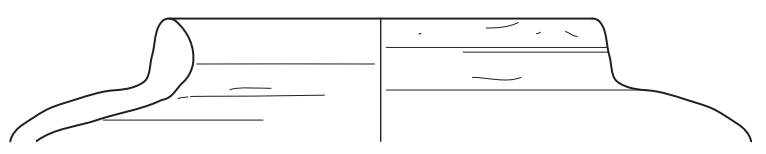

Med15-186

Marl A4

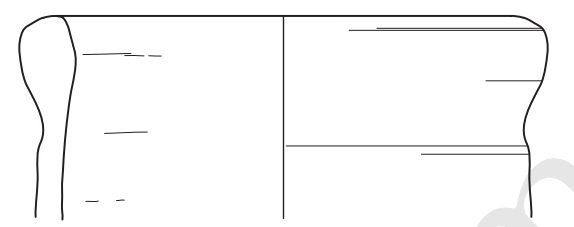

Med15-7

C3

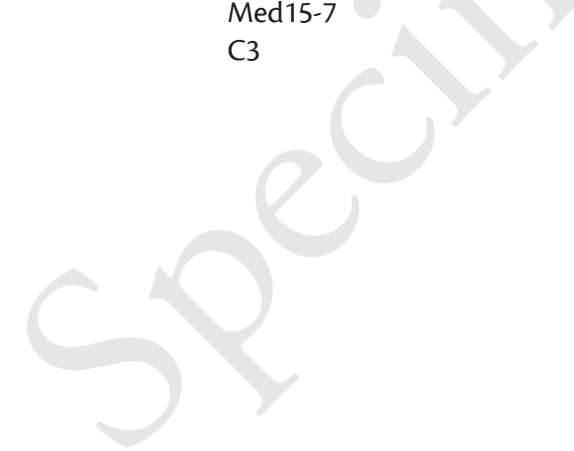

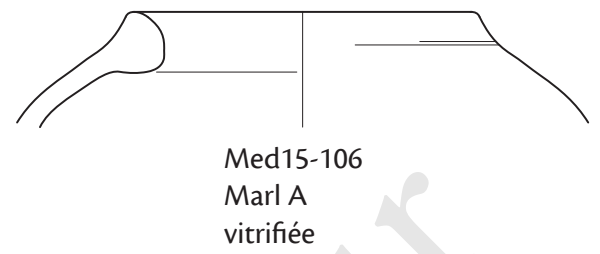

vitrifiée

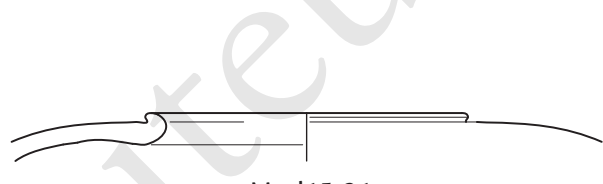

Med15-21

C1

vitrifiée

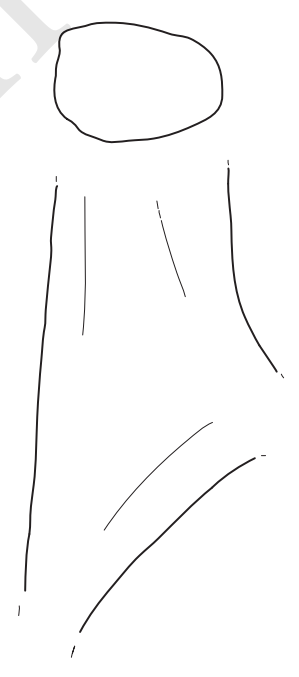

Med15-1b

C3 


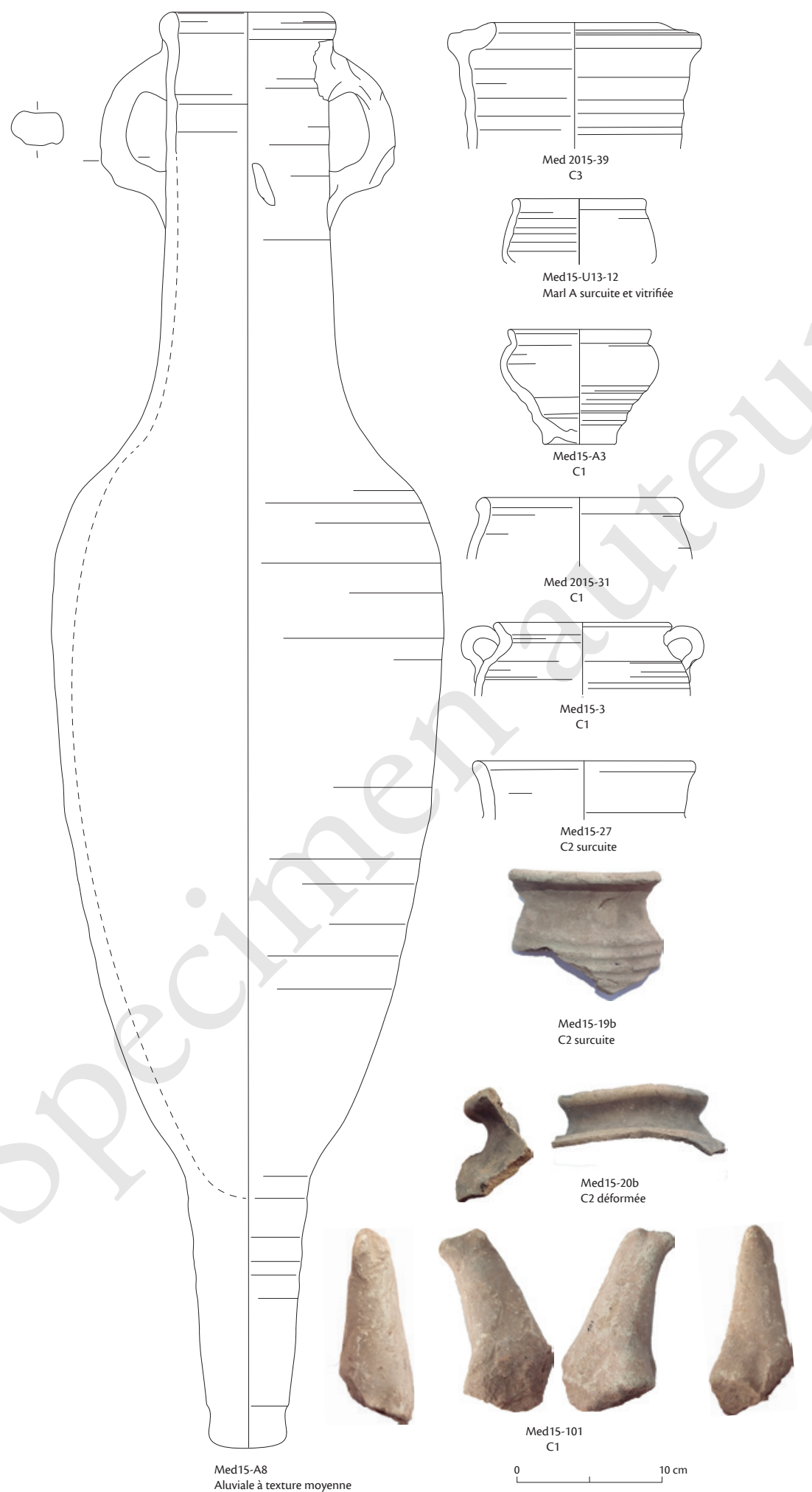

PL. I4. Céramique romaine. 

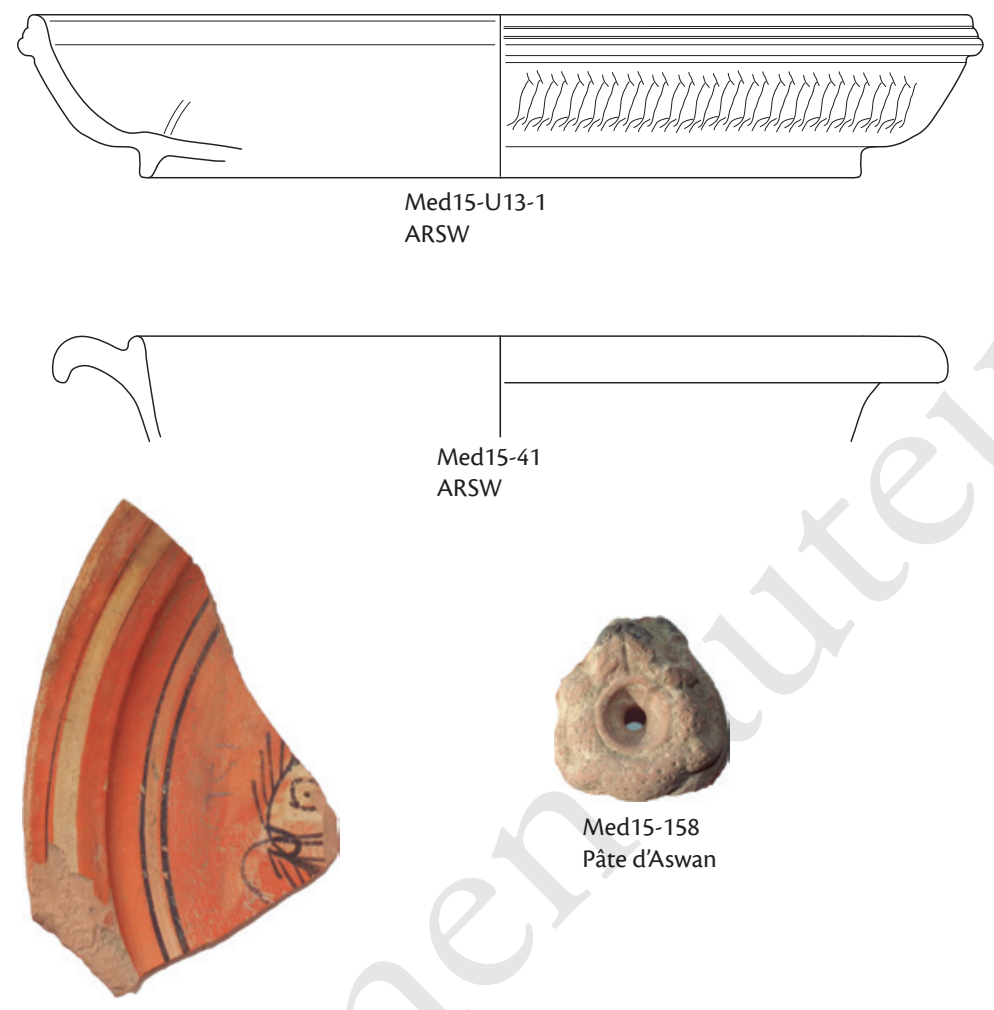

ARSW

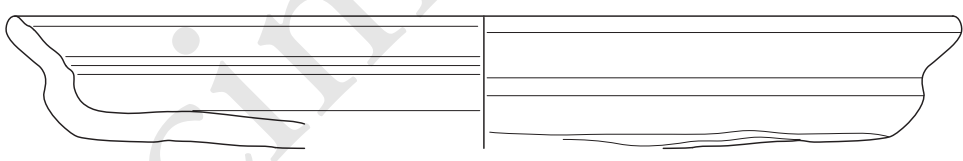

5581-11 M4264

Pâte d'Aswan

Engobe orange, décor peint
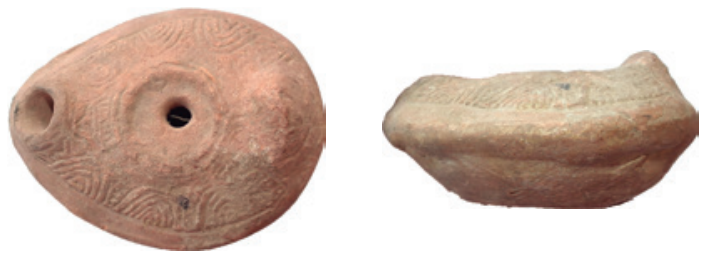

Med15-U13-4

Pàte d'Aswan

Med15-158

Pâte d'Aswan

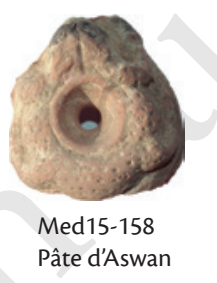




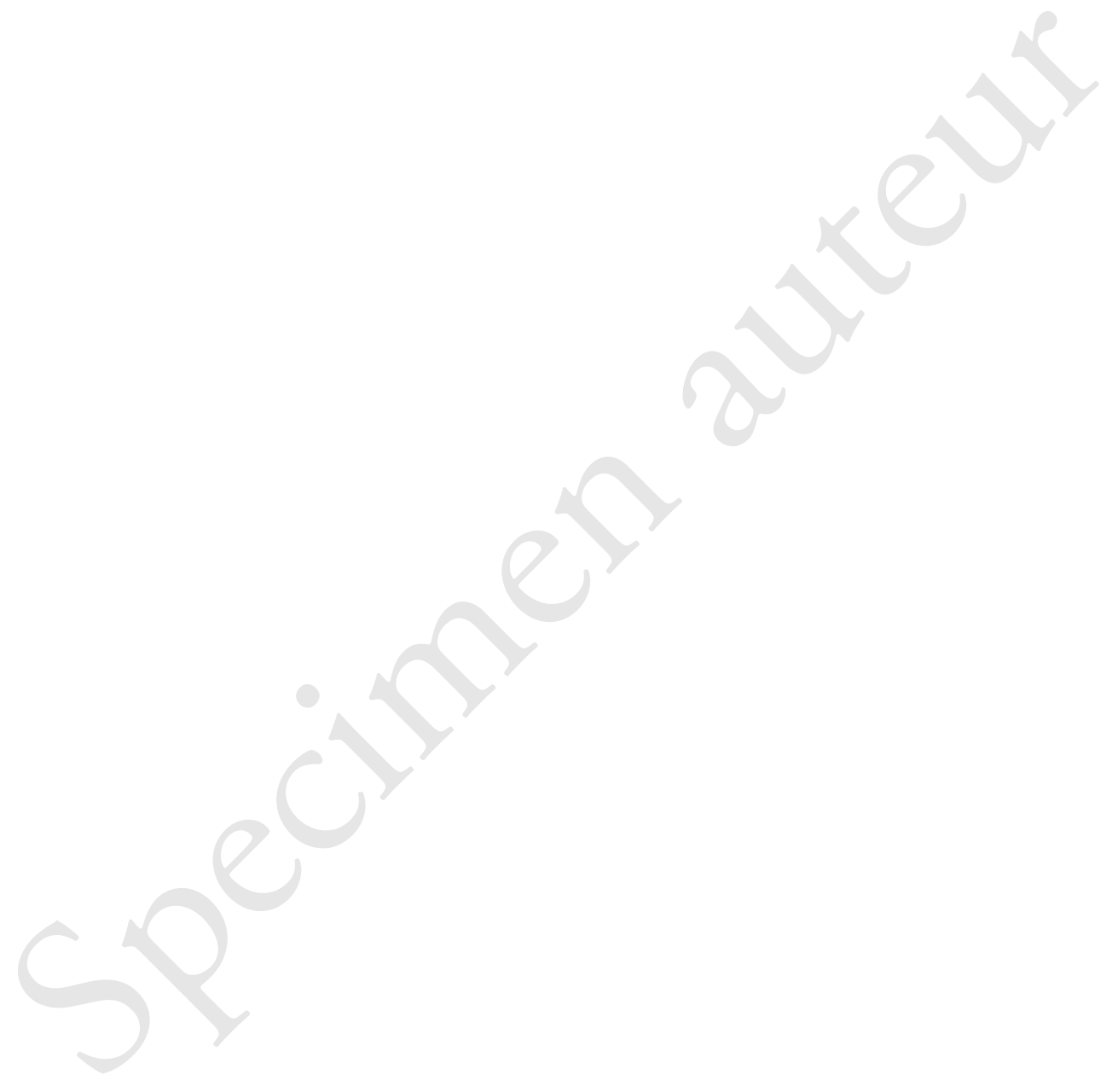

